\title{
Integer and Fractional Packings in Dense 3-Uniform Hypergraphs
}

\author{
Penny Haxell, ${ }^{1, *, \dagger}$ Brendan Nagle, ${ }^{2}$ Vojtěch Rödl ${ }^{3,}+$ \\ ${ }^{1}$ Department of Combinatorics and Optimization, University of Waterloo, \\ Waterloo, Ontario, Canada N2L 3G1; e-mail: pehaxell@math.uwaterloo.ca \\ ${ }^{2}$ School of Mathematics, Georgia Institute of Technology, Atlanta, Georgia 30332; \\ e-mail: nagle@math.gatech.edu \\ ${ }^{3}$ Department of Mathematics and Computer Science, Emory University, Atlanta, \\ Georgia 30322; e-mail: rodl@mathcs.emory.edu
}

Received 10 July 2000; accepted 30 January 2002

DOI 10.1002/rsa.10075

\begin{abstract}
Let $\mathscr{F}_{0}$ be any fixed 3-uniform hypergraph. For a 3-uniform hypergraph $\mathscr{H}$ we define $\nu_{\mathscr{F}_{0}}(\mathscr{H})$ to be the maximum size of a set of pairwise triple-disjoint copies of $\mathscr{F}_{0}$ in $\mathscr{H}$. We say a function $\psi$ from the set of copies of $\mathscr{F}_{0}$ in $\mathscr{H}$ to $[0,1]$ is a fractional $\mathscr{F}_{0}$-packing of $\mathscr{H}$ if $\sum_{\mathscr{F} \ni e} \psi(\mathscr{F}) \leq 1$ for every triple $e$ of $\mathscr{H}$. Then $\nu_{\mathscr{F}_{0}}^{*}(\mathscr{H})$ is defined to be the maximum value of

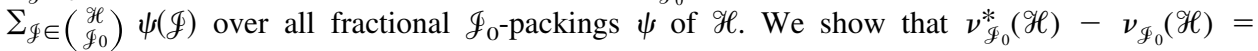
$o\left(|V(\mathscr{H})|^{3}\right)$ for all 3-uniform hypergraphs $\mathscr{H}$. This extends the analogous result for graphs, proved by Haxell and Rödl (2001), and requires a significant amount of new theory about regularity of 3-uniform hypergraphs. In particular, we prove a result that we call the Extension Theorem. This states that if a $k$-partite 3-uniform hypergraph is regular [in the sense of the hypergraph regularity lemma of Frankl and Rödl (2002)], then almost every triple is in about the same number of copies of $K_{k}^{(3)}$ (the complete 3-uniform hypergraph with $k$ vertices). (c) 2003 Wiley Periodicals, Inc. Random Struct. Alg., 22: 248-310, 2003
\end{abstract}

Keywords: integer and fractional packings; regularity lemma for hypergraphs

\footnotetext{
*Correspondence to: P. Haxell

$\dagger$ Partially supported by NSERC.

$\ddagger$ Partially supported by NSF Grant 9704114 .

(C) 2003 Wiley Periodicals, Inc.
} 


\section{INTRODUCTION}

Let $\mathscr{F}_{0}$ be a fixed 3-uniform hypergraph. For a 3-uniform hypergraph $\mathscr{H}$, we define the (integer) packing number $\nu_{\mathscr{F}_{0}}(\mathscr{H})$ to be the maximum size of a set of pairwise tripledisjoint copies of $\mathscr{F}_{0}$ in $\mathscr{H}$. A related parameter is the fractional packing number $\nu_{\mathscr{F}_{0}}^{*}(\mathscr{H})$, which is defined as follows. We denote by $\left(\begin{array}{l}\mathscr{H} \\ \mathscr{F}_{0}\end{array}\right)$ the set of all subhypergraphs of $\mathscr{H}$ that are isomorphic to $\mathscr{F}_{0}$. A fractional $\mathscr{F}_{0}$-packing of $\mathscr{H}$ is a function $\psi:\left(\begin{array}{l}\mathscr{H} \\ \mathscr{F}_{0}\end{array}\right) \rightarrow[0,1]$ with the property that $\Sigma_{\mathscr{F} \ni e} \psi(\mathscr{F}) \leq 1$ for every triple $e$ of $\mathscr{H}$. The value of a fractional $\mathscr{F}_{0}$-packing $\psi$ is defined to be $|\psi|=\sum_{\mathscr{F} \in\left(\mathscr{F}_{0}\right)}(\mathscr{\mathscr { F }})$. The parameter $\nu_{\mathscr{F}_{0}}^{*}(\mathscr{H})$ is then defined to be $\nu_{\mathscr{F}_{0}}^{*}(\mathscr{H})=\max \{|\psi|: \psi$ is a fractional packing of $\mathscr{H}\}$. Note then that any set $\mathscr{S}$ of pairwise triple-disjoint copies of $\mathscr{F}_{0}$ in $\mathscr{H}$ gives a fractional $\mathscr{F}_{0}$-packing $\psi_{0}$ of $\mathscr{H}$ with $\left|\psi_{0}\right|=|\mathscr{S}|$ such that $\psi_{0}(\mathscr{F}) \in\{0,1\}$ for every $\mathscr{E} \in\left(\begin{array}{c}\mathscr{H} \\ \mathscr{F}_{0}\end{array}\right)$. This implies that $\nu_{\mathscr{F}_{0}}(\mathscr{H}) \leq \nu_{\mathscr{F}_{0}}^{*}(\mathscr{H})$ for every $\mathscr{H}$.

The aim of this paper is to show that for large dense hypergraphs $\mathscr{H}$, the parameter $\nu_{\mathscr{F}_{0}}^{*}(\mathscr{H})$ gives a close approximation to $\nu_{\mathscr{F}_{0}}(\mathscr{H})$.

Theorem 1.1. Let $\mathscr{F}_{0}$ be a 3-uniform hypergraph and let $\eta>0$ be a real number. Then there exists $N_{1}=N_{1}\left(\mathscr{\Phi}_{0}, \eta\right)$ such that for every 3-uniform hypergraph $\mathscr{H}$ with $n \geq N_{1}$ vertices we have

$$
\nu_{\mathscr{F}_{0}}^{*}(\mathscr{H})-\nu_{\mathscr{F}_{0}}(\mathscr{H})<\eta n^{3} .
$$

Calculating $\nu_{\mathscr{F}_{0}}^{*}(\mathscr{H})$ is a linear programming problem, and hence can be solved in polynomial time. However the problem of calculating $\nu_{\mathscr{F}_{0}}(\mathcal{H})$ is NP-hard, in fact this is true even for the analogous problem in graphs. It was proved by Dor and Tarsi in [3] that, for any graph $H_{0}$ that contains a connected component with at least three edges, the problem of finding the maximum size of a set of pairwise edge-disjoint copies of $H_{0}$ in a general graph $G$ is NP-hard. Therefore, Theorem 1.1 gives an efficient algorithm for approximating $\nu_{\mathscr{F}_{0}}(\mathscr{H})$ for those hypergraphs $\mathscr{H}$ for which $\nu_{\mathscr{F}_{0}}(\mathscr{H})=\Omega\left(|V(G)|^{3}\right)$. Thus the problem of finding $\nu_{\mathscr{F}_{0}}(\mathscr{H})$ is another example of an NP-hard problem which has a polynomial time approximation algorithm for an appropriately defined "dense case." Various other such problems have been identified and studied by, e.g., Frieze and Kannan [7, 8] and Arora, Karger, and Karpinski [1] (see also [4] and [2]). The analogous result to Theorem 1.1 for graphs was proved in [9]. In many of these problems, including [9], the Regularity Lemma of Szemerédi [14] plays an important role.

The proof of Theorem 1.1 depends on a generalization of Szemerédi's Lemma to hypergraphs, which is due to Frankl and Rödl [6] (see Section 2). Our plan for proving that $\nu_{\mathscr{F}_{0}}(\mathscr{H})$ is closely approximated by $\nu_{\mathscr{F}_{0}}^{*}(\mathscr{H})$ for a given hypergraph $\mathscr{H}$ will be as follows. Using the hypergraph regularity lemma, we shall construct a special (finite) object called an $\ell$-augmented weighted 3-graph $\mathscr{A}$ from $\mathscr{H}$, for which there is a natural definition of $\nu_{\mathscr{F}_{0}}^{*}(\mathscr{A})$, and then show that both $\nu_{\mathscr{F}_{0}}(\mathscr{H})$ and $\nu_{\mathscr{F}_{0}}^{*}(\mathscr{H})$ are very close to a certain multiple of $\nu_{\mathcal{F}_{0}}^{*}(\mathscr{A})$. A major step in this proof will be to show that if a $k$-partite 3-uniform hypergraph is "regular," in a very specific sense required by the regularity lemma, then almost every triple is in about the same number of copies of $K_{k}^{(3)}$, the complete 3-uniform hypergraph with $k$ vertices. We refer to this result as the Extension Theorem. To establish this requires a foundation of structural theorems for hypergraphs that result from the use of the hypergraph regularity lemma. This work may be of independent interest, and could be useful for other problems in hypergraphs. We mention that the theory that would be 
required to extend our arguments to $t$-uniform graphs for any $t \geq 4$ is not currently available.

This paper is organized as follows. In Section 2 we describe the regularity lemma for hypergraphs. Section 3 describes how to construct the finite object $\mathscr{A}$ from a given hypergraph $\mathcal{H}$. Section 3 also contains a sketch of the proof of Theorem 1.1, the detailed proof appears in Section 4. The next five sections contain proofs of some auxiliary lemmas, and finally the structural theory related to the hypergraph regularity lemma is given in Sections 10-13. This includes the precise statement of the Extension Theorem which appears as Theorem 10.12.

\section{THE REGULARITY LEMMA FOR HYPERGRAPHS}

In this section we describe the regularity lemma for 3-uniform hypergraphs, due to Frankl and Rödl [6]. In the original Regularity Lemma of Szemerédi for graphs, it was shown that the vertex set of any large enough graph $G$ can be partitioned into a bounded number of classes, such that the following holds: For almost every pair of vertex classes $V_{i}$ and $V_{j}$, the bipartite subgraph of $G$ induced by $V_{i}$ and $V_{j}$ is "very uniform," that is, its edges are distributed in much the same way as one would expect in a random bipartite graph. In the Hypergraph Regularity Lemma, corresponding to the vertex partition in Szemerédi's lemma, we again have a partition $V_{1} \cup \cdots \cup V_{t}$ of the vertex set $V$ of our hypergraph $\mathscr{H}$, but we also have a partition of the set of pairs of vertices $\left\{\left\{v_{i}, v_{j}\right\}: v_{i} \in V_{i}, v_{j} \in\right.$ $\left.V_{j}, i \neq j\right\}$ into "bipartite graphs" that are themselves very highly regular. [See the definition of $(\ell, t, \gamma, \epsilon)$-partition below.] This partition is chosen in such a way that for most choices of three of these bipartite graphs that form a tripartite graph (called a "triad"), the subhypergraph of $\mathscr{H}$ induced by this triad is "very uniform," i.e., behaves much like a random 3-partite 3-uniform hypergraph. Therefore, in the Hypergraph Regularity Lemma, it is these triads that take the place of the pairs of vertex classes in Szemerédi's lemma.

We begin with some introductory background definitions and notation.

Definition 2.1. Let $G$ be a bipartite graph with vertex classes $V$ and $W$, and let $X \subseteq V$ and $Y \subseteq W$ be two nonempty vertex subsets. Then the density of the pair $X, Y$ with respect to $G$, denoted $d_{G}(X, Y)$, is defined by

$$
d_{G}(X, Y)=\frac{|\{\{x, y\} \in G: x \in X, y \in Y\}|}{|X||Y|} .
$$

We say that the bipartite graph $G$ is $(\alpha, \epsilon)$-regular if $\alpha(1-\epsilon)<d_{G}(X, Y)<\alpha(1+\epsilon)$ for every pair of subsets $X \subseteq V$ and $Y \subseteq W$ with $|X|>\epsilon|V|$ and $|Y|>\epsilon|W|$.

Definition 2.2. Let $G$ be a $k$-partite graph with a fixed $k$-partition $\left(V_{1}, \ldots, V_{k}\right)$. We shall write $G=\bigcup_{1 \leq i<j \leq k} G^{i j}$, where $G^{i j}=G\left[V_{i}, V_{j}\right]=\left\{\left\{v_{i}, v_{j}\right\} \in G: v_{i} \in V_{i}, v_{j} \in V_{j}\right\}$. Let $\ell>0, \epsilon>0$ be given. We call $G$ an $(\ell, \epsilon, k)$-cylinder if each bipartite graph $G^{i j}, 1 \leq i<$ $j \leq k$, is $(1 / \ell, \epsilon)$-regular. By a $k$-partite cylinder we simply mean a k-partite graph with fixed k-partition. 
It will be useful for us to have a very precise estimate on the size of the set of triangles contained in an $(\ell, \epsilon, 3)$-cylinder $G$. For this purpose we have the following. We let $\mathcal{K}_{3}(G)=\{\{x, y, z\}:\{x, y, z\}$ is the vertex set of a triangle in $G\}$.

Fact 2.3. For any positive integer $\ell$ and positive real $\theta$, there exists $\epsilon_{1}=\epsilon_{1}(\ell, \theta)$ so that whenever $G$ is an $\left(\ell, \epsilon_{1}, 3\right)$-cylinder with vertex partition $\left(V_{1}, V_{2}, V_{3}\right)$, where $\left|V_{1}\right|=\left|V_{2}\right|$ $=\left|V_{3}\right|=n$, then

$$
(1-\theta) \frac{n^{3}}{\ell^{3}}<\left|\mathscr{K}_{3}(G)\right|<(1+\theta) \frac{n^{3}}{\ell^{3}} .
$$

The proof of this fact follows easily from the definition of an $(\ell, \epsilon, k)$-cylinder.

Definition 2.4. Let $V$ be a set. An $(\ell, t, \gamma, \epsilon)$-partition $\mathscr{P}$ of $V$ is an (auxiliary) partition $V=V_{0} \cup V_{1} \cup \cdots \cup V_{t}$ of $V$, together with a system of edge-disjoint bipartite graphs $\mathscr{B}$ $=\left\{P_{\beta}^{i j}: 1 \leq i<j \leq t, 0 \leq \beta \leq \ell_{i j} \leq \ell\right\}$, such that

(i) $\left|V_{0}\right|<t$ and $|V 1|=|V 2|=\cdots=|V t|=\lfloor n / t\rfloor \stackrel{\text { def }}{=} m$,

(ii) $\bigcup_{\beta=0}^{\ell_{i j}} P_{\beta}^{i j}=K\left(V_{i}, V_{j}\right)$ for all $i, j, 1 \leq i<j \leq t$.

(iii) For all but at most $\gamma\left(\begin{array}{c}t \\ 2\end{array}\right)$ pairs $i, j, 1 \leq i<j \leq t$, we have that $\left|P_{0}^{i j}\right| \leq \gamma m^{2}$ and $P_{\beta}^{i j}$ is $(1 / \ell$, $\epsilon)$-regular for all $\beta=1, \ldots, \ell_{i j}$.

Definition 2.5. If $\mathscr{P}$ is an $(\ell, t, \gamma, \epsilon)$-partition of $V$ and the bipartite graphs $P_{\beta}^{i j}, P_{\rho}^{i s}$, and $P_{\gamma}^{j s}$ are all $(1 / \ell, \epsilon)$-regular, then $a$ triad $P=\left(P_{\beta}^{i j}, P_{\rho}^{i s}, P_{\gamma}^{j s}\right)$ of $\mathscr{P}$ is the 3-partite graph with vertex set $V_{i} \cup V_{j} \cup V_{s}$ formed from the union of these three bipartite graphs.

Note that a triad of $\mathscr{P}$ is an $(\ell, \epsilon, 3)$-cylinder. In fact, more generally, it can be shown that if $k$ is fixed and $\gamma$ and $\epsilon$ are sufficiently small, then the $(\ell, t, \gamma, \epsilon)$-partition $\mathscr{P}$ has the following property: If we choose $k$ vertex classes of $\mathscr{P}$, and one bipartite graph $P_{\beta}^{i j}$ joining each pair of these classes, then the union of these bipartite graphs almost always forms an $(\ell, \epsilon, k)$-cylinder.

We now give definitions pertaining to 3-uniform hypergraphs.

Definition 2.6. We refer to any k-partite, 3-uniform hypergraph H with a fixed $k$ partition $\left(V_{1}, \ldots, V_{k}\right)$ as a $k$-partite 3 -cylinder. If $G$ is a k-partite cylinder with the same vertex partition, then we say $G$ underlies $\mathscr{H}$ if $\mathscr{H} \subseteq \mathscr{K}_{3}(G)$.

Usually we shall use this terminology in the following context: $\mathscr{H}$ is a 3-uniform hypergraph, $\mathscr{P}$ is an $(\ell, t, \gamma, \epsilon)$-partition of the vertex set $V$ of $\mathscr{H}$, and $G$ is an $(\ell, \epsilon$, $k$ )-cylinder formed by choosing $k$ vertex classes of $\mathscr{P}$, say $V_{i}, i \in K,|K|=k$, and one $P_{\beta_{i j}}^{i j}$ joining each pair $\left(V_{i}, V_{j}\right)$ of classes, where $i, j \in K, i \neq j$. Then each triad $P=\left(P_{\beta_{i j}}^{i j}\right.$, $\left.P_{\beta_{i s}}^{i s}, P_{\beta_{j s}}^{j s}\right)$ underlies a subhypergraph of $\mathcal{H}$, which we shall denote by $\mathscr{H}(P)$, consisting of all triples in $\mathcal{H}$ that are triangles in $P$. We call $\mathscr{H}(P)$ the 3-partite 3-cylinder of $\mathscr{H}$ on $P$.

The Hypergraph Regularity Lemma will basically say that the vertex set of any large enough 3-uniform hypergraph $\mathscr{H}$ has an $(\ell, t, \gamma, \epsilon)$-partition $\mathscr{P}$ such that most 3-partite 3 -cylinders $\mathscr{H}(P)$ of $\mathscr{H}$ will be "very regular." To make this concept precise, we need the following definitions. 
Definition 2.7. Let $\mathscr{H}$ be a 3-partite 3-cylinder with underlying 3-partite cylinder $G=$ $G^{12} \cup G^{23} \cup G^{13}$. We define the density $d_{\mathscr{H}}(G)$ of $\mathscr{H}$ with respect to $G$ as

$$
d_{\mathscr{H}}(G)= \begin{cases}\frac{\left|\mathscr{H} \cap \mathscr{K}_{3}(G)\right|}{\left|\mathscr{K}_{3}(G)\right|} & \text { if }\left|\mathscr{K}_{3}(G)\right|>0 \\ 0 & \text { otherwise. }\end{cases}
$$

In other words, the density counts the proportion of triangles of $G$ which are triples of $\mathcal{H}$.

More generally, let $Q \subseteq G$, where $Q=Q^{12} \cup Q^{13} \cup Q^{23}$ and $Q^{i j} \subseteq G^{i j}$. One can define the density $d_{\mathscr{H}}(Q)$ of $\mathscr{H}$ with respect to $Q$ as

$$
d_{\mathscr{H}}(Q)= \begin{cases}\frac{\left|\mathscr{H} \cap \mathscr{K}_{3}(Q)\right|}{\left|\mathscr{K}_{3}(Q)\right|} & \text { if }\left|\mathscr{K}_{3}(Q)\right|>0 \\ 0 & \text { otherwise. }\end{cases}
$$

A natural definition of regularity for a 3-partite 3 -cylinder $\mathscr{H}$ would be that for every $Q$ as above that contains a positive proportion of $\mathscr{K}_{3}(G)$, the density of $\mathscr{H}$ with respect to $Q$ is about the same. However it turns out that in many applications this is not a strong enough notion. We therefore introduce a concept called $(\delta, r)$-regularity. Here we use the notation $[a]=\{1, \ldots, a\}$, and by $[a]^{t}$ we mean $\{S \subseteq[a]:|S|=t\}$.

Definition 2.8. Let $\mathcal{H}$ be a 3-partite 3-cylinder with underlying 3-partite cylinder $G=$ $G^{12} \cup G^{23} \cup G^{13}$. Let $\vec{Q}=(Q(1), \ldots, Q(r))$ be an r-tuple of 3-partite cylinders $Q(s)=\bigcup_{\{i, j\} \in[3]^{2}} Q^{i j}(s)$ satisfying that for every $s \in[r],\{i, j\} \in[3]^{2}, Q^{i j}(s) \subseteq G^{i j}$.We define the density $d_{\mathcal{H}}(\vec{Q})$ of $\vec{Q}$ as

$$
d_{\mathscr{H}}(\vec{Q})= \begin{cases}\frac{\left|\mathscr{H} \cap \bigcup_{s=1}^{r} \mathscr{K}_{3}(Q(s))\right|}{\left|\bigcup_{s=1}^{r} \mathscr{K}_{3}(Q(s))\right|} & \text { if }\left|\bigcup_{s=1}^{r} \mathscr{K}_{3}(Q(s))\right|>0 \\ 0 & \text { otherwise. }\end{cases}
$$

Let a positive integer $r$ and a real $\delta>0$ be given. We say that the 3-cylinder $\mathscr{H}$ is $(\alpha$, $\delta, r)$-regular with respect to $G$ if for any $r$-tuple of 3-partite cylinders $\vec{Q}=(Q(1), \ldots$, $Q(r))$ as above, if

$$
\left|\bigcup_{s=1}^{r} \mathscr{K}_{3}(Q(s))\right|>\delta\left|\mathscr{K}_{3}(G)\right|,
$$

then

$$
\left|d_{\mathscr{H}}(\vec{Q})-\alpha\right|<\delta
$$

We say $\mathscr{H}$ is $(\delta, r)$-regular with respect to $G$ if it is $(\alpha, \delta, r)$-regular for some $\alpha$, and refer to $\alpha$ as the density of $\mathcal{H}$. If the regularity condition fails to be satisfied for any $\alpha$, we say that $\mathscr{H}$ is $(\delta, r)$-irregular with respect to $G$. 
Note that if $\mathscr{H}$ is $(\delta, r)$-regular with respect to $G$ and $\delta^{\prime} \geq \delta$ and $r^{\prime} \leq r$ is an integer, then $\mathscr{H}$ is also $\left(\delta^{\prime}, r^{\prime}\right)$-regular with respect to $G$.

Definition 2.9. Let $\mathscr{H}$ be a 3-uniform hypergraph with vertex set $V$, where $|V|=n$. We say that an $(\ell, t, \gamma, \epsilon)$-partition $\mathscr{P}$ of $V$ is $(\delta, r)$-regular for $\mathscr{H}$ if

$$
\sum\left\{\left|\mathscr{K}_{3}(P)\right|: P \text { is a triad of } \mathscr{P}, \mathscr{H}(P) \text { is }(\delta, r) \text {-irregular w.r.t. } P\right\}<\delta n^{3} \text {. }
$$

This implies in particular that if $\mathscr{H}$ is a dense hypergraph, then most of the triples of $\mathscr{H}$ belong to $(\delta, r)$-regular triads of the partition $\mathscr{P}$. We now state the Regularity Lemma of [6].

Theorem 2.10. For every $\delta$ and $\gamma$ with $0<\gamma \leq 2 \delta^{4}$, for all integers $t_{0}$ and $\ell_{0}$ and for all integer-valued functions $r(t, \ell)$ and all functions $\epsilon(\ell)>0$, there exist $T_{0}, L_{0}$, and $N_{0}$ such that any 3-uniform hypergraph $\mathscr{H} \subseteq[n]^{3}, n \geq N_{0}$, admits a $(\delta, r(t, \ell))$-regular, $(\ell$, $t, \gamma, \epsilon(\ell)$ )-partition for some $t$ and $\ell$ satisfying $t_{0} \leq t<T_{0}$ and $\ell_{0} \leq \ell<L_{0}$.

We shall apply a slight extension of this theorem. Let 2 be a partition of the vertex set of a 3 -uniform hypergraph $\mathscr{H}$. We say that the partition $\mathscr{P}$ respects the partition 2 if every vertex class of $\mathscr{P}$ is entirely contained in some vertex class of 2 . Then the following can be proved with a minor alteration of the proof of [6].

Theorem 2.11. For every $\delta$ and $\gamma$ with $0<\gamma \leq 2 \delta^{4}$, for all integers $q$, $t_{0}$, and $\ell_{0}$ and for all integer-valued functions $r(t, \ell)$ and all functions $\epsilon(\ell)>0$, there exist $T_{0}, L_{0}$, and $N_{0}$ such that the following holds. For any 3-uniform hypergraph $\mathcal{H} \subseteq[n]^{3}$ with $n \geq N_{0}$, and any partition 2 of the vertices of $\mathcal{H}$ into at most $q$ classes, there exists a $(\delta, r(t$, $\ell)$ )-regular, $(\ell, t, \gamma, \epsilon(\ell))$-partition of $\mathcal{H}$ that respects 2 , for some $t$ and $\ell$ satisfying $t_{0} \leq$ $t<T_{0}$ and $\ell_{0} \leq \ell<L_{0}$.

\section{THE CONSTRUCTION}

Before beginning the details of the construction of the finite object $\mathscr{A}$ mentioned in the introduction, we first outline the idea behind the proof of Theorem 1.1. A more detailed sketch of the proof will follow, and the full proof will be given in the next section. Our aim is to find a large family of triple-disjoint copies of $\mathscr{E}_{0}$ in $\mathscr{H}$, and the proof is based on the following fact (see Lemma 3.4). Given a fixed 3-uniform hypergraph $\mathscr{F}$ with vertex set [k], we consider a special type of $k$-partite 3 -cylinder $\mathscr{L}=\mathscr{L}(\mathscr{F})$ related to $\mathscr{E}$. Let the vertex set of $\mathscr{L}$ be $V_{1} \cup \cdots \cup V_{k}$, where the $V_{i}$ are disjoint sets of equal size, and let the underlying $k$-partite cylinder $G$ of $\mathscr{L}$ be an $(\ell, \epsilon, k)$-cylinder. For each triple $\{i, j, s\}$ of $\mathscr{J}$, let $\mathscr{L}$ have an $(\alpha, \delta, r)$-regular 3-partite 3-cylinder on the triad $\left(G^{i j}, G^{i s}, G^{j s}\right)$. We can think of $\mathscr{L}$ informally as a "blown-up copy of $\mathscr{E}$ of density $\alpha$." Then most triples in $\mathscr{L}$ are in about the same number $D$ of copies of $\mathscr{F}$, and for any pair of distinct triples, the number of copies of $\mathscr{F}$ that contain both is $o(D)$. Then there is a packing theorem due to Frankl and Rödl (actually we will apply a generalization due to Kahn) that says that in this situation, there exists a nearly perfect packing of copies of $\mathscr{E}$ in $\mathscr{L}$, that is, a $\mathscr{F}$-packing that covers almost every triple in $\mathscr{L}$. In other words, $\mathscr{L}$ has as large a $\mathscr{L}$-packing as is possible. 
(We remark that the structural theory that appears in Section 10 is required for the proof of this fact.)

Now $\mathcal{H}$ is an arbitrary hypergraph, so in general it will not at all resemble a blown-up copy of $\mathscr{E}_{0}$. However, the hypergraph regularity lemma will enable us to partition $\mathscr{H}$ into a large but finite number of triple-disjoint 3-partite 3-cylinders (while the size of $\mathscr{H}$ may tend to infinity), and then we will be able to reassemble these 3-partite 3-cylinders into many triple-disjoint blown-up copies $\mathscr{L}(\mathscr{F})$ of $\mathscr{F}_{0}$. Then, inside each $\mathscr{L}(\mathscr{E})$, we will find a nearly-perfect packing, and taking the union of all of these packings will result in a large packing of $\mathscr{H}$.

Many applications of Szemerédi's Regularity Lemma for graphs follow a rather standard format: We apply the lemma to our graph $H$ to obtain a finite weighted "cluster graph" $H_{0}$, which has one vertex for each vertex class of the regular partition, and one edge of weight $\alpha$ for every regular pair of vertex classes in $H$ of density $\alpha$. Then we find some particular substructure in $H_{0}$, and then prove that this corresponds to some desired substructure in $H$. Our approach here will be the same, but when we apply the Hypergraph Regularity Lemma to a hypergraph $\mathscr{H}$, we obtain a finite "cluster" object which is slightly more complicated than a weighted hypergraph. We call such an object an $\ell$-augmented weighted 3-graph.

Definition 3.1. An $\ell$-augmented weighted 3-graph $\mathscr{A}$ consists of

(i) a set $V$ of vertices,

(ii) a multiset $C=\bigcup_{v \neq w \in V}\left\{c_{1}^{v w}, \ldots c_{\ell_{v w} w}^{v w}\right.$ of pairs of vertices, where $c_{\rho}^{v w}$ joins $v$ and $w$ and $\ell_{v w} \leq \ell$

(iii) a set $E$ of triples, where each triple e consists of three pairs $c_{\rho}^{v w}, c_{\beta}^{v x}$, and $c_{\gamma}^{w x}$ (note then that each triple also determines three vertices $v, w$, and $x$ ),

(iv) a weight function $\omega: E \rightarrow[0,1]$.

Notice that a 3-uniform hypergraph can be thought of as a 1-augmented weighted 3 -graph in which every triple has weight 1.

Definition 3.2. Let $\mathscr{A}$ be an $\ell$-augmented weighted 3-graph, and let $\mathscr{F}_{0}$ be a hypergraph with vertex set $V\left(\mathscr{F}_{0}\right)=[k]$. Let $C\left(\mathscr{F}_{0}\right)=\left\{\{i, j\} \in\left(\begin{array}{c}V\left(\mathscr{F}_{0}\right) \\ 2\end{array}\right):\{i, j\} \in\right.$ e for some e $\left.\in \mathscr{F}_{0}\right\}$. Then a copy $\mathscr{F}$ of $\mathscr{F}_{0}$ in $\mathscr{A}$ consists of the following:

(i) a vertex set $V(\mathscr{F})=\left\{v_{1} \ldots, v_{k}\right\} \subset V(\mathscr{A})$,

(ii) a pair set $C(\mathscr{F})$ consisting of one pair $c_{\beta_{i, j}}^{v_{i} v_{j}} \in C(\mathscr{A})$ joining vertices $v_{i}$ and $v_{j}$ for each $\{i$, $j\} \in C\left(\mathscr{\Phi}_{0}\right)$,

(iii) a triple set $E(\mathscr{F})$, consisting of one triple of $\mathscr{A}$ with pairs $c_{\beta_{i, j}}^{v_{i} v_{j}}, c_{\beta_{i, s}}^{v_{i} v_{s}}$, and $c_{\beta j, s}^{v_{j} v_{s}}$, for each triple $\{i, j, s\}$ of $\mathscr{F}_{0}$. For a triple $e$ we sometimes write $e \in \mathscr{F}$ for $e \in E(\mathscr{F})$.

We denote by $\left(\underset{\mathscr{A}}{\mathscr{F}_{0}}\right)$ the set of copies of $\mathscr{F}_{0}$ in $\mathscr{A}$. Note that the triples in a copy of $\mathscr{F}_{0}$ in $\mathscr{A}$ may have various weights.

A fractional $\mathscr{F}_{0}$-packing of $\mathscr{A}$ is a function $\phi_{\mathscr{A}}:\left(\mathscr{\mathscr { E }}_{0}\right) \rightarrow[0,1]$, which satisfies $\Sigma_{\mathscr{G}}$ $\phi_{\mathscr{A}}(\mathscr{F}) \leq \omega(e)$ for every $e \in E(\mathscr{A})$. We let $\left|\phi_{\mathscr{A}}\right|=\sum_{\mathscr{F}} \in\left(\begin{array}{l}\mathscr{A} \\ \mathscr{F}_{0}\end{array}\right) \phi_{\mathscr{A}}(\mathscr{F})$, and we say that $\phi_{\mathscr{A}}$ is a maximum fractional $\mathscr{\Phi}_{0}$-packing of $\mathscr{A}$ if $\left|\phi_{\mathscr{A}}\right|$ is as large as possible. We denote by $\nu_{\mathscr{F}_{0}}^{*}(\mathscr{A})$ the value $\left|\phi_{\mathscr{A}}\right|$ of a maximum fractional $\mathscr{F}_{0}$-packing $\phi_{\mathscr{A}}$ of $\mathscr{A}$. 
With these definitions established, we are now able to give an informal sketch of the proof of Theorem 1.1. All details will be made precise in the rest of this section and in Section 4 . All constants referred to in this sketch depend only on the given hypergraph $\mathscr{F}_{0}$ and the error $\eta$.

\section{Sketch of the proof}

1. Given the fixed hypergraph $\mathscr{F}_{0}$ and the hypergraph $\mathscr{H}$, let $\psi$ be a maximum fractional $\mathscr{F}_{0}$-packing of $\mathscr{H}$. Find a partition 2 of the vertex set of $\mathscr{H}$ into a constant number of classes, such that most of the value $|\psi|$ comes from copies of $\mathscr{F}_{0}$ that have at most one vertex in each class (cf. Lemma 3.3).

2. Apply the hypergraph regularity lemma (Theorem 2.11) to $\mathscr{H}$, to find a $(\delta, r)$ regular $(\ell, t, \gamma, \boldsymbol{\epsilon})$-partition $\mathscr{P}$ of $\mathscr{H}$ that respects 2 . Let $m$ denote the size of the vertex classes of $\mathscr{P}$.

3. Construct an $\ell$-augmented weighted 3 -graph $\mathscr{A}$ that has a vertex for each vertex class of $\mathscr{P}$, and such that each pair of vertices $v$ and $w$ is joined by $\ell_{v w} \leq \ell$ pairs $c_{i}^{v w}$, one for each $\epsilon$-regular bipartite graph $P_{i}^{v w}$ that joins the vertex classes corresponding to $v$ and $w$. We then let $e=\left\{c_{i}^{v w}, c_{j}^{v x}, c_{k}^{w x}\right\}$ form a triple of $\mathscr{A}$ if and only if the corresponding triad $P_{e}=\left(P_{i}^{v w}, P_{j}^{v x}, P_{k}^{w x}\right)$ is such that $\mathcal{H}\left(P_{e}\right)$ is $(\delta$, $r)$-regular with respect to $P_{e}$, and we weight the triple $e$ with the density of $\mathscr{H}\left(P_{e}\right)$. (See Definition 3.9.) It can then be shown that Step 1 implies $\nu_{\mathscr{F}_{0}}^{*}(\mathscr{A})(m / \ell)^{3}$ is nearly as large as $|\psi|=\nu_{\mathscr{F}_{0}}^{*}(\mathscr{H})$ (cf. Lemma 3.10).

4. Find a fractional $\mathscr{F}_{0}$-packing $\phi_{\mathscr{A}}$ of $\mathscr{A}$ whose value is close to $\nu_{\mathscr{F}_{0}}^{*}(\mathscr{A})$, with the additional property that there exists some constant $\tau$ which is not too small, such that $\phi_{\mathscr{A}}(\mathscr{F})>\tau$ whenever $\phi_{\mathscr{A}}(\mathscr{F})>0$ (cf. Lemma 3.6).

Our aim is to "lift" the fractional $\mathscr{F}_{0}$-packing $\phi_{\mathscr{A}}$ of $\mathscr{A}$ to an integer $\mathscr{F}_{0}$-packing of $\mathscr{H}$, of value approximately $\left|\phi_{\mathscr{A}}\right|(m / \ell)^{3}$. To do this, we want to find for each $\mathscr{E} \in$ $\left(\begin{array}{c}\mathscr{A} \\ \mathscr{F}_{0}\end{array}\right)$ a blown-up copy of $\mathscr{F}_{0}$ in $\mathscr{H}$ of density $\phi_{\mathscr{A}}(\mathscr{F})$, such that all these blown-up copies are triple-disjoint.

5. For each triple $e$ of $\mathscr{A}$, let $\mathscr{D}(e)=\left\{\mathscr{F} \in\left(\mathscr{\mathscr { A }}_{0}\right): e \in \mathscr{F}, \phi_{\mathscr{A}}(\mathscr{F})>0\right\}$. We can think of $\phi_{\mathscr{A}}$ as placing a set of "demands" $\left\{\phi_{\mathscr{A}}(\mathscr{F}): \mathscr{\mathscr { F }} \in \mathscr{D}(e)\right\}$ on $e$, where the sum of these demands is at most $\omega(e)$. The triple $e$ corresponds to a $(\delta, r)$-regular 3-partite 3 -cylinder $\mathscr{H}\left(P_{e}\right)$ of density $\omega(e)$. We "slice" $\mathscr{H}\left(P_{e}\right)$ into $|\mathscr{D}(e)|$ triple-disjoint $(2 \delta$, $r$ )-regular 3-partite 3-cylinders on $P_{e}$, one for each $\mathscr{F} \in \mathscr{D}(e)$, of densities $\phi_{\mathscr{A}}(\mathscr{F})$ (cf. Lemma 3.5). The idea here is that each $\mathscr{E} \in \mathscr{D}(e)$ then receives a "share" from $\mathscr{H}\left(P_{e}\right)$ that satisfies its demand, where a share is a $(2 \delta, r)$-regular 3-partite 3 -cylinder of density $\phi_{\mathscr{A}}(\mathscr{F})$. [Step 4 guarantees that the densities $\phi_{\mathscr{A}}(\mathscr{F})$ are larger than $\tau>2 \delta$, and hence the regularity of these 3-partite 3-cylinders is nontrivial.] After this is repeated for each triple $e$ in $\mathscr{A}$, each $\mathscr{E} \in\left(\begin{array}{c}\mathscr{A} \\ \mathscr{F}_{0}\end{array}\right)$ for which $\phi_{\mathscr{A}}(\mathscr{F})>0$ has received its share of $\mathscr{H}\left(P_{e}\right)$ for every $e \in \mathscr{F}$. In other words, corresponding to each such $\mathscr{F}$, we have a $(2 \delta, r)$-regular 3 -partite 3 -cylinder of density $\phi_{\mathscr{A}}(\mathscr{F})$ in $\mathscr{H}$ for every triple $e$ of $\mathscr{g}$. Then the union of these 3-partite 3-cylinders over all triples $e$ of $\mathscr{F}$ forms a blown-up copy of $\mathscr{F}_{0}$ in $\mathcal{H}$ of density $\phi_{\mathscr{A}}(\mathscr{F})$. Moreover, these blown-up copies are all triple-disjoint for different $\mathscr{F}$. See Stage A of the proof of Theorem 3.11 (Section 4) for the precise description of this step.

6. Now by the fact mentioned at the beginning of this section, in each blown-up copy of $\mathscr{F}_{0}$ we can find a nearly-perfect $\mathscr{F}_{0}$-packing (cf. Lemma 3.4). This is done in Stage $\mathrm{B}$ of the proof. 
7. The union of all these packings over all $\mathscr{F} \in\left(\underset{\mathscr{F}_{0}}{\mathscr{A}}\right)$ gives a $\mathscr{F}_{0}$-packing of size approximately $\left|\phi_{\mathscr{A}}\right|(m / \ell)^{3}$ in $\mathscr{H}$. This shows that $\nu_{\mathscr{F}_{0}}(\mathscr{H})$ is nearly as large as $\nu_{\mathscr{F}_{0}}^{*}(\mathscr{A})(m / \ell)^{3}$. See Stage $\mathrm{C}$ of the proof.

To describe the construction of $\mathscr{A}$ we first need to establish four lemmas.

For a 3-uniform hypergraph $\mathscr{H}$ and a partition $\mathscr{2}=\left(V_{i}\right)_{i=1}^{k}$ of the vertex set $V(\mathscr{H})$ of $\mathscr{H}$, we say that a subhypergraph $\mathscr{F}$ of $\mathscr{H}$ is crossing in $\mathscr{2}$ if no two vertices of $\mathscr{F}$ are in the same class $V_{i}$ of 2 . We write $\mathscr{C}\left(\mathscr{H}, 2, \mathscr{F}_{0}\right)$ for the set of copies of $\mathscr{F}_{0}$ in $\mathscr{H}$ which are crossing in 2. Corresponding to Step 1 in the Sketch of Proof, we have the following lemma. Its proof can be found in Section 5.

Lemma 3.3. Let $\lambda>0$ and a hypergraph $\mathscr{g}_{0}$ be given. Then there exists $K=K\left(\lambda, \mathscr{F}_{0}\right)$ such that the following holds.

Let $\mathcal{H}$ be a 3-uniform hypergraph with $n$ vertices, and let $\psi^{*}$ be a fractional $\mathscr{F}_{0}$-packing of $\mathcal{H}$. Then there exists a partition $\mathscr{2}=\left(V_{i}\right)_{i=1}^{q}$ of $V(\mathcal{H})$, where $q \leq K$, such that

(i) $\lfloor n / q\rfloor \leq\left|V_{i}\right| \leq\lceil n / q\rceil$ for $1 \leq i \leq q$,

(ii) $\sum_{\mathscr{F} \in \mathscr{C}\left(\mathscr{H}, \mathscr{2}, \mathscr{F}_{0}\right)} \psi^{*}(\mathscr{F}) \geq(1-\lambda) \sum_{\mathscr{F} \in(}\left(\begin{array}{l}\mathscr{H} \\ \mathscr{F}_{0}\end{array}\right) \psi^{*}(\mathscr{\mathscr { F }})$.

Let $\mathscr{F}_{0}$ be a 3 -uniform hypergraph with vertices $1, \ldots, k$ that are ordered by 1 $<\cdots<k$. Let $\mathscr{H}$ be a $k$-partite 3 -cylinder with vertex classes $V_{1}, \ldots, V_{k}$. Then we say that a sub-hypergraph $\mathscr{\mathscr { F }}$ of $\mathscr{H}$ with ordered vertex set $v_{1}<\cdots<v_{k}$ is partite-isomorphic to $\mathscr{F}_{0}$ in $\mathscr{H}$ if $v_{i} \in V_{i}$ for each $i$ and the map $v_{i} \rightarrow i, 1 \leq i \leq k$, is an isomorphism from $\mathscr{F}$ to $\mathscr{F}_{0}$. The following lemma is needed for Step 6, and is proved in Section 8 .

Lemma 3.4. Let $\mathscr{F}_{0}$ be a 3-uniform hypergraph with vertices $1, \ldots, k$. Let real numbers $\lambda>0$ and $\alpha>0$ be given. Then there exists $\theta=\theta\left(\mathscr{\Phi}_{0}, \lambda, \alpha\right)>0$ such that for all integers $\ell>1 / \theta$, there exist $r_{0}\left(\mathscr{F}_{0}, \lambda, \alpha, \ell\right), \epsilon_{0}\left(\mathscr{F}_{0}, \lambda, \alpha, \ell\right)$, and $m_{0}=m_{0}\left(\mathscr{F}_{0}, \lambda, \alpha, \ell\right)$ such that the following holds. Let $\mathscr{H}$ and $G$ be such that

(i) $\mathscr{H}$ is a k-partite 3-cylinder with $k$-partition $V_{1}, \ldots, V_{k},\left|V_{1}\right|=\cdots=\left|V_{k}\right| \geq m_{0}$,

(ii) $G=\bigcup_{1 \leq i<j \leq k} G^{i j}$ is an underlying $\left(\ell, \epsilon_{0}, k\right)$-cylinder,

(iii) for all triples $\{i, j, s\}$ of $\mathscr{F}_{0}, \mathscr{H}\left(G^{i j}, G^{i s}, G^{j s}\right)$ is $\left(\alpha, \theta, r_{0}\right)$-regular with respect to $\left(G^{i j}, G^{i s}, G^{j s}\right)$.

Then $\mathcal{H}$ contains a family of triple-disjoint sub-hypergraphs of $\mathscr{H}$, each of which is partite-isomorphic to $\mathscr{F}_{0}$, which covers all but at most $\lambda|\mathscr{H}|$ triples of $\mathscr{H}$.

The next lemma corresponds to Step 5 in the Sketch. Its proof appears in Section 6.

Lemma 3.5. Let real numbers $\alpha>0,0<\delta<\alpha / 4, \epsilon>0$ and positive integers $j \leq 1 / \alpha$, $\ell$ and $r$ be given, where $\epsilon<\epsilon_{1}(\ell, 1 / 2)$ (see Fact 2.3). Then there exists $m_{1}=m_{1}(\alpha, \delta, \ell$, $r)$ such that the following holds. Let $\mathscr{H}$ be a $(\beta, \delta, r)$-regular 3-partite 3-cylinder with respect to an underlying $(\ell, \epsilon, 3)$-cylinder $G$, where $j \alpha \leq \beta<(j+1) \alpha$. Let the vertex classes of $\mathcal{H}$ be $V_{1}, V_{2}$ and $V_{3}$, where $\left|V_{1}\right|=\left|V_{2}\right|=\left|V_{3}\right| \geq m_{1}$. Then there exist $j$ triple-disjoint sub-hypergraphs $\mathscr{H}_{1}, \ldots, \mathscr{H}_{j}$ of $\mathscr{H}$, such that each $\mathscr{H}_{i}$ is $(\alpha, 2 \delta, r)$-regular with respect to $G$. 
For an $\ell$-augmented weighted 3-graph $\mathscr{A}$ and a real number $\tau>0$, we say a fractional $\mathscr{F}_{0}$-packing $\phi_{\mathscr{A}}$ of $\mathscr{A}$ is $\tau$-bounded if for each $\mathscr{F} \in\left(\begin{array}{l}\mathscr{A} \\ \mathscr{F}_{0}\end{array}\right)$, either $\phi_{\mathscr{A}}(\mathscr{F})=0$ or $\phi_{\mathscr{A}}(\mathscr{F}) \geq \tau$. The following lemma proves the existence of the fractional $\mathscr{F}_{0}$-packing used in Step 4, and its proof is in Section 7.

Lemma 3.6. Let a 3-uniform hypergraph $\mathscr{F}_{0}$ and a real number $\eta>0$ be given. Then there exists $\tau=\tau\left(\mathscr{F}_{0}, \eta\right)$ such that the following holds. For every $\ell$-augmented weighted 3-graph $\mathscr{A}$ with $t$ vertices, there exists a $\tau$-bounded fractional $\mathscr{F}_{0}$-packing $\phi_{\mathscr{A}}$ of $\mathscr{A}$ such that $\left|\phi_{\mathscr{A}}\right| \geq \nu_{\mathscr{F}_{0}}^{*}(\mathscr{A})-\eta \ell^{3} t^{3}$.

We now define the constants we shall require for our application of the Hypergraph Regularity Lemma. Recall that we are given an error term $\eta>0$ and a fixed 3-uniform hypergraph $\mathscr{F}_{0}$.

\section{Definition 3.7.}

(i) Let $\tau=\tau\left(\eta / 100, \mathscr{E}_{0}\right)($ see Lemma 3.6).

(ii) Let $\alpha=\tau \eta / 100$.

(iii) Let $\delta=\min \left\{\theta\left(\mathscr{\Phi}_{0}, \eta / 100, \alpha\right) / 4, \alpha \eta / 100\right\}$ (see Lemma 3.4).

(iv) Let $\gamma=\min \left\{\eta / 100,2 \delta^{4}\right\}$.

(v) Let $q=K\left(\eta / 100, \mathscr{F}_{0}\right)($ see Lemma 3.3).

(vi) Let $t_{0}=100 / \eta$.

We also define two functions:

(vii) Let $r(\ell)=r_{0}\left(\Phi_{0}, \eta / 100, \alpha, \ell\right)$ (see Lemma 3.4).

(viii) Let $\epsilon(\ell)=\min \left\{\epsilon_{0}\left(\Phi_{0}, \eta / 100, \alpha, \ell\right), \epsilon_{1}(\ell, \eta / 100)\right\}$ (see Lemma 3.4 and Fact 2.3).

Let $T_{0}, L_{0}$ and $N_{0}$ be the constants guaranteed by Lemma 2.11 with input constants $\delta$, $\gamma, q, t_{0}$, and $\ell_{0}=1$, and functions $r(\ell)$ and $\epsilon(\ell)$ as in Definition 3.7. Finally we let $N_{1}=$ $\max \left\{N_{0}, 100 T_{0} / \eta, T_{0} m_{0}\left(\Phi_{0}, \eta / 100, \alpha, L_{0}\right), T_{0} m_{1}\left(\alpha, \delta, L_{0}, r\left(L_{0}\right)\right)\right\}$ (see Theorem 2.11 and Lemmas 3.4 and 3.5).

Then these constants satisfy the following hierarchy.

$$
\frac{1}{N_{1}} \ll \epsilon \ll \frac{1}{r}, \frac{1}{T_{0}}, \frac{1}{L_{0}} \ll \delta \ll \alpha<\tau, \gamma, \frac{1}{q}, \frac{1}{t_{0}}<\eta .
$$

We are now ready to describe how to construct the finite $\ell$-augmented weighted 3 -graph $\mathscr{A}$ from a given 3 -uniform hypergraph $\mathscr{H}$. Here $\ell$ will be at most $L_{0}$. Recall that $\mathscr{A}$ will be an analogue of the "cluster graph" that results from the application of Szemerédi's Regularity Lemma for graphs. We will obtain $\mathscr{A}$ by applying the hypergraph regularity lemma (Theorem 2.11) to $\mathscr{H}$ together with a vertex partition 2 defined as follows.

Definition 3.8. Let a 3-uniform hypergraph He given, where the number of vertices of $\mathcal{H}$ is at least $N_{1}$. Let $\psi$ be a maximum fractional $\mathscr{F}_{0}$-packing of $\mathcal{H}$. Then by Lemma 3.3 
applied to $\mathscr{H}$ with $\lambda=\eta / 100$, there exists a vertex partition 2 of $\mathscr{H}$ with $q \leq K(\eta / 100$, $\mathscr{g}_{0}$ ) parts such that

$$
\sum_{\mathscr{F} \in \mathscr{C}\left(\mathscr{H}, \mathscr{2}, \mathscr{F}_{0}\right)} \psi(\mathscr{F}) \geq(1-\eta / 100)|\psi|=(1-\eta / 100) \nu_{\mathscr{F}_{0}}^{*}(\mathscr{H}) .
$$

Now we apply Theorem 2.11 to our given hypergraph $\mathscr{H}$ with the partition 2 and with constants and functions as above, to obtain an $(\ell, t, \gamma, \epsilon)$-partition $\mathscr{P}$ as in Definition 2.4 that respects 2 . We will concentrate on a subhypergraph $\mathscr{H}^{\prime}$ of $\mathscr{H}$, consisting of those triples of $\mathscr{H}$ which "conform" to the regularity properties. The vertex set of $\mathscr{H}^{\prime}$ will be $V_{1}$ $\cup \cdots \cup V_{t}$. Let $\mathscr{B}^{\prime} \subset \mathscr{B}$ be the subset consisting of those bipartite graphs $P_{\beta}^{i j}, \beta \geq 1$, that are $\epsilon$-regular, and where the pair $i, j$ satisfies Part (iii) of Definition 2.4. We then let $\mathscr{H}^{\prime}$ be the subhypergraph of $\mathscr{H}$ consisting of those triples which lie in $(\delta, r)$-regular 3 -partite 3-cylinders of $\mathscr{H}$, and whose underlying bipartite graphs in $\mathscr{P}$ are all elements of $\mathscr{B}^{\prime}$.

We may now define the $\ell$-augmented weighted 3-graph $\mathscr{A}=\mathscr{A}(\mathscr{H})$.

Definition 3.9. With the above definitions, we define $\mathscr{A}$ as follows:

(i) Let the vertex set $V(\mathscr{A})=\left\{v_{1}, \ldots, v_{t}\right\}$.

(ii) Let the pair multiset $C(\mathscr{A})=\left\{c_{\beta}^{v_{i} v_{s}}: P_{\beta}^{v_{i} v_{s}} \in \mathscr{B}^{\prime}\right\}$, where $c_{\beta}^{v_{i} v_{s}}$ joins vertices $v_{i}$ and $v_{s}$.

(iii) Let the triple set $E(\mathscr{A})$ contain one triple $\left\{c_{\beta_{1}}^{v_{i} v_{s}}, c_{\beta_{2}}^{v_{i} v_{h}}, c_{\beta_{3}}^{v_{h} v_{s}}\right\}$ for each triad $P=\left(P_{\beta_{1}}^{v_{i} v_{s}}, P_{\beta_{2}}^{v_{i} v_{h}}\right.$, $\left.P_{\beta_{3}}^{v_{h} v_{s}}\right)$ with the property that $\mathcal{H}^{\prime}(P)$ is $(\delta, r)$-regular with respect to $P$. We assign weight $\omega\left(\left\{c_{\beta_{1}}^{v_{i} v_{s}}, c_{\beta_{2}}^{v_{i} v_{h}}, c_{\beta_{3}}^{v_{h} v_{s}}\right\}\right)=\mu$, where $\mu$ is the density of $\mathcal{H}^{\prime}(P)$.

Then we have the following (see Step 3 of the Sketch of Proof).

Lemma 3.10. With the above definitions we have

(i) $|\mathscr{H}|-\left|\mathscr{H}^{\prime}\right|<\eta n^{3} / 25$,

(ii) $\nu_{\mathscr{F}_{0}}^{*}(\mathscr{H}) \leq(m / \ell)^{3} \nu_{\mathscr{F}_{0}}^{*}(\mathscr{A})+3 \eta n^{3} / 50$.

The proof of this lemma appears in Section 9.

To prove Theorem 1.1, we shall instead prove the following result, which immediately implies Theorem 1.1.

Theorem 3.11. With the above definitions we have

(i) $\nu_{\mathscr{F}_{0}}^{*}(\mathscr{H})<(m / \ell)^{3} \nu_{\mathscr{F}_{0}}^{*}(\mathscr{A})+\eta n^{3} / 2$,

(ii) $\nu_{\mathscr{F}_{0}}(\mathscr{H}) \geq \nu_{\mathscr{F}_{0}}\left(\mathscr{H}^{\prime}\right)>(m / \ell)^{3} \nu_{\mathscr{F}_{0}}^{*}(\mathscr{A})-\eta n^{3} / 2$.

The proof of Theorem 1.1 is immediate from Theorem 3.11, since $\nu_{\mathscr{F}_{0}}(\mathscr{H})>$ $(m / \ell)^{3} \nu_{\mathscr{F}_{0}}^{*}(\mathscr{A})-\eta n^{3} / 2>\nu_{\mathscr{F}_{0}}^{*}(\mathscr{H})-\eta n^{3}$. 


\section{PROOF OF THEOREM 3.11}

Part (i) of Theorem 3.11 is immediate from (ii) of Lemma 3.10. Therefore, we consider the proof of Part (ii). Recall that $\mathscr{A}$ is the $\ell$-augmented weighted 3 -graph defined in Definition 3.9, and the constants and integer-valued functions we refer to here are as defined in Definition 3.7. Our aim is to find an $\mathscr{F}_{0}$-packing in $\mathscr{H}^{\prime}$ of size approximately $\nu_{\mathscr{F}_{0}}^{*}(\mathscr{A})(m / \ell)^{3}$. (See the paragraph before Definition 3.9 for the definition of $\mathscr{H}^{\prime}$.)

First we establish that since $\tau=\tau\left(\mathscr{E}_{0}, \eta / 100\right)$ [see Definition 3.7 (i)], by Lemma 3.6 there exists a $\tau$-bounded fractional $\mathscr{F}_{0}$-packing $\phi_{\mathscr{A}}$ of $\mathscr{A}$ satisfying

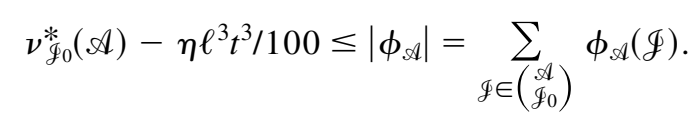

Then for every $e \in E(\mathscr{A})$ we have

$$
\sum_{\mathscr{g} \ni e} \phi_{\Re}(\mathscr{F}) \leq \omega(e)
$$

and for each $\mathscr{E} \in\left(\underset{\mathscr{F}_{0}}{\mathscr{A}}\right)$ we have

$$
\phi_{\mathscr{A}}(\mathscr{F})=0 \quad \text { or } \quad \phi_{\mathscr{A}}(\mathscr{F}) \geq \tau
$$

The rest of the proof will consist of three stages, corresponding to Steps 5, 6, and 7 of the Sketch of Proof.

Stage A. The aim of Stage A is to execute Step 5 of the Sketch, that is, for each triple $e$ of $\mathscr{A}$ we "slice" $\mathscr{H}^{\prime}\left(P_{e}\right)$ into 3-partite 3-cylinders of densities the various demands on $e$. To do this, it will be convenient for us to first slice all $\mathscr{H}^{\prime}\left(P_{e}\right)$ into 3-partite 3-cylinders of the same small density $\alpha$, which we call elementary. Then for each $\mathscr{E} \in\left(\begin{array}{l}\mathscr{A} \\ \mathscr{F}_{0}\end{array}\right)$ that contains $e$, we assign to $\mathscr{F}$ its share $\left\lfloor\frac{\phi_{\mathcal{A}}(\mathcal{F})}{\alpha}\right\rfloor$ of the elementary 3-partite 3-cylinders of $\mathcal{H}^{\prime}\left(P_{e}\right)$.

Let $e=\left\{c_{\beta_{1}}^{v_{i} v_{s}}, c_{\beta_{2}}^{v_{i} v_{h}}, c_{\beta_{3}}^{v_{s} v_{h}}\right\} \in E(\mathscr{A})$ be a triple of $\mathscr{A}$. Then $P_{e}=\left(P_{\beta_{1}}^{v_{i} v_{s}}, P_{\beta_{2}}^{v_{i} v_{h}}, P_{\beta_{3}}^{v_{s} v_{h}}\right)$

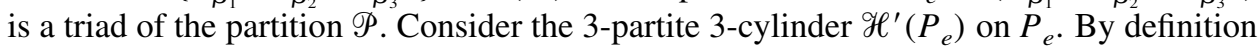
of $\mathscr{A}$, we know that $\mathscr{H}^{\prime}\left(P_{e}\right)$ is $(\omega(e), \delta, r)$-regular, and has vertex classes $V_{i} \cup V_{h} \cup V_{s}$. We wish to apply Lemma 3.5 to $\mathcal{H}^{\prime}\left(P_{e}\right)$ with parameters $\alpha, \delta, \epsilon, \ell$ and $r$ as defined in Definition 3.7, and with $j=j_{e}=\lfloor\omega(e) / \alpha\rfloor$. Note that by definition of $N_{1}$ (Definition 3.7) we have $\left|V_{i}\right|=\left|V_{h}\right|=\left|V_{s}\right|=\lfloor n / t\rfloor \geq N_{1} / T_{0} \geq m_{1}(\alpha, \delta, \epsilon, \ell)$. Therefore, we may apply Lemma 3.5 to $\mathscr{H}^{\prime}\left(P_{e}\right)$ to find $j_{e}$ triple-disjoint 3-partite 3-cylinders $\mathscr{H}^{\prime}\left(P_{e}\right)_{1}, \ldots$, $\mathcal{H}^{\prime}\left(P_{e}\right)_{j_{e}}$ on the triad $P_{e}$ such that each is $(\alpha, 2 \delta, r)$-regular. These subcylinders of $\mathscr{H}^{\prime}\left(P_{e}\right)$ are what we call elementary 3-partite 3-cylinders of $\mathcal{H}^{\prime}\left(P_{e}\right)$.

Now we assign to each $\mathscr{F} \in\left(\mathscr{\mathscr { F }}_{0}\right)$ containing $e$ the appropriate number $\left\lfloor\frac{\phi_{\mathscr{s}}(\mathcal{F})}{\alpha}\right\rfloor$ of elementary 3-partite 3-cylinders of $\mathscr{H}^{\prime}\left(P_{e}\right)$. Let $\mathscr{F}$ be fixed. Then we assign to $\mathscr{F}$ a set $S_{e}(\mathscr{F})$ of $\left\lfloor\frac{\phi_{\mathcal{A}}(\mathscr{\mathscr { F }})}{\alpha}\right\rfloor$ elementary 3-partite 3-cylinders of $\mathscr{H}^{\prime}\left(P_{e}\right)$, such that the $\left\{S_{e}(\mathscr{F})\right\}_{\mathscr{F} \ni e}$ are all triple-disjoint. This is possible since by (5), $\sum_{\mathscr{F} \ni e}\left\lfloor\phi_{\mathscr{A}}(\mathscr{F}) / \alpha\right\rfloor \leq\lfloor\omega(e) / \alpha\rfloor \leq j_{e}$. We repeat this for every triple $e$ of $\mathscr{A}$. This completes Stage A. 
Stage B. In this stage, we execute Step 6 of the Sketch by finding a nearly-perfect packing inside the blown-up copy of $\mathscr{F}_{0}$ corresponding to each $\mathscr{F} \in\left(\underset{\mathscr{F}}{\mathscr{F}_{0}}\right)$. In fact, we will treat

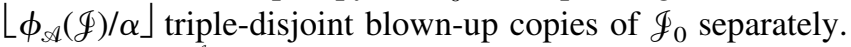

Let $\mathscr{F} \in\left(\underset{\mathscr{F}_{0}}{\mathscr{A}}\right)$ such that $\phi_{\mathscr{A}}(\mathscr{F})>0$ be fixed. In Stage A, $\mathscr{F}$ was assigned the set $S_{e}(\mathscr{F})$ of $\left\lfloor\frac{\phi_{s t}(\mathscr{F})}{\alpha}\right\rfloor$ elementary 3-partite 3-cylinders of $\mathscr{H}^{\prime}\left(P_{e}\right)$ with underlying cylinder $P_{e}$ for each triple $e$ of $\mathscr{F}$. We order each $S_{e}(\mathscr{F})$ arbitrarily, say $S_{e}(\mathscr{F})=\left(\mathscr{H}^{\prime}\left(P_{e}\right)_{i_{\mathscr{F} 1}}, \ldots\right.$, $\left.\mathscr{H}^{\prime}\left(P_{e}\right)_{\left.i_{\mathscr{q}\left\lfloor\phi_{\mathcal{S}}(\mathscr{q}) / \alpha\right.}\right)}\right)$.

Fix $b$ with $1 \leq b \leq\left\lfloor\frac{\phi_{s \mathcal{L}}(\mathscr{f})}{\alpha}\right\rfloor$. Let $\mathscr{L}=\mathscr{L}(\mathscr{F}, b)=\bigcup_{e \in \mathscr{F}} \mathscr{H}^{\prime}\left(P_{e}\right)_{i \mathscr{}}$ (so $\mathscr{L}$ is a $k$-partite 3 -cylinder in $\mathscr{H}^{\prime}$ formed by placing the $b$ th elementary 3-partite 3 -cylinder assigned to $\mathscr{F}$ on each triad $P_{e}$ for which $e$ is a triple of $\mathscr{F}$ in $\mathscr{A}$ ). Then $\mathscr{L}$ is a blown-up copy of $\mathscr{F}_{0}$ in $\mathscr{H}^{\prime}$, of density $\alpha$. Since each vertex class $V_{i}$ of $\mathscr{L}$ has size $\lfloor n / t\rfloor \geq N_{1} / T_{0} \geq m_{0}\left(\mathscr{E}_{0}, \eta / 100, \alpha\right.$, $\ell$ ) (see the definition of $N_{1}$ in Definition 3.7) and $2 \delta<\theta\left(\mathscr{F}_{0}, \eta / 100, \alpha\right)$, and by definition of $r$ and $\epsilon$ [see Definition 3.7 (iii), (vii), (viii)], we may apply Lemma 3.4 to $\mathscr{L}$ with parameters $\lambda=\eta / 100$ and $\alpha$ as in Definition 3.7 to find a family $\mathscr{T}(\mathscr{F}, b)$ of triple-disjoint copies of $\mathscr{F}_{0}$ in $\mathscr{L}$ that covers at least $(1-\eta / 100)|\mathscr{L}|$ triples of $\mathscr{L}$. By definition of $\mathscr{L}$ and of $\epsilon$ (see Fact 2.3), we find that $|\mathscr{L}| \geq(1-\eta / 100)\left|\mathscr{I}_{0}\right|(\alpha-2 \delta)(m / \ell)^{3}$ and hence $|\mathscr{T}(\mathscr{F}, b)| \geq(1-\eta / 100)^{2}(\alpha-$ $2 \delta)(m / \ell)^{3}>(1-\eta / 50)(\alpha-2 \delta)(m / \ell)^{3}$.

Since all the elementary 3-partite 3-cylinders were triple-disjoint, we can construct a set of $\left\lfloor\frac{\phi_{\mathscr{A}}(\mathscr{F})}{\alpha}\right\rfloor$ triple-disjoint 3-cylinders $\left\{\mathscr{L}(\mathscr{F}, b), 1 \leq b \leq\left\lfloor\frac{\phi_{\mathscr{A}}(\mathscr{\mathscr { F }})}{\alpha}\right\rfloor\right\}$ of this type associated with $\mathscr{F}$. Therefore, we find altogether a $\mathscr{F}_{0}$-packing $\mathscr{T}(\mathscr{F})=\bigcup_{1 \leq b \leq\left\lfloor\phi_{\mathscr{A}}(\mathscr{F}) / \alpha\right\rfloor} \mathscr{T}(\mathscr{F}, b)$ in $\mathscr{H}^{\prime}$ corresponding to $\mathscr{E}$, where

$$
|\mathscr{T}(\mathscr{F})| \geq\left(1-\frac{\eta}{50}\right)\left\lfloor\frac{\phi_{\mathscr{A}}(\mathscr{\mathscr { S }})}{\alpha}\right\rfloor(\alpha-2 \delta)\left(\frac{m}{\ell}\right)^{3} .
$$

This completes Stage B.

Stage C. In this final stage we complete Step 7 of the Sketch by taking the union of all $\mathscr{F}_{0}$-packings $\mathscr{T}(\mathscr{F})$ found in Stage B, over all $\mathscr{F} \in\left(\begin{array}{l}\mathscr{A} \\ \mathscr{F}_{0}\end{array}\right)$.

For $\mathscr{\mathscr { S }} \in\left(\begin{array}{l}\mathcal{A} \\ \mathscr{F}_{0}\end{array}\right)$, by (6) we have $\left\lfloor\frac{\phi_{\mathscr{S A}}(\mathscr{F})}{\alpha}\right\rfloor \geq \frac{\phi_{\mathscr{S A}}(\mathscr{F})}{\alpha}(1-\alpha / \tau) \geq \frac{\phi_{\mathscr{S A}}(\mathscr{f})}{\alpha}(1-\eta / 100)$, where we use the definition of $\alpha$. Therefore, summing over all $\mathscr{E} \in\left(\begin{array}{c}\mathscr{A} \\ \mathscr{F}_{0}\end{array}\right)$ and using (7) we find a $\mathscr{F}_{0}$-packing $\bigcup_{\mathscr{I} \in\left(\begin{array}{l}\mathscr{A} \\ \mathscr{F}_{0}\end{array}\right)} \mathscr{T}(\mathscr{F})$ in $\mathscr{H}^{\prime}$ where

$$
\begin{aligned}
& \left|\bigcup_{\mathscr{F} \in\left(\begin{array}{c}
\mathscr{A} \\
\mathscr{F}_{0}
\end{array}\right)} \mathscr{T}(\mathscr{F})\right| \geq\left(1-\frac{\eta}{50}\right) \sum_{\mathscr{F} \in\left(\begin{array}{c}
\mathscr{A} \\
\mathscr{F}_{0}
\end{array}\right)}\left\lfloor\frac{\phi_{\mathscr{A}}(\mathscr{F})}{\alpha}\right\rfloor(\alpha-2 \delta)\left(\frac{m}{\ell}\right)^{3} \\
& \geq\left(1-\frac{\eta}{100}\right)\left(1-\frac{\eta}{50}\right)\left(1-\frac{2 \delta}{\alpha}\right) \sum_{\mathscr{g} \in\left(\begin{array}{l}
\mathscr{A} \\
\mathscr{F}_{0}
\end{array}\right)} \phi_{\mathscr{A}}(\mathscr{F})\left(\frac{m}{\ell}\right)^{3} \\
& >\left(1-\frac{3 \eta}{100}-\frac{2 \delta}{\alpha}\right)\left(\frac{m}{\ell}\right)^{3}\left|\phi_{\mathscr{A}}\right| \\
& \geq\left(1-\frac{3 \eta}{1000}-\frac{2 \delta}{\alpha}\right)\left(\frac{m}{\ell}\right)^{3}\left(\nu_{g_{0}}^{*}(\mathscr{A})-\frac{\eta \ell^{3} t^{3}}{100}\right),
\end{aligned}
$$


where the last line follows from (4). Then using the definition of $\delta$ (Definition 3.7 (iii)) and the fact that $m^{3} t^{3} \leq n^{3}$ and (trivially) $\nu_{\mathscr{F}_{0}}^{*}(\mathscr{A})<\ell^{3} t^{3}$, we conclude that $\nu_{\mathscr{F}_{0}}(\mathscr{H}) \geq$ $(m / \ell)^{3} \nu_{\mathscr{F}_{0}}^{*}(\mathscr{A})-\eta(m \ell t)^{3} / 20 \ell^{3}-\eta m^{3} t^{3} / 100>(m / \ell)^{3} \nu_{\mathscr{F}_{0}}^{*}(\mathscr{A})-\eta n^{3} / 2$ as required. This completes Stage $\mathrm{C}$ and hence the proof.

\section{PROOF OF LEMMA 3.3}

The proof of Lemma 3.3 will follow immediately from the following more general result, proved in [9]. Here for a partition $2=\left(V_{i}\right)_{i=1}^{q}$ of a set $V$, we say that a subset $S$ of $V$ is crossing in 2 if $\left|S \cap V_{i}\right| \leq 1$ for each $i$, and we write $\mathscr{C}(t, V, 2)$ for the set of subsets of $V$ of size $t$ that are crossing in 2 .

Lemma 5.1. Let a positive integer $t$ and a real number $\lambda>0$ be given. Then there exists $K=K(t, \lambda)$ such that the following holds. For any set $V$ and function $f:\left(\begin{array}{l}V \\ t\end{array}\right) \rightarrow \mathbb{R}^{+}$, there exists a partition $2=\left(V_{i}\right)_{i=1}^{q}$ with $q \leq K$ such that

(i) ||$V_{i}|-| V_{j} \mid \leq 1$ for each $i, j$,

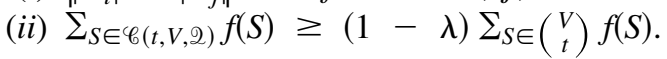

Proof of Lemma 3.3. This follows immediately from Lemma 5.1 by taking $V=V(\mathscr{H})$, $t=\left|V\left(\mathscr{F}_{0}\right)\right|$, and $f(S)=\sum_{\mathscr{F} \in(}\left(\begin{array}{l}\mathscr{H} \\ \mathscr{F}_{0}\end{array}\right): V(\mathscr{F})=S \psi^{*}(\mathscr{F})$.

\section{PROOF OF LEMMA 3.5}

We mention that this proof also appears in [12], and a similar result for graphs was given in [6].

Proof of Lemma 3.5. Let $\mathscr{H}$ be as in the statement of the lemma, and let $m=\left|V_{1}\right|=\left|V_{2}\right|$ $=\left|V_{3}\right|$. We may assume that $j \geq 2$. We set $m_{1}=m_{1}(\sigma, \delta, \ell, r)$ to be large enough such that for all $m>m_{1}$ we have

$$
2 \sigma^{-1} 2^{3 r m^{2}} \exp \left(\left[-\delta^{3} / 4 \sigma \ell^{3}\right] m^{3}\right)<1 .
$$

For each $\{x, y, z\} \in \mathscr{H}$, let $X_{\{x, y, z\}}$ be a random variable assuming values from $\{0$, $1, \ldots, j\}$ defined by

$$
\operatorname{Pr}\left(X_{\{x, y, z\}}=i\right)= \begin{cases}1-\frac{j \sigma}{\beta} & \text { if } i=0 \\ \frac{\sigma}{\beta} & \text { if } 1 \leq i \leq j .\end{cases}
$$

For each $i \in[j]$, we define a subhypergraph $\mathscr{H}_{i}$ by $\mathscr{H}_{i}=\left\{\{x, y, z\} \in \mathscr{H}_{: X_{\{x, y, z\}}=}\right.$ $i\}$. Then $\mathscr{H}=\bigcup_{i=0}^{j} \mathscr{H}_{i}$ is a partition. We will show that, for one such partition, each $\mathscr{H}_{i}, 1 \leq i \leq j$, is $(\sigma, 2 \delta, r)$-regular with respect to $G$.

Let $i \in[j]$ be fixed, and let $\vec{Q}=(Q(s)), 1 \leq s \leq r$, be an $r$-tuple of 3-partite graphs 
satisfying that for each $s \in[r], Q(s)=Q^{12}(s) \cup Q^{13}(s) \cup Q^{23}(s)$, where for each $\{j$, $k\} \in[3]^{2}, Q^{j k}(s) \subset G^{j k}$. Suppose further that

$$
\left|\bigcup_{s=1}^{r} \mathscr{K}_{3}(Q(s))\right|>2 \delta\left|\mathscr{K}_{3}(G)\right| .
$$

By the $(\beta, \delta, r)$-regularity of $\mathscr{H}$ with respect to $G$, we have

$$
(\beta-\delta)\left|\bigcup_{s=1}^{r} \mathscr{K}_{3}(Q(s))\right|<\left|\mathscr{H} \cap \bigcup_{s=1}^{r} \mathscr{K}_{3}(Q(s))\right|<(\beta+\delta)\left|\bigcup_{s=1}^{r} \mathscr{K}_{3}(Q(s))\right| .
$$

Thus, the expected number of triples of $\mathscr{H}_{i}$ which coincide with $\bigcup_{s=1}^{r} \mathscr{K}_{3}(Q(s))$ satisfies

$$
(\beta-\delta) \frac{\sigma}{\beta}\left|\bigcup_{s=1}^{r} \mathscr{K}_{3}(Q(s))\right|<\exp \left|\mathscr{H}_{i} \cap \bigcup_{s=1}^{r} \mathscr{K}_{3}(Q(s))\right|<(\beta+\delta) \frac{\sigma}{\beta}\left|\bigcup_{s=1}^{r} \mathscr{K}_{3}(Q(s))\right| .
$$

Set

$$
M=\exp \left|\mathscr{H}_{i} \cap \bigcup_{s=1}^{r} \mathscr{K}_{3}(Q(s))\right|
$$

and $\gamma=\frac{\delta}{\sigma}$, so that $\sigma+2 \delta \geq(1+\gamma)\left(\sigma+\delta \frac{\sigma}{\beta}\right)$ and $\sigma-2 \delta \leq(1-\gamma)\left(\sigma-\delta \frac{\sigma}{\beta}\right)$. Thus,

$$
\begin{aligned}
\operatorname{Pr}\left(|| \mathscr{H}_{\mathrm{i}} \cap \bigcup_{s=1}^{r} \mathscr{K}_{3}(Q(s))|-\sigma| \bigcup_{s=1}^{r} \mathscr{K}_{3}(Q(s))||\right. & \left.\geq 2 \delta\left|\bigcup_{s=1}^{r} \mathscr{K}_{3}(Q(s))\right|\right) \\
& \leq \operatorname{Pr}\left(|| \mathscr{H}_{i} \cap \bigcup_{s=1}^{r} \mathscr{K}_{3}(Q(s))|-M| \geq \gamma M\right) .
\end{aligned}
$$

Using the Chernoff inequality (see, e.g., [11]), we may further bound the quantity above by $2 \exp \left(-\gamma^{2} M / 3\right)=2 \exp \left(-\delta^{2} M / 3 \sigma^{2}\right)$. Combining (8) with the fact that $\left|\mathscr{K}_{3}(G)\right|>$ $\left(m^{3} / 2 \ell^{3}\right)$ (see Fact 2.3), we conclude that $M>(\beta-\delta) \sigma \delta m^{3} / \beta \ell^{3}>3 \sigma \delta m^{3} / 4 \ell^{3}$. Therefore,

$$
\begin{aligned}
& \operatorname{Pr}\left(|| \mathscr{H}_{i} \cap \bigcup_{s=1}^{r} \mathscr{K}_{3}(Q(s))|-\sigma| \bigcup_{s=1}^{r} \mathscr{K}_{3}(Q(s))|| \geq 2 \delta\left|\bigcup_{s=1}^{r} \mathscr{K}_{3}(Q(s))\right|\right) \\
& \quad \leq 2 \exp \left(-\left[\delta^{3} / 4 \sigma \ell^{3}\right] m^{3}\right) .
\end{aligned}
$$

As the number of $r$-tuples $\vec{Q}=Q(s), 1 \leq s \leq r$, does not exceed $2^{3 r m^{2}}$, we infer that the probability that $\mathcal{H}_{i}$ is $(\sigma, 2 \delta, r)$-regular tends to 1 as $m \rightarrow \infty$. Moreover, since there are $j \leq \sigma^{-1}$ subhypergraphs $\mathscr{H}_{i}$, we find that the probability that all are $(\sigma, 2 \delta, r)$-regular is 
positive, since $m_{1}$ was chosen to be large enough such that $2 \sigma^{-1} 2^{3 r m^{2}} \exp \left(\left[-\delta^{3} / 4 \sigma \ell^{3}\right] m^{3}\right)$ $<1$ for $m \geq m_{1}$. Therefore, there exists a choice of subhypergraphs $\mathscr{H}_{1}, \ldots, \mathscr{H}_{j}$ as required.

\section{PROOF OF LEMMA 3.6}

We shall deduce Lemma 3.6 from a more general result about fractional packings in weighted hypergraphs, which was proved in [9]. For a vertex-weighted hypergraph $\mathscr{K}$ in which every vertex $v \in V(\mathscr{K})$ receives a weight $w(v)$, we say that a function $\phi: \mathscr{K} \rightarrow$ $[0,1]$ is a fractional packing of $\mathscr{K}$ if $\Sigma_{E \ni v} \phi(E) \leq w(v)$ for every $v \in V(\mathscr{K})$. We say that a fractional packing is $\tau$-bounded for some real number $\tau$ if, for each $E \in \mathscr{K}$, either $\phi(E)=0$ or $\phi(E) \geq \tau$.

We shall use the following theorem from [9].

Theorem 7.1. Let $\eta>0$ and $b \in \mathbb{N}$ be given. Then there exists $k_{0}=k_{0}(b, \eta)$ such that the following holds. Let $\mathscr{K}$ be any b-uniform vertex-weighted hypergraph, where the weight $w(v)$ of each vertex $v$ satisfies $0 \leq w(v) \leq 1$, and let $\phi$ be any fractional packing of $\mathscr{K}$ such that $\phi(E)<1 / k_{0}$ for every $E \in \mathscr{K}$. Then there exists a $1 / k_{0}$-bounded fractional packing $\bar{\phi}$ of $\mathscr{K}$ such that $|\bar{\phi}| \geq|\phi|-\eta n$, where $|V(\mathscr{K})|=n$.

Proof of Lemma 3.6. Given $\mathscr{F}_{0}$ and $\eta$, we let $b$ denote the number of triples in $\mathscr{F}_{0}$, and we let $\tau=1 / k_{0}(b, \eta)$ where $k_{0}$ is defined as in Theorem 7.1. Let $\mathscr{A}$ be an $\ell$-augmented weighted 3-graph with $t$ vertices, and let $\psi_{\mathscr{A}}^{*}$ be a maximum fractional $\mathscr{F}_{0}$-packing of $\mathscr{A}$. We define an auxiliary $b$-uniform vertex-weighted hypergraph $\mathscr{K}$ as follows. The vertex set of $\mathscr{K}$ is $V(\mathscr{K})=\left\{v_{e}: e \in E(\mathscr{A})\right\}$, where the weight $w\left(v_{e}\right)$ of a vertex $v_{e}$ is the weight in $\mathscr{A}$ of the corresponding triple $e$. Then note that $m=|V(\mathscr{K})|=|E(\mathscr{A})| \leq \ell^{3} t^{3}$. A set of $b$ vertices of $\mathscr{K}$ form an edge of $\mathscr{K}$ if and only if the corresponding $b$ triples of $\mathscr{A}$ form a copy of $\mathscr{F}_{0}$ in $\mathscr{A}$. Then $\psi_{\mathscr{A}}^{*}$ corresponds to a fractional packing $\phi^{*}$ of $\mathscr{K}$, such that $\left|\phi^{*}\right|$ $=\nu_{\mathscr{F}_{0}}^{*}(\mathscr{A})$.

Since Theorem 7.1 applies only to fractional packings that are "uniformly small," we first modify $\mathscr{K}$ by removing edges $E$ for which $\phi^{*}(E) \geq \tau$ (we will replace these edges later). Let $\mathscr{E}_{0}=\left\{E \in \mathscr{K}: \phi^{*}(E) \geq \tau\right\}$. Then we define a new vertex-weighted hypergraph $\mathcal{K}^{\prime}$, where

(i) $V\left(\mathscr{K}^{\prime}\right)=V(\mathscr{K})$,

(ii) $w_{\mathscr{K}^{\prime}}(v)=w_{\mathscr{K}}(v)-\sum_{E \ni v, E \in \mathscr{E}_{0}} \phi^{*}(E)$ for each $v \in V\left(\mathscr{K}^{\prime}\right)$,

(iii) $\mathscr{K}^{\prime}=\mathscr{K} \backslash \mathscr{E}_{0}$.

We also define a fractional packing $\phi^{\prime}$ on $\mathscr{K}^{\prime}$ by $\phi^{\prime}(E)=\phi^{*}(E)$ for each $E \in \mathscr{K}^{\prime}$. Then

$$
\left|\phi^{\prime}\right|=\left|\phi^{*}\right|-\sum_{E \in \mathscr{E}_{0}} \phi^{*}(E) .
$$

Note then that $\phi^{\prime}$ is in fact a fractional packing since for $v \in V\left(\mathscr{K}^{\prime}\right)$ we have

$$
\sum_{E \ni v} \phi^{\prime}(E)=\sum_{E \ni v, E \in \mathscr{K}} \phi^{*}(E)-\sum_{E \ni v, E \in \mathscr{E}_{0}} \phi^{*}(E) \leq w_{\mathscr{K}}(v)-\sum_{E \ni v, E \in \mathscr{E}_{0}} \phi^{*}(E)=w_{\mathscr{K}^{\prime}}(v) .
$$


Also, for every $E \in \mathscr{K}^{\prime}$ we have $\phi^{\prime}(E)<\tau$. Then since $\tau=1 / k_{0}(b, \eta)$, by Theorem 7.1 there exists a $\tau$-bounded fractional packing $\bar{\phi}$ of $\mathscr{K}^{\prime}$ such that $|\bar{\phi}| \geq\left|\phi^{\prime}\right|-\eta m$. We therefore define a fractional packing $\phi$ of $\mathscr{K}$ as follows: We let

$$
\phi(E)= \begin{cases}\phi^{*}(E) & \text { if } E \in \mathscr{E}_{0} \\ \bar{\phi}(E) & \text { if } E \in \mathscr{K}^{\prime}\end{cases}
$$

Then $\phi$ is $\tau$-bounded by construction. Also, by (9),

$$
|\phi|=\sum_{E \in \mathscr{E}_{0}} \phi^{*}(E)+|\bar{\phi}| \geq \sum_{E \in \mathscr{E}_{0}} \phi^{*}(E)+\left|\phi^{\prime}\right|-\eta m=\left|\phi^{*}\right|-\eta m \geq \nu_{\mathscr{F}_{0}}^{*}(\mathscr{A})-\eta \ell^{3} t^{3} .
$$

Since $\phi$ corresponds to a fractional $\mathscr{F}_{0}$-packing $\phi_{\mathscr{A}}$ of $\mathscr{A}$, the result follows.

\section{PROOF OF LEMMA 3.4}

In order to prove Lemma 3.4, we shall make use of the following two theorems. The first is a special case of a theorem of Kahn [11], which generalizes a theorem of Frankl and Rödl [5] on packings in hypergraphs. By a packing of a hypergraph $\mathscr{K}$ we mean a set of pairwise disjoint edges of $\mathscr{K}$.

Theorem 8.1. Let an integer $b$ and a real number $\lambda>0$ be given. Then there exist $\beta=$ $\beta(b, \lambda)>0, D_{0}=D_{0}(b, \lambda)$ and $n_{0}=n_{0}(b, \lambda)$ such that the following holds. Let $\mathcal{K}$ be a $b$-uniform hypergraph with vertex set $X,|X| \geq n_{0}$, such that for some $D \geq D_{0}$

(i) $\operatorname{deg}_{\%}(x) \leq D$ for all vertices $x \in X$,

(ii) $\operatorname{deg}_{\mathscr{K}}(x, y)<\beta D$ for all pairs of distinct vertices $x, y \in X$.

Then $\mathscr{K}$ has a packing of size at least $(1-\lambda)|\mathscr{K}| / D$.

Our approach to proving Lemma 3.4 will be as follows. We will focus on a subhypergraph $\mathscr{H}^{\prime} \subset \mathscr{H}$, and a special set $\mathscr{Y}_{\mathscr{F}_{0}}\left(\mathscr{H}^{\prime}\right)$ of copies of $\mathscr{J}_{0}$ in $\mathscr{H}^{\prime}$, each of which is partite-isomorphic to $\mathscr{F}_{0}$ in $\mathscr{H}$. The definitions of $\mathscr{H}^{\prime}$ and $\mathscr{Y}_{\mathscr{F}_{0}}\left(\mathscr{H}^{\prime}\right)$ appear in Section 10. We will apply Theorem 8.1 to the hypergraph whose vertex set is $\mathscr{H}^{\prime}$, and whose edges are the sets of triples which form copies of $\mathscr{F}_{0}$ in $\mathscr{Y}_{\mathscr{F}_{0}}\left(\mathscr{H}^{\prime}\right)$, to show that $\mathscr{H}^{\prime}$ contains a packing of copies of $\mathscr{F}_{0}$ that covers all but at most $\lambda|\mathscr{H}|$ triples of $\mathscr{H}$. We describe the properties of $\mathscr{H}^{\prime}$ in the following lemma, the proof of which depends on the Extension Theorem and is found in Section 10.

Lemma 8.2. Let $\mathscr{F}_{0}$ be a 3-uniform hypergraph with vertex set $[k]$ and with $b$ triples. Let real numbers $\lambda>0$ and $\alpha>0$ be given. Then there exists $\zeta=\zeta\left(\mathscr{\Phi}_{0}, \lambda, \alpha\right)$ such that, for all integers $\ell>1 / \zeta$, there exist $r=r\left(\mathscr{F}_{0}, \lambda, \alpha, \ell\right), \epsilon=\epsilon\left(\mathscr{F}_{0}, \lambda, \alpha, \ell\right)$, and $n_{1}=n_{1}\left(\mathscr{F}_{0}\right.$, $\lambda, \alpha, \ell)$ such that the following holds. Let $\mathscr{H}$ and $G$ be such that

(i) He is a k-partite 3-cylinder with $k$-partition $V_{1}, \ldots, V_{k},\left|V_{1}\right|=\cdots=\left|V_{k}\right|=n$, where $n \geq n_{1}$, 
(ii) $G=\bigcup_{1 \leq i<j \leq k} G^{i j}$ is an underlying $(\ell, \epsilon, k)$-cylinder,

(iii) for all triples $\{i, j, s\} \in \mathscr{F}_{0}, \mathscr{H}\left(G^{i j}, G^{i s}, G^{j s}\right)$ is $(\alpha, \zeta, r)$-regular with respect to $\left(G^{i j}, G^{i s}, G^{j s}\right)$.

Then there exists a subhypergraph $\mathscr{H}^{\prime}$ of $\mathscr{H}$ and a set $\mathscr{S}_{\mathscr{F}_{0}}\left(\mathcal{H}^{\prime}\right)$ of copies of $\mathscr{F}_{0}$ in $\mathscr{H}^{\prime}$, each of which is partite-isomorphic to $\mathscr{F}_{0}$ in $\mathscr{H}$, with the following properties:

(a) For every triple e of $\mathscr{H}^{\prime}$, the number $q_{\mathscr{F}_{0}}\left(\right.$ e) of elements of $\mathscr{Y}_{\mathscr{F}_{0}}\left(\mathscr{H}^{\prime}\right)$ that contain e satisfies

$$
q_{\Phi_{0}}(e) \leq(1+\lambda) \frac{\alpha^{b-1}}{\ell^{\left(\begin{array}{l}
k \\
2
\end{array}\right)-3}} n^{k-3} \text {. }
$$

(b) The set $\mathscr{G}_{\mathscr{F}_{0}}\left(\mathscr{H}^{\prime}\right)$ satisfies

$$
\left|\mathscr{S}_{\mathscr{F}_{0}}\left(\mathscr{H}^{\prime}\right)\right| \geq(1-\lambda) \frac{\alpha^{b}}{\ell^{\left(\begin{array}{l}
k \\
2
\end{array}\right)}} n^{k}
$$

We are now ready to prove Lemma 3.4.

Proof of Lemma 3.4. Let $\mathscr{F}_{0}, \lambda$, and $\alpha$ be given, and let $b$ denote the number of triples of $\mathscr{F}_{0}$. We then define $\theta=\min \left\{\zeta\left(\mathscr{F}_{0}, \lambda / 8, \alpha\right),(1-\lambda / 2)(1-\lambda)^{-1}-1\right\}$. To define $r_{0}, \epsilon_{0}$, and $m_{0}$, let $\ell>1 / \theta$ be given. Let $r_{0}=r\left(\mathscr{F}_{0}, \lambda / 8, \alpha, \ell\right)$ and $\epsilon_{0}=\epsilon\left(\mathscr{F}_{0}, \lambda / 8, \alpha, \ell\right)$, where the functions $r$ and $\epsilon$ are as defined in Lemma 8.2. We also set

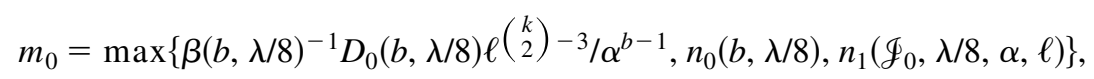

where the functions $\beta, n_{0}$, and $D_{0}$ are as defined in Theorem 8.1, and $n_{1}$ as in Lemma 8.2.

Suppose $\mathscr{H}$ and $G$ satisfy the conditions (i)-(iii) in the statement of Lemma 3.4, where $\left|V_{1}\right|=\cdots=\left|V_{k}\right|=n \geq m_{0}$. Then, by definition of $r_{0}, \epsilon_{0}$, and $m_{0}$, $\mathscr{H}$ and $G$ satisfy the conditions (i)-(iii) of Lemma 8.2 with parameters $\mathscr{F}_{0}, \lambda / 8, \alpha$, and $\ell$. Therefore, by Lemma 8.2, there exists a subhypergraph $\mathscr{H}^{\prime}$ of $\mathscr{H}$ and a set $\mathscr{S}_{\mathscr{F}_{0}}\left(\mathscr{H}^{\prime}\right)$ of copies of $\mathscr{F}_{0}$ in $\mathscr{H}^{\prime}$ that satisfy Properties (a) and (b).

Now we let the $b$-uniform hypergraph $\mathscr{K}$ be defined as follows. The vertex set $V(\mathscr{K})=$ $\mathscr{H}^{\prime}$, and a set of $b$ triples of $\mathscr{H}^{\prime}$ forms an edge of $\mathscr{K}$ if and only if it is the triple set of a subhypergraph of $\mathscr{H}^{\prime}$ that is an element of $\mathscr{S}_{\mathscr{F}_{0}}\left(\mathscr{H}^{\prime}\right)$. Then by Lemma 8.2(a) we have that $\operatorname{deg}_{\mathscr{K}}(e)=q_{\mathscr{F}_{0}}(e)$ satisfies Condition (i) in Theorem 8.1 for every vertex $e$ of $\mathscr{K}$, where $D=(1+\lambda / 8) \frac{\alpha^{b-1}}{\ell\left(\begin{array}{c}k \\ 2\end{array}\right)-3} n^{k-3}$. Moreover, we note that for any two distinct $e_{1}, e_{2} \in V(\mathscr{K})$ we have $\operatorname{deg}_{\mathscr{K}}\left(e_{1}, e_{2}\right)<n^{k-4}$, since two distinct triples fix at least 4 vertices in $\mathscr{H}$. Now $m_{0}$ is large enough such that $n^{k-4}<\beta(b, \lambda / 8) D$ for all $n \geq m_{0}$, and also such that $D$ $>\frac{\alpha^{b-1}}{\ell\left(\begin{array}{c}k \\ 2\end{array}\right)-3} n^{k-3} \geq D_{0}(b, \lambda / 8)$, and certainly $|V(\mathscr{K})|=|\mathscr{H}|>n \geq n_{0}(b, \lambda / 8)$. Therefore, by Theorem 8.1 we have that $\mathscr{K}$ contains a packing $\mathscr{T}$ of size at least $(1-\lambda / 8)|\mathscr{K}| / D$. Then using Lemma 8.2(b) we find

$$
|\mathscr{T}| \geq(1-\lambda / 8)\left|\mathscr{S}_{\mathscr{F}_{0}}\left(\mathscr{H}^{\prime}\right)\right| / D \geq \frac{(1-\lambda / 8)^{2}}{(1+\lambda / 8)} \frac{\alpha^{b} n^{k}}{\ell^{(k}\left(\begin{array}{l}
k \\
2
\end{array}\right)} \frac{\ell^{\left(\begin{array}{c}
k \\
2
\end{array}\right)-3}}{\alpha^{b-1} n^{k-3}} \geq(1-\lambda / 2) \frac{\alpha n^{3}}{\ell^{3}} .
$$


In other words, the packing $\mathscr{T}$ gives a family of triple-disjoint sub-hypergraphs of $\mathscr{H}^{\prime}$ (and hence of $\mathscr{H}$ ), each partite-isomorphic to $\mathscr{F}_{0}$ in $\mathscr{H}$, of size at least $(1-\lambda / 2) \frac{\alpha n^{3}}{\ell^{3}}$. But by definition of $(\alpha, \theta, r)$-regularity, each $\mathscr{H}\left(G^{i j}, G^{i s}, G^{j s}\right)$ has at most $(1+\theta) \alpha n^{3} / \ell^{3}$ triples. Since each copy of $\mathscr{F}_{0}$ in the packing $\mathscr{T}$ has precisely one triple in each $\mathscr{H}\left(G^{i j}, G^{i s}\right.$, $\left.G^{j s}\right)$, we conclude that $\mathscr{T}$ covers at least a $(1-\lambda / 2)(1+\theta)^{-1}>(1-\lambda)$ proportion of the triples in each $\mathscr{H}\left(G^{i j}, G^{i s}, G^{j s}\right)$, and hence it covers all but $\lambda|\mathscr{H}|$ triples of $\mathscr{H}$.

\section{PROOF OF LEMMA 3.10}

First we prove (i). We obtain $\mathscr{H}^{\prime}$ from $\mathscr{H}$ by removing the following triples of $\mathscr{H}$ :

(a) the triples of $\mathscr{H}$ that have more than one vertex in some vertex class $V_{i}$ of $\mathscr{P}$,

(b) the triples that have a vertex in the vertex class $V_{0}$,

(c) triples that contain a vertex of $V_{i}$ and a vertex of $V_{j}$ such that the pair $i, j$ does not satisfy Condition (iii) of Definition 2.4,

(d) triples on underlying bipartite graphs $P_{0}^{i j}$, (where $i, j$ satisfies Condition (iii) of Definition 2.4)

(e) triples that lie in $(\delta, r)$-irregular 3-partite 3-cylinders of $\mathscr{H}$.

For each of these cases, we estimate the number of triples of $\mathscr{H}$ that must be removed, using the bounds given in Definition 2.4.

(a) For each $i$ we have $\left|V_{i}\right|=m \leq n / t$ so the total number of such triples is at most $t(n / t)^{2} n=n^{3} / t \leq n^{3} / t_{0} \leq \eta n^{3} / 100$, where we use the fact that $n \geq N_{1}$ and the definition of $N_{1}$ and of $t_{0}$ (see Definition 3.7).

(b) Since $\left|V_{0}\right| \leq t \leq T_{0}$, we have at most $T_{0} n^{2} \leq n^{3}\left(T_{0} / n\right)<\eta n^{3} / 100$ such triples, again using the definition of $N_{1}$.

(c) Since there are at most $\gamma\left({ }_{2}^{t}\right)$ pairs $i, j$ that do not satisfy Condition (iii) of Definition 2.4 , the number of such triples is at most $\gamma\left({ }_{2}^{t}\right) m^{2} n<\gamma(t m)^{2} n / 2<\gamma n^{3} / 2<$ $\eta n^{3} / 200$, where here we use the definition of $\gamma$ [see Definition 3.7 (iv)].

(d) For each such $i, j$ we know that $\left|P_{0}^{i j}\right| \leq \gamma m^{2}$, so the total number of such triples is at most $\left(\begin{array}{c}t \\ 2\end{array}\right) \gamma m^{2} n \leq \gamma n^{3} / 2<\eta n^{3} / 200$, where again we use the definition of $\gamma$.

(e) The number of such triples is at most $\delta n^{3} \leq \alpha \eta n^{3} / 100<\eta n^{3} / 100$.

Therefore, the total number of triples of $\mathscr{H}$ that are removed to form $\mathscr{H}^{\prime}$ is at most $4 \eta n^{3} / 100$ as required.

Now we consider (ii). Given the maximum fractional packing $\psi$ of $\mathscr{H}$, we first define a function $\psi^{\prime}:\left(\begin{array}{c}\mathscr{K}^{\prime} \\ \mathscr{F}_{0}\end{array}\right) \rightarrow[0,1]$ by $\psi^{\prime}(\mathscr{F})=\psi(\mathscr{F})$ for each $\mathscr{F} \in\left(\begin{array}{l}\mathscr{F} \mathcal{F}_{0}^{\prime} \\ )\end{array}\right)$. Then $\psi^{\prime}$ is a fractional packing of $\mathscr{H}^{\prime}$, and $\left|\psi^{\prime}\right| \geq|\psi|-\left(|\mathscr{H}|-\left|\mathscr{H}^{\prime}\right|\right)$, since each triple $e$ of $\mathscr{H}$ satisfies $\sum_{\mathscr{F} \in(}\left(\begin{array}{l}\mathscr{H} \\ \mathscr{F}_{0}\end{array}\right): \mathscr{g} \ni e \psi(\mathscr{F}) \leq 1$. Therefore, by (i),

$$
\left|\psi^{\prime}\right| \geq \nu_{\mathscr{F}_{0}}^{*}(\mathscr{H})-\eta n^{3} / 25
$$

Now we use the fractional packing $\psi^{\prime}$ of $\mathscr{H}^{\prime}$ to construct a fractional packing $\psi_{\mathscr{A}}$ of $\mathscr{A}$ with $\left|\psi_{\mathscr{A}}\right| \geq(\ell / m)^{3}\left|\psi^{\prime}\right|$. First, for $\mathscr{\mathscr { F }} \in \mathscr{C}\left(\mathscr{H}^{\prime}, \mathscr{P}, \mathscr{F}_{0}\right)$ (i.e., copies of $\mathscr{E}_{0}$ that are crossing in $\mathscr{P}$ ) we define the projection $\pi(\mathscr{F}) \in\left(\mathscr{\mathscr { F }}_{0}\right)$ as follows. Let the vertex set of $\mathscr{\mathscr { F }}$ be $\left\{w_{1}, \ldots\right.$, 
$\left.w_{k}\right\}$, where $k=\left|V\left(\mathscr{F}_{0}\right)\right|$ and $w_{u} \in V_{i_{u}}$ for $1 \leq u \leq k$. Then by definition of $\mathscr{H}^{\prime}$, each triple $e$ of $\mathscr{F}$ lies in a 3-partite 3-cylinder $\mathscr{H}^{\prime}\left(P_{e}\right)$ of $\mathscr{H}^{\prime}$ which is $(\delta, r)$-regular with respect to its underlying triad $P_{e}$. We then let $\pi(\mathscr{F})$ be the copy $\mathscr{J}^{\prime}$ of $\mathscr{F}_{0}$ in $\mathscr{A}$ with vertex set $\left\{v_{i_{1}}, \ldots, v_{i_{k}}\right\}$, and where $\left\{c_{\beta_{u x}}^{v_{i_{u}} v_{i_{x}}}, c_{\beta_{u y}}^{v_{i_{u}} v_{i_{x}}}, c_{\beta_{x y}}^{v_{i_{u}} v_{i x}}\right\}$ is a triple of $\mathscr{g}^{\prime}$ precisely when $\left(P_{\beta_{u x}}^{v_{i_{u}} v_{i_{x}}}\right.$, $\left.P_{\beta_{u y}}^{v_{i u} v_{i x}}, P_{\beta_{x y}}^{v_{i u} v_{i x}}\right)=P_{e}$ for some triple $e$ of $\mathscr{F}_{0}$. [Note that this uniquely determines the pair set $C\left(\mathscr{F}^{\prime}\right)$ also.]

Given $\psi^{\prime}$, we define $\psi_{\mathscr{A}}:\left(\underset{\mathscr{F}_{0}}{\mathscr{A}}\right) \rightarrow[0,1]$ as follows. Let $\mathscr{J}^{\prime} \in\left(\begin{array}{c}\mathscr{A} \\ \mathscr{F}_{0}\end{array}\right)$ be given as in Definition 3.2. Then we let

$$
\psi_{\mathscr{A}}\left(\mathscr{F}^{\prime}\right)=(1+\eta / 100)^{-1}(\ell / m)^{3} \sum\left\{\psi^{\prime}(\mathscr{F}): \mathscr{\mathscr { F }} \in \mathscr{C}\left(\mathscr{H}^{\prime}, \mathscr{P}, \mathscr{F}_{0}\right), \pi(\mathscr{F})=\mathscr{F}^{\prime}\right\} .
$$

To see that $\psi_{\mathscr{A}}$ is a fractional packing, we fix a triple $e=\left\{c_{\beta_{1}}^{v_{i} v_{s}}, c_{\beta_{2}}^{v_{i} v_{h}}, c_{\beta_{3}}^{v_{h} v_{s}}\right\}$ of $\mathscr{A}$. Let us denote by $P_{e}=\left(P_{\beta_{1}}^{v_{i} v_{s}}, P_{\beta_{2}}^{v_{i} v_{h}}, P_{\beta_{3}}^{v_{h} v_{s}}\right)$ the corresponding triad. Then we have

$$
\begin{aligned}
\sum_{\mathscr{F}^{\prime} \ni e} \psi_{\mathscr{A}}\left(\mathscr{F}^{\prime}\right) & =(1+\eta / 100)^{-1}(\ell / m)^{3} \sum_{\mathscr{F}^{\prime} \ni e} \sum\left\{\psi^{\prime}(\mathscr{F}): \mathscr{F} \in \mathscr{C}\left(\mathscr{H}^{\prime}, \mathscr{P}, \mathscr{F}_{0}\right), \pi(\mathscr{F})=\mathscr{F}^{\prime}\right\} \\
& =(1+\eta / 100)^{-1}(\ell / m)^{3} \sum_{T \in \mathscr{H}^{\prime}\left(P_{e}\right)} \sum_{\mathscr{F} \in \mathscr{C}\left(\mathscr{H}^{\prime}, \mathscr{P}, \mathscr{F}_{0}\right), \mathscr{F} \ni T} \psi^{\prime}(\mathscr{\mathscr { S }}) \\
& \leq(1+\eta / 100)^{-1}(\ell / m)^{3} \sum_{T \in \mathscr{H}^{\prime}\left(P_{e}\right)} 1=\omega(e),
\end{aligned}
$$

where the last line follows from the definition of $\mathscr{A}$ (see Definition 3.9) and Fact 2.3. Therefore, $\psi_{\mathscr{A}}$ is a fractional packing of $\mathscr{A}$. Moreover, we have

$$
\begin{aligned}
\left|\psi_{\mathscr{A}}\right| & =\sum_{\mathscr{F}^{\prime} \in\left(\begin{array}{l}
\mathscr{A} \\
\mathscr{F}_{0}
\end{array}\right)} \psi_{\mathscr{A}}\left(\mathscr{F}^{\prime}\right) \\
& =(1+\eta / 100)^{-1}(\ell / m)^{3} \sum_{\mathscr{F}^{\prime} \in\left(\begin{array}{l}
\mathscr{A} \\
\mathscr{F}_{0}
\end{array}\right)} \sum\left\{\psi^{\prime}(\mathscr{F}): \mathscr{F} \in \mathscr{C}\left(\mathscr{H}^{\prime}, \mathscr{P}, \mathscr{I}_{0}\right), \pi(\mathscr{F})=\mathscr{F}^{\prime}\right\} \\
& =(1+\eta / 100)^{-1}(\ell / m)^{3} \sum\left\{\psi^{\prime}(\mathscr{F}): \mathscr{F} \in \mathscr{C}\left(\mathscr{H}^{\prime}, \mathscr{P}, \mathscr{F}_{0}\right)\right\} .
\end{aligned}
$$

Now since $\mathscr{P}$ respects the initial partition 2 , by (3) we find

$$
\begin{aligned}
\left|\psi_{\mathscr{A}}\right| & \geq(1+\eta / 100)^{-1}(\ell / m)^{3}\left(\left|\psi^{\prime}\right|-\sum\left\{\psi^{\prime}(\mathscr{\mathscr { F }}): \mathscr{\mathscr { E }} \in\left(\begin{array}{c}
\mathscr{H}^{\prime} \\
\mathscr{F}_{0}
\end{array}\right), \mathscr{\mathscr { E }} \text { not crossing }\right\}\right) \\
& \geq(1+\eta / 100)^{-1}(\ell / m)^{3}\left(\left|\psi^{\prime}\right|-\eta \nu_{\mathscr{F}_{0}}^{*}(\mathscr{H}) / 100\right) .
\end{aligned}
$$

Now, using (10), we find

$$
\begin{aligned}
(m / \ell)^{3}\left|\psi_{\mathscr{A}}\right| & \geq(1-\eta / 100)\left(\left|\psi^{\prime}\right|-\eta \nu_{\mathscr{F}_{0}}^{*}(\mathscr{H}) / 100\right) \\
& \geq(1-\eta / 100)\left(\nu_{\mathscr{F}_{0}}^{*}(\mathscr{H})-\eta n^{3} / 25-\eta n^{3} / 100\right) \\
& \geq \nu_{\mathscr{F}_{0}}^{*}(\mathscr{H})-3 \eta n^{3} / 50,
\end{aligned}
$$

since $\nu_{\mathscr{F}_{0}}^{*}(\mathscr{H})<n^{3}$. Then since $\left|\psi_{\mathscr{A}}\right| \leq \nu_{\mathfrak{F}_{0}}^{*}(\mathscr{A})$, the result follows. 


\section{THE EXTENSION THEOREM}

In this section we present the already advertised Extension Theorem. Our proof of this result is quite complicated, so we divide the proof across the next three sections. In this section, our goal is to state and discuss the Extension Theorem and related results.

In Section 10.1, we provide definitions and facts extending some notions given in Section 2. In Section 10.2, we state the Extension Theorem and discuss how its proof is given in later sections. In Section 10.3, we give the proof of Lemma 8.2, the application for which the Extension Theorem is required. In Section 10.4, we give the proof of the upcoming Corollary 10.10, a tool needed in our proof of Lemma 8.2.

\subsection{Background Concepts}

In this subsection, we provide introductory background definitions, notation and facts. We begin with the following simple fact about $(\ell, \epsilon, k)$-cylinders. In what follows, when $G=\bigcup_{1 \leq i<j \leq k} G^{i j}$ is $k$-partite cylinder with $k$-partition $\left(V_{1}, \ldots, V_{k}\right)$ and $j \in[k]$, we write the $j$-neighbors of $v$ as $N_{j}(v)=\left\{w \in V_{j}:\{v, w\} \in G\right\}$.

Fact 10.1. Suppose $G=\bigcup_{1 \leq i<j \leq k} G^{i j}$ is an $(\ell, \epsilon, k)$-cylinder with k-partition $\left(V_{1}, \ldots, V_{k}\right),\left|V_{1}\right|=\cdots=\left|V_{k}\right|=n$. Fix $i \in[k]$. All but $2 k \epsilon n$ vertices $v_{i} \in V_{i}$ satisfy that for all $j \in[k], j \neq i$,

$$
\frac{n}{\ell}(1-\epsilon)<\left|N_{j}\left(v_{i}\right)\right|<\frac{n}{\ell}(1+\epsilon) .
$$

We remark here that sometimes we consider the situation when two $k$-partite cylinders $G$ and $F$ are simultaneously defined on the same vertex set $V$ with $k$-partition $\left(V_{1}, \ldots, V_{k}\right)$. In such situations, we will still want to denote the $j$-neighbors of each cylinder. We denote by $N_{G, j}(v)$ the $j$-neighborhood of the vertex $v$ in the cylinder $G$, that is,

$$
N_{G, j}(v)=\left\{w \in V_{j}:\{v, w\} \in G\right\} .
$$

Similarly,

$$
N_{F, j}(v)=\left\{w \in V_{j}:\{v, w\} \in F\right\} .
$$

To extend the notation in (11) and (12) from a vertex $v$ to an edge $e=\left\{v_{1}, v_{2}\right\}$, we write for an integer $i \in\{3, \ldots, k\}$

$$
N_{G, i}(e)=N_{G, i}\left(v_{1}\right) \cap N_{G, i}\left(v_{2}\right) .
$$

and

$$
N_{F, i}(e)=N_{F, i}\left(v_{1}\right) \cap N_{F, i}\left(v_{2}\right) .
$$


We extend our definition of $\mathscr{K}_{3}(G)$ by defining the following auxiliary set system pertaining to a $k$-partite cylinder $G$. In what follows, by a clique in a graph $G$, we mean a complete subgraph $G^{\prime} \subseteq G$.

Definition 10.2. For a $k$-partite cylinder $G$, denote by $\mathscr{K}_{j}(G), 1 \leq j \leq k$, that j-uniform hypergraph whose edges are precisely those j-element subsets of $V(G)$ which span cliques of order $j$ in $G$.

Observe that the quantity $\left|\mathscr{K}_{j}(G)\right|$ counts the total number of cliques in $G$ of order $j$, that is, $\left|\mathscr{K}_{j}(G)\right|=\left|\left\{X \subseteq V(G):|X|=j,[X]^{2} \subseteq G\right\}\right|$. For an $(\ell, \epsilon, k)$-cylinder $G$, the quantity $\left|\mathscr{K}_{k}(G)\right|$ is easy to estimate, as the following fact shows.

Fact 10.3. For any positive integers $k, \ell$ and positive real $\theta$, there exists $\epsilon$ so that whenever $G$ is an $(\ell, \epsilon, k)$-cylinder with $k$-partition $\left(V_{1}, \ldots, V_{k}\right),\left|V_{1}\right|=\cdots=\left|V_{k}\right|=n$, then

$$
(1-\theta) \frac{n^{k}}{\ell^{\left(\begin{array}{l}
k \\
2
\end{array}\right)}}<\left|\mathscr{K}_{k}(G)\right|<(1+\theta) \frac{n^{k}}{\ell^{\left(\begin{array}{l}
k \\
2
\end{array}\right)}} .
$$

Note that Fact 10.3 extends Fact 2.3. It can be proved using a standard argument from the definition of an $(\ell, \epsilon, k)$-cylinder. As Fact 10.3 includes Fact 2.3 as a special case (i.e., when $k=3$ ), we from this point on only refer to Fact 10.3 .

The following definition gives a slight variation on Definition 10.2. While we only directly use this definition a bit later, we introduce it to motivate topics soon visited.

Definition 10.4. For a k-partite cylinder $G$ and for $\{x, y, z\} \in \mathscr{K}_{3}(G)$, set

$$
\mathscr{E}_{K_{j}^{(2)}}^{G}(\{x, y, z\})=\left\{Y \in \mathscr{K}_{j}(G):\{x, y, z\} \in[Y]^{3}\right\} .
$$

In other words, $\mathscr{E}_{K_{j}^{(2)}}^{G}(\{x, y, z\})$ is the set of all cliques in $G$ of order $j$ which contain $\{x, y$, z\} as a triangle.

Observe that $\left|\mathscr{E}_{K_{j}^{(2)}}^{G}(\{x, y, z\})\right|$ counts the number of extensions of the triangle $\{x, y, z\}$ to $j$-cliques in $G$. For an $(\ell, \epsilon, k)$-cylinder $G$, the quantity $\left|\mathscr{E}_{K_{k}^{(2)}}^{G}(\{x, y, z\})\right|$ is easy to estimate. The following fact is a simple variation on Fact 10.3 .

Fact 10.5. For any positive integers $k, \ell$ and positive real $\theta$, there exists $\epsilon$ so that whenever $G=\bigcup_{1 \leq i<j \leq k} G^{i j}$ is an $(\ell, \epsilon, k)$-cylinder with $k$-partition $\left(V_{1}, \ldots, V_{k}\right),\left|V_{1}\right|$ $=\cdots=\left|V_{k}\right|=n$, then all but $6 k \epsilon n^{3}$ triangles $\{x, y, z\} \in \mathcal{K}_{3}\left(G^{12} \cup G^{13} \cup G^{23}\right)$ satisfy

$$
(1-\theta) \frac{n^{k-3}}{\ell^{\left(\begin{array}{l}
k \\
2
\end{array}\right)-3}}<\left|\mathscr{E}_{K_{k}^{(2)} G}^{G}(\{x, y, z\})\right|<(1+\theta) \frac{n^{k-3}}{\ell^{\left(\begin{array}{l}
k \\
2
\end{array}\right)-3}} .
$$

As with Fact 10.3, the proof of Fact 10.5 follows along standard lines from the definition of an $(\ell, \epsilon, k)$-cylinder.

We now give definitions pertaining to 3-uniform hypergraphs. Recall that in Section 2 
we were interested in 3-partite 3-cylinders arising from an $(\ell, t, \gamma, \epsilon)$-partition $\mathscr{P}$ of a 3-uniform hypergraph $\mathcal{H}$. In this situation, each pair $\left(V_{i}, V_{j}\right)$ of vertex classes of the partition $\mathscr{P}$ was joined by many edge-disjoint bipartite graphs $P_{\beta}^{i j}$, so to specify a 3-partite 3 -cylinder of $\mathscr{H}$ we used the notation $\mathscr{H}\left(P_{\beta}^{i j}, P_{\rho}^{i s}, P_{\mu}^{j s}\right)$ to denote the 3-partite 3-cylinder of $\mathscr{H}$ on the triad $P=\left(P_{\beta}^{i j}, P_{\rho}^{i s}, P_{\mu}^{j s}\right)$ of $\mathscr{P}$. However, in this section we will be concentrating exclusively on 3-uniform hypergraphs whose underlying substructure is one fixed $(\ell, \epsilon, k)$-cylinder $G$ with vertex partition $V_{1}, \ldots, V_{k}$, that is, each pair $\left(V_{i}, V_{j}\right)$ of vertex classes will be joined by precisely one bipartite graph $G^{i j}$. Here then, to specify a particular induced 3-partite 3-cylinder, we need only specify the vertex classes, so we will write $\mathcal{H}\left(\left\{V_{i}, V_{j}, V_{s}\right\}\right)$ instead of $\mathcal{H}\left(G^{i j}, G^{i s}, G^{j s}\right)$. In fact we make the following more general definition.

Definition 10.6. Recall that any k-partite, 3-uniform hypergraph $\mathscr{H}$ with a fixed $k$ partition $\left(V_{1}, \ldots, V_{k}\right)$ is referred to as a $k$-partite 3-cylinder. For $B \subseteq[k]$, we define the B-3-cylinder of $\mathscr{H}$ as that subhypergraph $\mathscr{H}(B)$ of $\mathscr{H}$ induced on $\bigcup_{i \in B} V_{i}$.

As in Definition 10.2, we define an auxiliary set system pertaining to a 3-cylinder $\mathscr{H}$. In what follows, by a clique in a 3 -uniform hypergraph $\mathscr{H}$, we mean a complete 3 -uniform subhypergraph $\mathscr{H}^{\prime} \subseteq \mathscr{H}$.

Definition 10.7. For a $k$-partite 3 -cylinder $\mathscr{H}$, denote by $\mathscr{K}_{j}(\mathscr{H}), 1 \leq j \leq k$, that $j$-uniform hypergraph whose edges are precisely those j-element subsets of $V(\mathcal{H})$ which span a

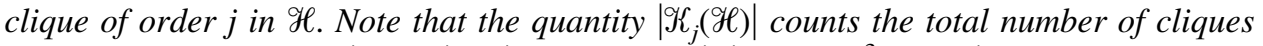
in $\mathscr{H}$ of order $j$, that is, $\left|\mathscr{K}_{j}(\mathscr{H})\right|=\left|\left\{X \subseteq V(\mathscr{H}):|X|=j,[X]^{3} \subseteq \mathscr{H}\right\}\right|$.

We remark that a formula for computing $\left|\mathscr{K}_{k}(\mathscr{H})\right|$ in certain contexts, analogous to Fact 10.3 , is given later in the upcoming Theorem 10.8 in the next subsection.

\subsection{The Extension Theorem}

We begin this subsection by formulating the following environment which will be the setting for much of our work. Because we consider other environments later, we call the following Setup 1. For a given integer $k \geq 3$, 3-uniform hypergraph $\mathscr{f}_{0} \subseteq[k]^{3}, \alpha, \delta$ positive reals, $\ell, r$ positive integers, $\epsilon>0$ and $n$ a positive integer, consider the following.

\section{Setup 1:}

(1) $\mathcal{H}$ is a $k$-partite 3 -cylinder with $k$-partition $\left(V_{1}, \ldots, V_{k}\right),\left|V_{1}\right|=\cdots=\left|V_{k}\right|=n$.

(2) $G=\bigcup_{1 \leq i<j \leq k} G^{i j}$ is an underlying $(\ell, \epsilon, k)$-cylinder.

(3) For all $B \in \mathscr{F}_{0}, \mathscr{H}(B)$ is $(\alpha, \delta, r)$-regular with respect to $G(B)$; otherwise, for every $B \in[k]^{3} \backslash \mathscr{E}_{0}, \mathcal{H}(B)=\varnothing$.

To help motivate our main result, The Extension Theorem, we first consider the following related theorem proved in [12] and [13].

Theorem 10.8 (Counting Lemma). For all integers $k \geq 4$, for all $\alpha, \beta>0$, there exists a constant $\delta>0$ so that, for all integers $\ell \geq \frac{1}{\delta}$, there exists an integer $r$ and $\epsilon>0$ so that for $n$ sufficiently large, whenever k-partite 3-cylinder $\mathscr{H}$ and $G=\bigcup_{1 \leq i<j \leq k} G^{i j}$ satisfy 
the conditions of Setup 1 with constants $k, \alpha, \delta, \ell, r, \epsilon$ and $n$, and 3-uniform hypergraph $\mathscr{f}_{0}=K_{k}^{(3)}$, then

$$
\frac{\alpha^{\left(\begin{array}{l}
k \\
3
\end{array}\right)}}{\ell\left(\begin{array}{l}
k \\
2
\end{array}\right)} n^{k}(1-\beta) \leq\left|\mathscr{K}_{k}(\mathscr{H})\right| \leq \frac{\alpha^{\left(\begin{array}{c}
k \\
3
\end{array}\right)}}{\ell\left(\begin{array}{l}
k \\
2
\end{array}\right)} n^{k}(1+\beta) .
$$

We also note the following more general theorem, a corollary of which we use in Section 10.3. For arbitrary constants $k, \alpha, \delta, \ell, r, \epsilon$ and $n$ and 3-uniform hypergraph $\mathscr{F}_{0}$ $\subseteq[k]^{3}$, suppose $\mathscr{H}$ and $G=\bigcup_{1 \leq i<j \leq k} G^{i j}$ satisfy Setup 1 . We define the following special set $\mathscr{Y}_{\mathscr{F}_{0}}(\mathscr{H})$ of copies of $\mathscr{F}_{0}$ in $\mathscr{H}$ as

$$
\mathscr{S}_{\mathscr{g}_{0}}(\mathscr{H})=\left\{\mathscr{F} \in\left(\begin{array}{l}
\mathscr{H} \\
\mathscr{F}_{0}
\end{array}\right):[V(\mathscr{F})]^{2} \subseteq G\right\} .
$$

Note that by condition (2) of Setup 1 , for any $\mathscr{F} \in\left(\begin{array}{l}\mathscr{H} \\ \mathscr{F}_{0}\end{array}\right)$, for any $u, v \in V(\mathscr{F}), u \neq v$, the pair $\{u, v\} \in G$ if there exists $w \in V(\mathscr{F})$ with $\{u, v, w\} \in \mathscr{F}$. However, the condition in (17) guarantees that $\{u, v\} \in G$ for all $u, v \in V(\mathscr{F}), u \neq v$.

Then the following may be proved using Theorem 10.8 (see [13]).

Theorem 10.9. For all integers $k \geq 4$ and 3-uniform hypergraphs $\mathscr{f}_{0} \subseteq[k]^{3}$, for all $\alpha$, $\beta>0$, there exists a constant $\delta>0$ so that, for all integers $\ell \geq \frac{1}{\delta}$, there exists an integer $r$ and $\epsilon>0$ so that for $n$ sufficiently large, whenever k-partite 3-cylinder $\mathcal{H}$ and $G$ $=\bigcup_{1 \leq i<j \leq k} G^{i j}$ satisfy the conditions of Setup 1 with constants $k, \alpha, \delta, \ell, r, \epsilon$, and $n$ and 3-uniform hypergraph $\mathscr{F}_{0}$, then

$$
\frac{\alpha^{\left|\mathscr{F}_{0}\right|}}{\ell^{\left(\begin{array}{c}
k \\
2
\end{array}\right)}} n^{k}(1-\beta) \leq\left|\mathscr{S}_{\mathscr{F}_{0}}(\mathscr{H})\right| \leq \frac{\alpha^{\left|\mathscr{F}_{0}\right|}}{\ell^{\left(\begin{array}{c}
k \\
2
\end{array}\right)}} n^{k}(1+\beta) .
$$

We remark that in Theorem 10.9, if $\mathscr{F}_{0}=K_{k}^{(3)}$, then $\left|\mathscr{I}_{\mathscr{F}_{0}}\left(\mathscr{H}_{\mathcal{H}}\right)\right|=\left|\mathscr{K}_{k}(\mathscr{H})\right|$; thus, Theorem 10.8 is an obvious corollary to Theorem 10.9. However, perhaps surprisingly, using a rather straightforward argument, one may derive Theorem 10.9 from Theorem 10.8. In Section 11 we make similar arguments establishing the relationship between the upcoming Theorem 10.12 and Lemma 10.13 of this paper.

The following corollary to Theorem 10.9 is needed for our proof of Theorem 8.2 given in Section 10.3. The following corollary basically states that if $\mathscr{H}^{\prime} \subseteq \mathscr{H}$ is large enough, then the formula in Theorem 10.9 accurately counts the number of cliques $K_{k}^{(3)}$ in $\mathscr{H}^{\prime}$ as well.

Corollary 10.10. For all integers $k \geq 4$ and 3-uniform hypergraphs $\mathscr{F}_{0} \subseteq[k]^{3}$, for all $\alpha, \beta>0$, there exists $\delta=\delta\left(\mathscr{F}_{0}, \alpha, \beta\right)>0$ so that, for all integers $\ell \geq \frac{1}{\delta}$, there exist $r=$ $r\left(\mathscr{F}_{0}, \alpha, \beta, \ell\right), \epsilon=\epsilon\left(\mathscr{F}_{0}, \alpha, \beta, \ell\right)>0$, and integer $n_{0}=n_{0}\left(\mathscr{F}_{0}, \alpha, \beta, \ell\right)$ so that, for all $n \geq$ $n_{0}$, whenever k-partite 3 -cylinder $\mathscr{H}$ and $G=\bigcup_{1 \leq i<j \leq k} G^{i j}$ satisfy the conditions of Setup 1 with constants $k, \alpha, \delta, \ell, r, \epsilon$, and $n$ and 3-uniform hypergraph $\mathscr{F}_{0}$, then, for any subsystem $\mathscr{H}^{\prime} \subseteq \mathscr{H},\left|\mathscr{H}^{\prime}\right|>(1-\mu)|\mathscr{H}|$, where $\mu=\frac{\alpha\left(\begin{array}{l}k \\ 3\end{array}\right) \beta}{24 k^{3}}$, $\mathscr{H}^{\prime}$ satisfies 


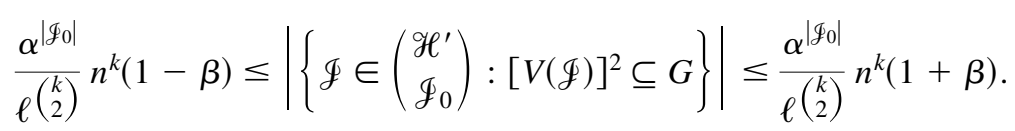

The proof of Corollary 10.10 is easy and we give it in Section 10.4.

We now state an important definition which extends Definition 10.4.

Definition 10.11. As in Setup 1, for a triple $h \in \mathcal{H}$, set

$$
\mathscr{E}_{\mathscr{F}_{0}}(h)=\left\{\mathscr{F} \in \mathscr{Y}_{\mathscr{F}_{0}}(\mathscr{H}): h \in \mathscr{F}\right\} .
$$

In other words, $\mathscr{E}_{\mathscr{F}_{0}}(h)$ is the set of all copies $\mathscr{\mathscr { F }} \in \mathscr{Y}_{\mathscr{F}_{0}}(\mathscr{H})$ which contain $h$ as a triple.

The main result of this section and the remainder of this paper, the Extension Theorem, provides a formula for $\left|\mathscr{E}_{\mathscr{F}_{0}}(h)\right|$ in the setting of Theorem 10.8 .

Theorem 10.12 (Extension Theorem). Let $k \geq 4$ be an integer and let $\mathscr{F}_{0} \subseteq[k]^{3}$, where we assume, without loss of generality, that $\{1,2,3\} \in \mathscr{g}_{0}$. For all reals $\alpha, \mu, \sigma>0$, there exists $\delta=\delta\left(\mathscr{F}_{0}, \alpha, \mu, \sigma\right)>0$ so that, for all integers $\ell>\frac{1}{\delta}$, there exist $r=r\left(\mathscr{F}_{0}, \alpha, \mu\right.$, $\sigma, \ell), \epsilon=\epsilon\left(\mathscr{F}_{0}, \alpha, \mu, \sigma, \ell\right)$, and $n_{0}=n_{0}\left(\mathscr{F}_{0}, \alpha, \mu, \sigma, \ell\right)$ so that whenever $n \geq n_{0}$ and $\mathscr{H}$ and $G=\bigcup_{1 \leq i<j \leq k} G^{i j}$ satisfy the hypothesis of Setup 1 with constants $k, \alpha, \delta, \ell, r, \epsilon$, and $n$ and 3-uniform hypergraph $\mathscr{F}_{0}$, then all but $\mu|\mathcal{H}(\{1,2,3\})|$ triples $h \in \mathscr{H}(\{1,2,3\})$ satisfy

$$
\frac{\alpha^{\left|\mathscr{F}_{0}\right|-1}}{\ell^{\left(\begin{array}{c}
k \\
2
\end{array}\right)-3}} n^{k-3}(1-\sigma) \leq\left|\mathscr{E}_{\mathscr{g}_{0}}(h)\right| \leq \frac{\alpha^{\left|\mathscr{F}_{0}\right|-1}}{\ell^{\left(\begin{array}{c}
k \\
2
\end{array}\right)-3}} n^{k-3}(1+\sigma) .
$$

We now discuss a partial schedule for how we prove Theorem 10.12. To that end, we consider the following Lemma 10.13 which is a special case of Theorem 10.12 when $\mathscr{J}_{0}=$ $K_{k}^{(3)}$ and where we are concerned only with the lower bound for $\left|\mathscr{E}_{K_{k}}^{(3)}(h)\right|$.

Lemma 10.13 (Extension Lemma). Let $k \geq 4$ be an integer. For all reals $\alpha, \mu, \sigma>0$, there exists $\delta>0$ so that, for all integers $\ell>\frac{1}{\delta}$, there exists an integer $r$ and $\epsilon>0$ so that whenever $n$ is sufficiently large and $\mathscr{H}$ and $G=\bigcup_{1 \leq i<j \leq k} G^{i j}$ satisfy the hypothesis of Setup 1 with constants $k, \alpha, \delta, \ell, r, \epsilon$, and $n$ and 3 -uniform hypergraph $\mathscr{E}_{0}=K_{k}^{(3)}$, then all but $\mu|\mathscr{H}(\{1,2,3\})|$ triples $h \in \mathscr{H}(\{1,2,3\})$ satisfy

$$
\left|\mathscr{E}_{K_{k}^{(3)}}(h)\right| \geq \frac{\alpha^{\left(\begin{array}{l}
k \\
3
\end{array}\right)-1}}{\ell^{\left(\begin{array}{l}
k \\
2
\end{array}\right)-3}} n^{k-3}(1-\sigma) .
$$

The schedule is then given as follows.

Remark 10.14 (Theorems 10.12 and 10.13 are equivalent). One readily observes that Theorem 10.12 implies Lemma 10.13. On the other hand, perhaps surprisingly, it is also true that Lemma 10.13 implies Theorem 10.12. This implication is the key to our proof of Theorem 10.12. The schedule of our proof is as follows. 
1. In Section 11, we show that Lemma 10.13 implies Theorem 10.12.

2. Our proof of Lemma 10.13 involves a rather complex argument. We give this argument, in stages, in Sections 12 and 13. We postpone discussing how this argument is divided across Sections 12 and 13 until we are in context.

We close this subsection with following technical comment.

Remark 10.15. We prove Lemma 10.13 for the case when $k \geq 5$ rather than in its fullest generality when $k \geq 4$. We comment that the proof of Lemma 10.13 when $k=4$ is in fact easier than when $k \geq 5$, but its proof would require some of its own notation and space to formalize. Because the details and strategy of the proof for $k=4$ are subsumed and indeed well surpassed by the proof we present for the case $k \geq 5$, we omit them here. The reader is encouraged to see the paper [6] for the essential ingredients of the proof of Lemma 10.13 in the case that $k=4$.

We also mention that not only can our proof of Lemma 10.13 for $k \geq 5$ be altered, indeed simplified, to give the proof for $k=4$, it also true that Lemma 10.13 for $k \geq 5$ implies Lemma 10.13 for $k=4$. A proof of this claim is along straightforward lines. However, for the sake of a fluid exposition, we omit these details as well.

\subsection{Proof of Lemma 8.2}

In this subsection, we give the proof of our required application, Lemma 8.2, using Corollary 10.10 and Theorem 10.12.

Proof of Lemma 8.2. Let $\mathscr{F}_{0}, \lambda$ and $\alpha$ be given. We define $\zeta=\min$ $\left\{\delta_{10.10}\left(\mathscr{F}_{0}, \alpha, \lambda\right), \delta_{10.12}\left(\mathscr{E}_{0}, \alpha, \alpha\left(\begin{array}{c}k \\ 3\end{array}\right) \lambda / 24 k^{3}, \lambda\right)\right\}$. Given $\ell>1 / \zeta$, let

$$
\begin{gathered}
n_{1}=\max \left\{n_{10.10}\left(\mathscr{F}_{0}, \alpha, \lambda, \ell\right), n_{10.12}\left(\mathscr{E}_{0}, \alpha, \alpha\left(\begin{array}{l}
k \\
3
\end{array}\right) \lambda / 24 k^{3}, \lambda, \ell\right)\right\}, \\
r=\max \left\{r_{10.10}\left(\mathscr{E}_{0}, \alpha, \lambda, \ell\right), r_{10.12}\left(\mathscr{E}_{0}, \alpha, \alpha\left(\begin{array}{c}
k \\
3
\end{array}\right) \lambda / 24 k^{3}, \lambda, \ell\right)\right\},
\end{gathered}
$$

and

$$
\epsilon=\min \left\{\epsilon_{10.10}\left(\mathscr{F}_{0}, \alpha, \lambda, \ell\right), \epsilon_{10.12}\left(\mathscr{F}_{0}, \alpha, \alpha\left(\begin{array}{l}
k \\
3
\end{array}\right) \lambda / 24 k^{3}, \lambda, \ell\right)\right\}
$$

Let $\mathscr{H}$ and $G$ be as in the statement of the lemma, and let $\mathscr{H}_{0}$ be the subhypergraph of $\mathscr{H}$ defined by

$$
\mathscr{H}_{0}=\bigcup_{\{i, j, s\} \in \mathscr{F}_{0}} \mathscr{H}\left(G^{i j}, G^{i s}, G^{j s}\right) .
$$

Then, by the hypotheses of the lemma, $\mathscr{H}_{0}$ and $G$ satisfy the hypotheses of Setup 1 with constants $k, \alpha, \zeta, \ell, r, \epsilon$, and $n$ (see the remark before Definition 10.6). Therefore by Theorem 10.12, for each $\{i, j, k\} \in \mathscr{F}_{0}$, all but a $\mu=\alpha\left(\begin{array}{c}k \\ 3\end{array}\right) \lambda / 24 k^{3}$ proportion of the triples in $\mathscr{H}_{0}(\{i, j, k\})$ satisfy $\left|\mathscr{E}_{g_{0}}(h)\right|<(1+\lambda) \alpha^{b-1} n^{k-3} / \ell\left(\begin{array}{l}k \\ 2\end{array}\right)-3$. For each $\{i, j$, 
$k\} \in \mathscr{F}_{0}$, we remove those triples from $\mathscr{H}_{0}(\{i, j, k\})$ which do not satisfy this. We call the resulting subhypergraph $\mathscr{H}^{\prime}$, then $\left|\mathscr{H}^{\prime}\right| \geq(1-\mu)\left|\mathscr{H}_{0}\right|$, and Property (a) holds for $\mathscr{H}^{\prime}$.

Now by Corollary 10.10 , since $\left|\mathscr{H}^{\prime}\right| \geq(1-\mu)\left|\mathscr{H}_{0}\right|$ we have that $\left|\mathscr{I}_{\mathscr{F}_{0}}\left(\mathscr{H}^{\prime}\right)\right|>(1$ $-\lambda) \alpha^{b} n^{k} / \ell\left(\begin{array}{l}k \\ 2\end{array}\right)$, proving Property (b).

\subsection{Proof of Corollary 10.10}

In this subsection, we give the proof of Corollary 10.10 . We begin by defining the promised constants.

Definitions of the Constants. Let integer $k \geq 4$, 3-uniform hypergraph $\mathscr{F}_{0} \subseteq[k]^{3}$ and positives $\alpha, \beta, \mu$ be given where

$$
\mu<\frac{\alpha^{\left(\begin{array}{l}
k \\
3
\end{array}\right)} \beta}{12 k^{3}} .
$$

Define auxiliary constant $\beta^{\prime}$ so that

$$
\beta^{\prime}<\frac{\beta}{3}
$$

Let

$$
\delta=\delta_{10.9}\left(k, \mathscr{F}_{0}, \alpha, \beta^{\prime}\right)
$$

be that constant guaranteed by Theorem 10.9.

Let $\ell>\frac{1}{\delta}$ be a given integer. Let

$$
\begin{aligned}
r & =r_{10.9}\left(k, \mathscr{I}_{0}, \alpha, \beta^{\prime}, \delta, \ell\right), \\
\epsilon^{\prime} & =\epsilon_{10.9}\left(k, \mathscr{I}_{0}, \alpha, \beta^{\prime}, \delta, \ell\right)
\end{aligned}
$$

be those constants guaranteed by Theorem 10.9 . With the constants $k, \ell$, and $\theta=1$, let

$$
\epsilon^{\prime \prime}=\epsilon_{10.5}(k, \ell, \theta)
$$

be that constant guaranteed by Fact 10.5 . Let $\epsilon^{\prime \prime \prime}>0$ satisfy

$$
\frac{\epsilon^{\prime \prime \prime} k^{4} \ell\left(\begin{array}{l}
k \\
2
\end{array}\right)}{\alpha\left(\begin{array}{l}
k \\
3
\end{array}\right)}<\frac{\beta}{3} .
$$

Let

$$
\epsilon^{\prime \prime \prime \prime}=\epsilon_{10.3}(3, \ell, 1)
$$

be that constant guaranteed by Fact 10.3. Set 


$$
\epsilon=\min \left\{\epsilon^{\prime}, \epsilon^{\prime \prime}, \epsilon^{\prime \prime \prime}, \epsilon^{\prime \prime \prime \prime}\right\}
$$

This concludes our definitions of the constants.

Proof of Corollary 10.10. Suppose $k$-partite 3-cylinder $\mathscr{H}$ and $G=\bigcup_{1 \leq i<j \leq k} G^{i j}$ satisfy Setup 1 with constants $k, \alpha, \delta, \ell, r, \epsilon$, and $n$, where $n$ is sufficiently large, and 3-uniform hypergraph $\mathscr{J}_{0} \subseteq[k]^{3}$. We show that, for any $\mathscr{H}^{\prime} \subseteq \mathscr{H},\left|\mathscr{H}^{\prime}\right|>(1-\mu)|\mathscr{H}|$,

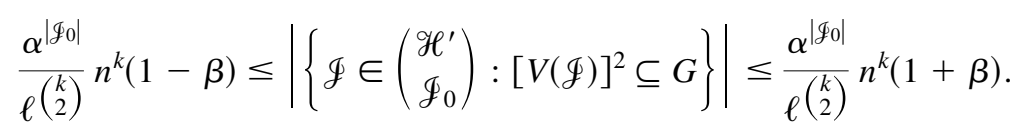

Let $\mathscr{H}^{\prime} \subseteq \mathscr{H},\left|\mathscr{H}^{\prime}\right|>(1-\mu)|\mathscr{H}|$, be given. Define $\mathscr{H}_{1} \subseteq \mathscr{H}^{\prime} \mathscr{H}^{\prime}$ to be the set of all $h=$ $\{x, y, z\} \in \mathscr{H} \backslash \mathscr{H}^{\prime}$ such that the triangle $\{x, y, z\}$ violates the upper bound in (15) with constant $\theta=1$. By Fact 10.5,

$$
\left|\mathscr{H}_{1}\right|<6 \epsilon k\left(\begin{array}{l}
k \\
3
\end{array}\right) n^{3}<\epsilon k^{4} n^{3}
$$

Let $\mathscr{H}_{2}=\left(\mathscr{H}\left(\mathscr{H}^{\prime}\right) \backslash \mathscr{H}_{1}\right.$, and note that all triples $h \in \mathscr{H}_{2}$ satisfy

$$
\left|\mathscr{E}_{K_{k}^{(2)}}^{G}(h)\right|<2 \frac{n^{k-3}}{\ell^{\left(\begin{array}{c}
k \\
2
\end{array}\right)-3}} .
$$

Define sets

$$
\begin{aligned}
& \mathscr{S}=\left\{\mathscr{F} \in \mathscr{Y}_{\mathscr{F}_{0}}(\mathscr{H}): \mathscr{F} \cap\left(\mathscr{H} \backslash \mathscr{H}^{\prime}\right) \neq \varnothing\right\}, \\
& \mathscr{S}_{1}=\left\{\mathscr{F} \in \mathscr{S}: \mathscr{\mathscr { F }} \cap \mathscr{H}_{1} \neq \varnothing\right\}, \\
& \mathscr{S}_{2}=\left\{\mathscr{F} \in \mathscr{S}: \mathscr{\mathscr { F }} \cap \mathscr{H}_{2} \neq \varnothing\right\} \text {, }
\end{aligned}
$$

so that

$$
\mathscr{S}=\mathscr{S}_{1} \cup \mathscr{S}_{2}
$$

Since any $h \in \mathscr{Y}_{1}$ satisfies $\left|\mathscr{E}_{K_{k}^{(2)}}^{G}(h)\right| \leq n^{k-3}$, we see from (21) that

$$
\left|\mathscr{S}_{1}\right|<\epsilon k^{4} n^{k}
$$

Using (22), we see

$$
\left|\mathscr{S}_{2}\right|<2\left|\mathscr{H} \mathscr{H}^{\prime}\right| \frac{n^{k-3}}{\ell\left(\begin{array}{l}
k \\
2
\end{array}\right)-3}
$$

and since $\left|\mathscr{H} \mathscr{H}^{\prime}\right|<\mu|\mathscr{H}|$, where $|\mathscr{H}|=\sum_{B \in[k]^{3}}|\mathscr{H}(B)|<2 k^{3} \frac{n^{3}}{\ell^{3}}$ [cf. (20)], we have 


$$
\left|\mathscr{I}_{2}\right|<4 \mu k^{3} \frac{n^{k}}{\ell^{\left(\begin{array}{l}
k \\
2
\end{array}\right)}}
$$

We now bound $\left|\left\{\mathscr{F} \in\left(\begin{array}{c}\mathscr{F}^{\prime} \\ \mathscr{F}_{0}\end{array}\right):[V(\mathscr{F})]^{2} \subseteq G\right\}\right|$. Using Theorem 10.9,

$$
\frac{\alpha^{\left|\mathscr{F}_{0}\right|}}{\ell^{\left(\begin{array}{c}
k \\
2
\end{array}\right)}} n^{k}\left(1-\beta^{\prime}\right) \leq\left|\mathscr{S}_{\mathscr{F}_{0}}(\mathscr{H})\right| \leq \frac{\alpha^{\left|\mathscr{F}_{0}\right|}}{\ell^{\left(\begin{array}{c}
k \\
2
\end{array}\right)}} n^{k}\left(1+\beta^{\prime}\right),
$$

from which it trivially follows that

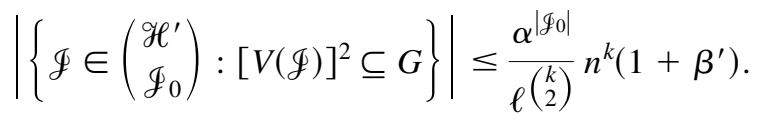

However, we easily see

$$
\left|\left\{\mathscr{F} \in\left(\begin{array}{c}
\mathscr{H}^{\prime} \\
\mathscr{F}_{0}
\end{array}\right):[V(\mathscr{F})]^{2} \subseteq G\right\}\right| \geq\left|\mathscr{S}_{\mathscr{F}_{0}}(\mathscr{H})\right|-|\mathscr{I}| \geq\left|\mathscr{S}_{\mathscr{F}_{0}}(\mathscr{H})\right|-\left|\mathscr{S}_{1}\right|-\left|\mathscr{S}_{2}\right| .
$$

We obtain from (23), (24) and (25) that

$$
\left|\left\{\mathscr{E} \in\left(\begin{array}{c}
\mathscr{H}^{\prime} \\
\mathscr{F}_{0}
\end{array}\right):[V(\mathscr{F})]^{2} \subseteq G\right\}\right| \geq \frac{\alpha^{\left|\mathscr{F}_{0}\right|}}{\ell\left(\begin{array}{l}
k \\
2
\end{array}\right)} n^{k}\left(1-\beta^{\prime}-\frac{\epsilon k^{4} \ell\left(\begin{array}{c}
k \\
2
\end{array}\right)+4 \mu k^{3}}{\alpha\left(\begin{array}{l}
k \\
3
\end{array}\right)}\right) .
$$

However, by (18), (19) and (20), we see

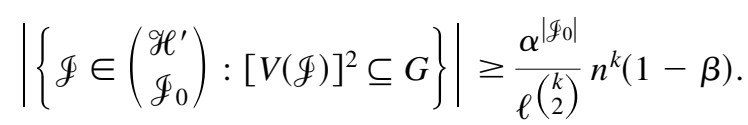

Coupled with (26), the above inequality completes the proof of Corollary 10.10 .

\section{EXTENSION LEMMA IMPLIES EXTENSION THEOREM}

In this section, we show that the Extension Lemma, Lemma 10.13, implies the Extension Theorem, Theorem 10.12. This implication will be shown in steps. We first give a general discussion of these steps before going into details.

The following setup is a slight variation of Setup 1. As a result, we refer it as Setup 1'. Let an integer $k \geq 4$ be given. Unlike Setup 1, we only define Setup $1^{\prime}$ in the case that $\mathscr{g}_{0}=K_{k}^{(3)}$, thus, we from this point onward suppress the mention of the 3 -uniform hypergraph $\mathscr{F}_{0}$ in regard to Setup 1'. For a set of positive reals $\left\{\alpha_{B}: B \in[k]^{3}\right\}$ and positive real $\delta$, for given positive integers $r$ and $\ell$ and positive real $\epsilon$, for a given positive integer $n$, consider the following environment: 


\section{Setup 1'.}

1. $\mathscr{H}$ is a $k$-partite 3 -cylinder with $k$-partition $\left(V_{1}, \ldots, V_{k}\right),\left|V_{1}\right|=\cdots=\left|V_{k}\right|=n$.

2. $G=\bigcup_{1 \leq i<j \leq k} G^{i j}$ is an underlying $(\ell, \epsilon, k)$-cylinder.

3. For all $B \in[k]^{3}, \mathscr{H}(B)$ is $\left(\alpha_{B}, \delta, r\right)$-regular with respect to $G(B)$.

Note that an important difference between Setup 1 and Setup $1^{\prime}$ is that, in the latter, densities over distinct triads are allowed to differ.

The following lemma serves as a bridge between the Extension Lemma and the Extension Theorem. For this reason, we refer to the following lemma as the "Intermediate Lemma."

Lemma 11.1 (Intermediate Lemma). For $k \geq 4$, for all sets $\left\{\alpha_{B}: B \in[k]^{3}\right\}$ of positive reals, for all $\mu, \sigma>0$, there exists $\delta>0$ so that for all integers $\ell>\frac{1}{\delta}$, there exists $r$ and $\epsilon$ so that whenever $n$ is sufficiently large and $\mathscr{H}$ and $G=\bigcup_{1 \leq i<j \leq k} G^{i j}$ satisfy the hypothesis of Setup $1^{\prime}$ with parameters $k,\left\{\alpha_{B}: B \in[k]^{3}\right\}, \delta>0, \ell, r, \epsilon$, and $n$, then all but $\mu|\mathscr{H}(\{1,2,3\})|$ triples $h \in \mathscr{H}(\{1,2,3\})$ satisfy

$$
\frac{\Pi_{B \in[k]]^{\}}\{1,2,3\}} \alpha_{B}}{\ell^{\left(\begin{array}{c}
k \\
2
\end{array}\right)-3}} n^{k-3}(1-\sigma) \leq\left|\mathscr{E}_{K_{k}^{(3)}}(h)\right| \leq \frac{\Pi_{B \in[k]^{3}\{1,2,3\}} \alpha_{B}}{\left.\ell^{(k} \begin{array}{l}
2 \\
2
\end{array}\right)-3} n^{k-3}(1+\sigma) .
$$

Observe that Lemma 11.1 extends Theorem 10.12 from Setup 1 to Setup 1' in the special case $\mathscr{F}_{0}=K_{k}^{(3)}$. It will be easy to show, however, that Lemma 11.1 quickly implies Theorem 10.12 in its entirety. We give a proof that Lemma 11.1 implies Theorem 10.12 in Section 11.3. It remains, therefore, to discuss how the Extension Lemma, Lemma 10.13, implies the Intermediate Lemma, Lemma 11.1.

Lemma 11.1 is a trivial combination of the following two assertions. The first assertion states the lower bound of Lemma 11.1.

Proposition 11.2 (Lower bound of Lemma 11.1). For $k \geq 4$, for all sets $\left\{\alpha_{B}: B \in[k]^{3}\right\}$ of positive reals, for all $\mu, \sigma>0$, there exists $\delta>0$ so that for all integers $\ell>\frac{1}{\delta}$, there exists $r$ and $\epsilon$ so that whenever $n$ is sufficiently large and $\mathcal{H}$ and $G=\bigcup_{1 \leq i<j \leq k} G^{i j}$ satisfy the hypothesis of Setup $1^{\prime}$ with parameters $k,\left\{\alpha_{B}: B \in[k]^{3}\right\}, \delta>0, \ell, r, \epsilon$, and $n$, then all but $\mu|\mathscr{H}(\{1,2,3\})|$ triples $h \in \mathscr{H}(\{1,2,3\})$ satisfy

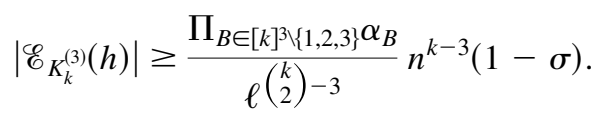

The second assertion states the upper bound of Lemma 11.1.

Proposition 11.3 (Upper bound of Lemma 11.1). For $k \geq 4$, for all sets $\left\{\alpha_{B}: B \in[k]^{3}\right\}$ of positive reals, for all $\mu, \sigma>0$, there exists $\delta>0$ so that for all integers $\ell>\frac{1}{\delta}$, there exists $r$ and $\epsilon$ so that whenever $n$ is sufficiently large and $\mathscr{H}$ and $G=\bigcup_{1 \leq i<j \leq k} G^{i j}$ satisfy the hypothesis of Setup $1^{\prime}$ with parameters $k,\left\{\alpha_{B}: B \in[k]^{3}\right\}, \delta>0, \ell, r, \epsilon$, and $n$, then all but $\mu|\mathscr{H}(\{1,2,3\})|$ triples $h \in \mathscr{H}(\{1,2,3\})$ satisfy 


$$
\left|\mathscr{E}_{K_{k}^{(3)}}(h)\right| \leq \frac{\Pi_{B \in[k]^{3}\{\{1,2,3\}} \alpha_{B}}{e^{\left(\begin{array}{l}
k \\
2
\end{array}\right)-3}} n^{k-3}(1+\sigma) .
$$

To prove Lemma 11.1, it suffices to prove each of Propositions 11.2 and 11.3. In the following remark, we state the strategy and schedule of Section 11.

Remark 11.4. The thesis of Section 11 is as follows: The Extension Lemma, Lemma 10.13, implies the Intermediate Lemma, Lemma 11.1, which in turn implies the Extension Theorem, Theorem 10.12. Symbolically,

$$
\text { Extension Lemma } \Rightarrow \text { Intermediate Lemma } \Rightarrow \text { Extension Theorem. }
$$

1. In Section 11.1, we show the Extension Lemma implies Proposition 11.2.

2. In Section 11.2, we show Proposition 11.2 implies Proposition 11.3.

3. Clearly, Proposition 11.2 and Proposition 11.3 combine to assert the Intermediate Lemma.

4. In Section 11.3, we show the Intermediate Lemma quickly implies the Extension Theorem.

\subsection{The Extension Lemma $\Rightarrow$ Proposition 11.2}

We show that using Lemma 3.5, Proposition 11.2 is just a straightforward corollary of Lemma 10.13. In our proof, we employ Lemma 3.5. To that effect, we recall Lemma 3.5 in language slightly more suitable for our need here.

Lemma 11.5. Let $\alpha, \delta, \epsilon$ be given positive reals with $\delta<\frac{\alpha}{4}$ and let $\ell$ and $r$ be given positive integers. Let $\alpha^{\prime}$ be a given positive real satisfying $\alpha^{\prime} \leq \alpha$, and let 3-partite 3 -cylinder $\mathscr{H}$ and underlying graph $G$ satisfy the hypothesis of Set Up 1 with constants $k=$ $3, \alpha, \delta, \ell, r$, and $\epsilon$. Then there exists a family

$$
\tilde{H}=\left\{\mathscr{H}_{1}, \ldots, \mathscr{H}_{p}\right\}
$$

of 3-partite 3-cylinders with the following properties:

(i) $p=\left\lfloor\frac{\alpha}{\alpha^{\prime}}\right\rfloor$.

(ii) $\mathscr{H}_{i} \cap \mathscr{H}_{j}=\varnothing$ for all $\{i, j\} \in[p]^{2}$, and $\bigcup_{i \in[p]} \mathscr{H}_{i} \subseteq \mathscr{H}_{\text {. }}$

(iii) For each $i \in[p], \mathscr{H}_{i}$ is $\left(\alpha^{\prime}, 2 \delta, r\right)$-regular with respect to the underlying $(\ell$, $\epsilon, 3)$-cylinder $G$.

Proof of Proposition 11.2. Let $k \geq 4$ be a given positive integer and let $\left\{\alpha_{B}: B \in\right.$ $\left.[k]^{3}\right\}$ be a given set of positive reals. Let $\mu, \sigma>0$ be given. Define auxiliary positive constants $\alpha, \sigma^{\prime}$ to satisfy

$$
\left(1-\sigma^{\prime}\right) \Pi_{B \in[k]\}\{1,2,3\}}\left(1-\frac{\alpha}{\alpha_{B}}\right)>1-\sigma,
$$




$$
\alpha<\frac{\mu}{8} \text {. }
$$

Define auxiliary positive constant $\mu^{\prime}$ to satisfy

$$
\frac{2 \mu^{\prime}}{\alpha\left(\begin{array}{l}
k \\
3
\end{array}\right)}<\frac{\mu}{4}
$$

Let

$$
\delta_{10.13}=\delta_{10.13}\left(k, \alpha, \mu^{\prime}, \sigma^{\prime}\right)
$$

be that constant guaranteed by Lemma 10.13 . Set

$$
\delta=\frac{\delta_{10.13}}{2}
$$

Let $\ell>\frac{1}{\delta}$ be a given positive integer. Set

$$
\begin{aligned}
r & =r_{10.13}=r_{10.13}\left(k, \alpha, \mu^{\prime}, \sigma^{\prime}, \delta, \ell\right), \\
\epsilon_{10.13} & =\epsilon_{10.13}\left(k, \alpha, \mu^{\prime}, \sigma^{\prime}, \delta, \ell\right)
\end{aligned}
$$

to be those constants guaranteed by Lemma 10.13 . For the constant $\theta=1 / 4$, set

$$
\epsilon_{10.3}=\epsilon_{10.3}(3, \ell, 1 / 4)
$$

to be that constant guaranteed by Fact 10.3. Set

$$
\epsilon=\min \left\{\epsilon_{10.13}, \epsilon_{10.3}\right\}
$$

For $n$ sufficiently large, suppose $\mathscr{H}$ and $G=\bigcup_{1 \leq i<j \leq k} G^{i j}$ satisfy the hypothesis of Setup 1' with the parameters $k,\left\{\alpha_{B}: B \in[k]^{3}\right\}, \delta>0, \ell, r, \epsilon$, and $n$. We show that all but $\mu|\mathscr{H}(\{1,2,3\})|$ triples $h \in \mathscr{H}(\{1,2,3\})$ satisfy

$$
\left|\mathscr{E}_{K_{k}^{(3)}}(h)\right| \geq \frac{\Pi_{B \in[k]]^{3}\{1,2,3\}} \alpha_{B}}{\ell^{\left(\begin{array}{l}
k \\
2
\end{array}\right)-3}} n^{k-3}(1-\sigma) .
$$

For each $B \in[k]^{3}$, with the constants $\alpha_{B}, \delta$ from (30), $\ell, r$ from (31), $\epsilon$ from (32) and auxiliary constant $\alpha$ from (27) and (28), use Lemma 11.5 to decompose $\mathcal{H}(B)$ into a family

$$
\widetilde{\mathscr{H}(B)}=\left\{\mathscr{H}_{1}(B), \ldots, \mathscr{H}_{p_{B}}(B)\right\}
$$


$p_{B}=\left\lfloor\frac{\alpha_{B}}{\alpha}\right\rfloor$, of pairwise disjoint 3-partite 3-cylinders, each of which is $(\alpha, 2 \delta, r)$-regular with respect to the $(\ell, \epsilon, 3)$-cylinder $G(B)$. Define

$$
\mathscr{I}=\left\{\left(i_{B}\right): B \in[k]^{3}, 1 \leq i_{B} \leq\left\lfloor\frac{\alpha_{B}}{\alpha}\right\rfloor\right\}
$$

to be the set of all vectors of length $\left(\begin{array}{l}k \\ 3\end{array}\right)$ whose $B$-coordinate is an integer $i_{B}$ as given above. For convenience of notation, we denote the $B$-coordinate of a vector $\vec{i} \in \mathscr{I}$ by $\vec{\imath}(B)$. For $1 \leq i_{\{1,2,3\}} \leq p_{\{1,2,3\}}$, define $\mathscr{I}\left(i_{\{1,2,3\}}\right)=\left\{\vec{\imath} \in \mathscr{I}: \vec{l}(\{1,2,3\})=i_{\{1,2,3\}}\right\}$. For each

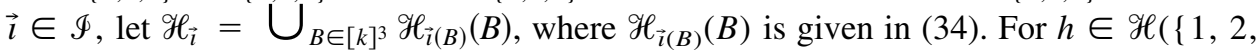
$3\})$, let

$$
\psi(h)=\left\{\begin{array}{ll}
i_{\{1,2,3\}} & \text { if } h \in \mathscr{H}_{i\{1,2,3\}}(\{1,2,3\}) \\
0 & \text { if } h \notin \bigcup_{1 \leq i\{1,2,3\} \leq p\{1,2,3\}}
\end{array} \mathscr{H}_{i\{1,2,3\}}(\{1,2,3\}),\right\}
$$

where $p_{\{1,2,3\}}=\left\lfloor\frac{\alpha_{\{1,2,3\}}}{\alpha}\right\rfloor$.

Fix $\vec{\imath} \in \mathscr{I}$. For $h \in \mathscr{H}_{\vec{l}(\{1,2,3\})}(\{1,2,3\})$, define

$$
\mathscr{E}_{K_{k}^{(3)}}^{\mathscr{H}_{i}}(h)=\left\{Y \in \mathscr{K}_{k}\left(\mathscr{H}_{i}\right): h \in[Y]^{3}\right\} .
$$

Note that $\mathscr{E}_{K_{k}^{(3)}}^{\mathscr{H} \vec{f}_{i}}(h)$ is just the set of copies of $K_{k}^{(3)}$ in $\mathscr{H}_{\vec{i}}$ which contain the triple $h$.

We first show that for the fixed $\vec{\imath} \in \mathscr{I}$, Lemma 10.13 implies that most triples $h \in$ $\mathscr{H}_{\vec{i}(\{1,2,3\})}(\{1,2,3\})$ are such that the set $\mathscr{E}_{K_{k}^{(3)}}^{\mathscr{H}_{\vec{i}}}(h)$ is large enough. Indeed, note that $\mathscr{H}_{\vec{i}}$ satisfies the hypothesis of Setup 1 with constants $k, \alpha, 2 \delta \leq \delta_{10.13}$ [cf. (30)], $\ell, r=$ $r_{10.13}$ [cf. (31)], and $\epsilon=\epsilon_{10.13}$ [cf. (11.1)]. As a result of our constants $k, \alpha, 2 \delta \leq \delta_{10.13}$, $\ell, r=r_{10.13}$, and $\epsilon=\epsilon_{10.13}$ appropriately given, we apply Lemma 10.13 to $\mathscr{H}_{\vec{\imath}}$ to conclude that all but $\mu^{\prime}\left|\mathscr{H}_{\vec{i}(\{1,2,3\})}(\{1,2,3\})\right|$ triples $h \in \mathscr{H}_{\vec{i}(\{1,2,3\})}(\{1,2,3\})$ satisfy

$$
\left|\mathscr{E}_{K_{k}^{(3)}}^{\mathscr{H}_{i}}(h)\right| \geq \frac{\alpha^{\left(\begin{array}{l}
k \\
3
\end{array}\right)-1}}{\ell^{\left(\begin{array}{l}
k \\
2
\end{array}\right)-3}} n^{k-3}\left(1-\sigma^{\prime}\right) .
$$

Our next step is to show that whenever $h \in \mathcal{H}(\{1,2,3\})$ satisfies the inequality in (36) for every $\vec{\imath} \in \mathscr{I}(\psi(h))$, then $h$ also satisfies (33). To that effect, note that for $1 \leq$ $i_{\{1,2,3\}} \leq p_{\{1,2,3\}}$,

$$
\left|\mathscr{I}\left(i_{\{1,2,3\}}\right)\right|=\Pi_{B \in[k]\} \backslash\{1,2,3\}}\left\lfloor\frac{\alpha_{B}}{\alpha}\right\rfloor .
$$

Define

$$
\mathscr{H}_{\text {good }}(\{1,2,3\})=\{h \in \mathscr{H}(\{1,2,3\}): \psi(h) \neq 0 \text { and } h \text { satisfies (36) for all } \vec{\imath} \in \mathscr{I}(\psi(h))\}
$$




$$
\mathscr{H}_{\text {bad }}(\{1,2,3\})=\mathscr{H}(\{1,2,3\}) \backslash \mathscr{H}_{\text {good }}(\{1,2,3\}) .
$$

As an immediate consequence of (36), for each $h \in \mathscr{H}_{\text {good }}(\{1,2,3\})$,

$$
\begin{aligned}
& \left|\mathscr{E}_{K_{k}^{(3)}}(h)\right| \geq \sum_{\vec{i} \in \mathscr{I}(\psi(h))}\left|\mathscr{E}_{K_{k}^{(3)}}^{\mathscr{K}_{\vec{i}}}(h)\right| \\
& \geq \frac{\alpha^{\left(\begin{array}{l}
k \\
3
\end{array}\right)-1}}{\ell^{\left(\begin{array}{l}
k \\
2
\end{array}\right)-3}} n^{k-3}\left(1-\sigma^{\prime}\right)|\mathscr{I}(\psi(h))| \\
& =\frac{\alpha^{\left(\begin{array}{l}
k \\
3
\end{array}\right)-1}}{\ell^{\left(\begin{array}{l}
k \\
2
\end{array}\right)-3}} n^{k-3}\left(1-\sigma^{\prime}\right) \prod_{B \in[k]\} \backslash\{1,2,3\}}\left\lfloor\frac{\alpha_{B}}{\alpha}\right\rfloor \\
& \geq \frac{\Pi_{B \in[k]^{3}\{1,2,3\}} \alpha_{B}}{\left.\ell^{k} \begin{array}{l}
2 \\
2
\end{array}\right)-3} n^{k-3}\left(1-\sigma^{\prime}\right) \Pi_{B \in[k]^{3} \backslash\{1,2,3\}}\left(1-\frac{\alpha}{\alpha_{B}}\right) \text {. }
\end{aligned}
$$

As a result of our choice of $\alpha$ in (27), we further conclude

$$
\left|\mathscr{E}_{K_{k}^{(3)}}(h)\right| \geq \frac{\Pi_{B \in[k]^{3} \backslash\{1,2,3\}} \alpha_{B}}{l_{\left(\begin{array}{l}
k \\
2
\end{array}\right)-3}} n^{k-3}(1-\sigma) .
$$

With each $h \in \mathscr{H}_{\text {good }}(\{1,2,3\})$ satisfying (37), all we need to show to complete the

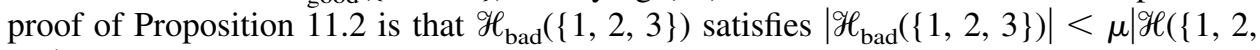
$3\})$.

We begin by estimating the number of triples $h \in \mathscr{H}(\{1,2,3\})$ for which $\psi(h) \neq 0$ and where there exists $\vec{\imath} \in \mathscr{I}(\psi(h))$ for which $h$ and $\vec{\imath}$ fail to satisfy (36). To that end, recall that Lemma 10.13 ensures that for each $1 \leq i_{\{1,2,3\}} \leq\left\lfloor\frac{\alpha_{\{1,2,3\}}}{\alpha}\right\rfloor$, for each $\vec{\imath} \in$ $\Phi\left(i_{\{1,2,3\}}\right)$, all but $\mu^{\prime}\left|\mathscr{H}_{i_{\{1,2,3\}}}(\{1,2,3\})\right|$ triples $h \in \mathscr{H}_{i_{\{1,2,3\}}}(\{1,2,3\})$ satisfy (36). For $1 \leq i_{\{1,2,3\}} \leq p_{\{1,2,3\}}$, define $\mathscr{H}_{\text {bad, } i_{\{1,2,3\}}}(\{1,2,3\})$ to be the set of all $h \in \mathscr{H}_{i_{\{1,2,3\}}}(\{1$, $2,3\})$ such that there exists $\vec{\imath} \in \mathscr{I}\left(i_{\{1,2,3\}}\right)$ for which $h$ and $\vec{\imath}$ fail to satisfy (36). We wish to bound

$$
\left|\bigcup_{1 \leq i\{1,2,3\} \leq p\{1,2,3\}} \mathscr{H}_{\text {bad }, i\{1,2,3\}}(\{1,2,3\})\right| .
$$

We first note that by Lemma 10.13 , for $i_{\{1,2,3\}}$ fixed,

$$
\left|\mathscr{H}_{\text {bad }, i\{1,2,3\}}(\{1,2,3\})\right| \leq \mu^{\prime}\left|\mathscr{H}_{i\{1,2,3\}}(\{1,2,3\})\right|\left|\mathscr{S}\left(i_{\{1,2,3\}}\right)\right| .
$$

Note that since $\mathscr{H}_{i_{\{1,2,3\}}}(\{1,2,3\})$ is $(\alpha, 2 \delta, r)$-regular with respect to $G^{12} \cup G^{13} \cup G^{23}$, then

$$
\left|\mathscr{H}_{i\{1,2,3\}}(\{1,2,3\})\right| \leq(\alpha+2 \delta)\left|\mathscr{K}_{3}\left(G^{12} \cup G^{13} \cup G^{23}\right)\right| \leq 2 \alpha \frac{n^{3}}{\ell^{3}},
$$

where the last inequality follows from Fact 10.3. Thus, we see that 


$$
\begin{aligned}
\left|\mathscr{H}_{\mathrm{bad}, i\{1,2,3\}}(\{1,2,3\})\right| & \leq 2 \mu^{\prime} \alpha \frac{n^{3}}{\ell^{3}}\left|\Phi\left(i_{\{1,2,3\}}\right)\right|, \\
& =2 \mu^{\prime} \alpha \frac{n^{3}}{\ell^{3}} \Pi_{B \in[k]\}\{1,2,3\}}\left\lfloor\frac{\alpha_{B}}{\alpha}\right\rfloor .
\end{aligned}
$$

Thus, with $p_{\{1,2,3\}}=\left\lfloor\frac{\alpha_{\{1,2,3\}}}{\alpha}\right\rfloor$,

$$
\left|\bigcup_{1 \leq i \leq p\{1,2,3\}} \mathscr{H}_{\mathrm{bad}, i\{1,2,3\}}(\{1,2,3\})\right| \leq 2 \mu^{\prime} \alpha \frac{n^{3}}{\ell^{3}} \Pi_{B \in[k]^{3}}\left\lfloor\frac{\alpha_{B}}{\alpha}\right\rfloor .
$$

Note that $\mathscr{H}_{\mathrm{bad}}(\{1,2,3\}) \backslash \bigcup_{1 \leq i \leq p\{1,2,3\}} \mathscr{H}_{\mathrm{bad}, i\{1,2,3\}}(\{1,2,3\})$ is just the set of all triples $h \in \mathscr{H}(\{1,2,3\})$ for which $\psi(h)=0$. However, by Property (i) of Lemma 11.5, we see that

$$
\left|\mathscr{H}_{\text {bad }}(\{1,2,3\}) \backslash \bigcup_{1 \leq i \leq p\{1,2,3\}} \mathscr{H}_{\text {bad }, i\{1,2,3\}}(\{1,2,3\})\right| \leq \alpha|\mathscr{H}(\{1,2,3\})| .
$$

As a result of (38) and (39), we see

$$
\begin{aligned}
\left|\mathscr{H}_{\mathrm{bad}}(\{1,2,3\})\right| \leq & \left|\bigcup_{1 \leq i \leq p\{1,2,3\}} \mathscr{H}_{\mathrm{bad}, i\{1,2,3\}}(\{1,2,3\})\right| \\
& +\left|\mathscr{H}_{\mathrm{bad}}(\{1,2,3\}) \backslash \bigcup_{1 \leq i \leq p\{1,2,3\}} \mathscr{H}_{\mathrm{bad}, i\{1,2,3\}}(\{1,2,3\})\right|, \\
< & \left.2 \alpha \mu^{\prime} \frac{n^{3}}{\ell^{3}} \Pi_{B \in[k]^{3}} \mid \frac{\alpha_{B}}{\alpha}\right]+2 \frac{n^{3}}{\ell^{3}} \alpha \alpha_{\{1,2,3\}}, \\
\leq & \alpha_{\{1,2,3\}} \frac{n^{3}}{\ell^{3}}\left[\frac{2 \mu^{\prime}}{\alpha\left(\begin{array}{c}
k \\
3
\end{array}\right)-1}+2 \alpha\right] .
\end{aligned}
$$

As a result of (28) and (29), we further conclude

$$
\left|\mathscr{H}_{\text {bad }}(\{1,2,3\})\right| \leq \alpha_{\{1,2,3\}} \frac{n^{3}}{\ell^{3}}\left[\frac{\mu}{4}+\frac{\mu}{4}\right]=\frac{\mu}{2} \alpha_{\{1,2,3\}} \frac{n^{3}}{\ell^{3}} .
$$

Since $\mathscr{H}(\{1,2,3\})$ is $\left(\alpha_{\{1,2,3\}}, \delta, r\right)$-regular with respect to $G^{12} \cup G^{13} \cup G^{23}$, we see that

$$
|\mathcal{H}(\{1,2,3\})| \geq\left(\alpha_{\{1,2,3\}}-\delta\right)\left|\mathscr{K}_{3}\left(G^{12} \cup G^{13} \cup G^{23}\right)\right|>\frac{\alpha_{\{1,2,3\}}}{2} \frac{n^{3}}{\ell^{3}},
$$

where the last inequality follows from Fact 10.3. Thus, comparing (40) with (41), we conclude 


$$
\left|\mathscr{H}_{\text {bad }}(\{1,2,3\})\right|<\mu|\mathscr{H}(\{1,2,3\})|,
$$

and the proof of Proposition 11.2 is complete.

\subsection{Proposition 11.2 $\Rightarrow$ Proposition 11.3}

We show Proposition 11.3 quickly follows from Proposition 11.2. We begin by defining the promised constants.

Definitions of the Constants. Let $k \geq 4$ be a given integer, let $\left\{\alpha_{B}: B \in[k]^{3}\right\}$ be a set of positive reals, and let $\mu, \sigma>0$ be given. Define auxiliary positive constants $\mu^{\prime}, \sigma^{\prime}$, and $\theta$ to satisfy

$$
\begin{gathered}
\frac{\theta+\sigma^{\prime}}{\prod_{B \in[k]^{3} \alpha_{B}}}<\sigma, \\
\left(2\left(\begin{array}{l}
k \\
3
\end{array}\right)+1\right) \mu^{\prime}<\frac{\mu}{2} .
\end{gathered}
$$

Let $\mathscr{F}$ be the set of all binary sequences of length $\left(\begin{array}{c}k \\ 3\end{array}\right)$. For each $\vec{\jmath} \in \mathscr{F}$, for each $B \in$ $[k]^{3}$, denote by $\vec{j}(B)$ the $B$-coordinate of $\vec{\jmath}$. For $\vec{j} \in \mathscr{J}$, define $\mathscr{A}(\vec{\jmath})=\left\{\alpha_{B}(\vec{\jmath}): B \in\right.$ $\left.[k]^{3}\right\}$, where, for each $B \in[k]^{3}$,

$$
\alpha_{B}(\vec{\jmath})= \begin{cases}\alpha_{B} & \text { if } \vec{\jmath}(B)=1 \\ 1-\alpha_{B} & \text { if } \vec{\jmath}(B)=0\end{cases}
$$

For $\vec{j} \in \mathscr{g}$, for the parameters $k, \mathscr{A}(\vec{\jmath}), \mu^{\prime}, \sigma^{\prime}$, let

$$
\delta_{11.2}^{(\vec{j})}=\delta_{11.2}^{(\vec{j})}\left(k, \mathscr{A}(\vec{\jmath}), \mu^{\prime}, \sigma^{\prime}\right)
$$

be the constant guaranteed by Proposition 11.2. Set

$$
\delta=\min _{\vec{j} \in \mathscr{q}} \delta_{11.2}^{(\vec{j})}
$$

Let $\ell>\frac{1}{\delta}$ be a given integer. For $\vec{j} \in \mathscr{F}$, for the parameters $k, \mathscr{A}(\vec{\jmath}), \mu^{\prime}, \sigma^{\prime}, \delta$, and $\ell$, let

$$
\begin{aligned}
& r_{11.2}^{(\vec{j})}=r_{11.2}^{(\vec{j})}\left(k, \mathscr{A}(\vec{\jmath}), \mu^{\prime}, \sigma^{\prime}, \delta, \ell\right), \\
& \epsilon_{11.2}^{(\vec{j})}=\epsilon_{11.2}^{(\vec{\jmath})}\left(k, \mathscr{A}(\vec{\jmath}), \mu^{\prime}, \sigma^{\prime}, \delta, \ell\right)
\end{aligned}
$$

be those constants guaranteed by Proposition 11.2. Set 


$$
\begin{gathered}
r=\max _{\vec{j} \in \mathscr{q}} r_{11.2}^{(\vec{j})}, \\
\epsilon_{1}=\min _{\vec{j} \in \mathscr{q}} \epsilon_{11.2}^{(\vec{j})} .
\end{gathered}
$$

For $k, \theta$, and $\ell$ given above, let

$$
\epsilon^{\prime}=\epsilon_{10.5}(k, \theta, \ell)
$$

be that constant guaranteed by Fact 10.5 . Let $\epsilon^{\prime \prime}>0$ satisfy

$$
6 k \epsilon^{\prime \prime} \ell^{3}<\mu^{\prime} \frac{\alpha_{\{1,2,3\}}}{2}
$$

Let

$$
\epsilon^{\prime \prime \prime}=\epsilon_{10.3}\left(3, \ell, \frac{1}{4}\right)
$$

be that constant guaranteed by Fact 10.3. Set

$$
\epsilon=\min \left\{\epsilon_{1}, \epsilon^{\prime}, \epsilon^{\prime \prime}, \epsilon^{\prime \prime \prime}\right\}
$$

This concludes our definitions of the constants.

Proof of Proposition 11.3. Suppose that $\mathscr{H}$ and $G=\bigcup_{1 \leq i<j \leq k} G^{i j}$ satisfy the hypothesis of Setup 1' with parameters $k,\left\{\alpha_{B}: B \in[k]^{3}\right\}, \delta>0, \ell, r, \epsilon$, and $n$ sufficiently large. We show that all but $\mu|\mathscr{H}(\{1,2,3\})|$ triples $h \in \mathscr{H}(\{1,2,3\})$ satisfy

$$
\left|\mathscr{E}_{K_{k}^{(3)}}(h)\right| \leq \frac{\Pi_{B \in[k]]^{3}\{1,2,3\}} \alpha_{B}}{\ell^{\left(\begin{array}{l}
k \\
2
\end{array}\right)-3}} n^{k-3}(1+\sigma) .
$$

To that end, recall we earlier defined $\mathscr{F}$ to be the set of all binary sequences of length $\left(\begin{array}{c}k \\ 3\end{array}\right)$. For each $\vec{j} \in \mathscr{J}$, consider $\mathscr{H}_{\vec{j}}=\bigcup_{B \in[k]^{3}} \mathscr{H}_{\vec{j}}(B)$, where

$$
\mathscr{H}_{\vec{\jmath}}(B)= \begin{cases}\mathscr{H}_{(}(B) & \text { if } \vec{\jmath}(B)=1 \\ \mathscr{K}_{3}(G(B)) \backslash \mathcal{H}(B) & \text { if } \vec{\jmath}(B)=0 .\end{cases}
$$

Note that if $\vec{j}(B)=1$, then $d_{\mathscr{H}_{j}}(G(B))=\alpha_{B}$, and otherwise, $d_{\mathscr{H}_{j}}(G(B))=1-\alpha_{B}$.

For convenience of notation, set $\mathscr{g}_{1}=\{\vec{j} \in \mathscr{g}: \vec{\jmath}(\{1,2,3\})=1\}$. Fix $\vec{j} \in \mathscr{F}_{1}$. For a triple $h \in \mathcal{H}(\{1,2,3\})$, define

$$
\mathscr{E}_{K_{k}^{(3)}}^{\mathscr{H}_{j}}(h)=\left\{Y \in \mathcal{K}_{k}\left(\mathscr{H}_{\vec{j}}\right): h \in[Y]^{3}\right\} .
$$


Define $\mathscr{H}^{\text {(good) }}(\{1,2,3\}) \subseteq \mathscr{H}(\{1,2,3\})$ to be the set of triples $h=\{x, y, z\} \in \mathscr{H}(\{1$, $2,3\})$ which satisfy the conclusion of Fact 10.5 with constant $\theta$ from (42) and such that for every choice $\vec{j} \in \mathscr{F}_{1}, h$ satisfies

$$
\left|\mathscr{E}_{K_{k}^{(3)}}^{\mathscr{K}_{j}}(h)\right| \geq \frac{\Pi_{B \in[k]^{3} \backslash\{1,2,3\}} \alpha_{B}(\vec{\jmath})}{\ell^{\left(\begin{array}{l}
k \\
2
\end{array}\right)-3}} n^{k-3}\left(1-\sigma^{\prime}\right),
$$

where $\alpha_{B}(\vec{\jmath})$ is given in (44). Then, for each $h \in \mathscr{H}^{\text {(good) }}(\{1,2,3\})$, observe

$$
\sum_{j \in \mathscr{F}_{1}}\left|\mathscr{E}_{K_{k}^{(3)}}^{\mathscr{H}_{j}}(h)\right|=\left|\mathscr{E}_{K_{k}^{(3)}}^{G}(h)\right| \leq \frac{n^{k-3}}{\ell\left(\begin{array}{l}
k \\
2
\end{array}\right)-3}(1+\theta),
$$

where the last inequality follows from Fact 10.5 and our choice of $\epsilon$ in (47). Denoting $\overrightarrow{1}=$ $(1, \ldots, 1) \in \mathscr{F}_{1}$, the sequence of all 1's, we see by Proposition 11.2 that for $h \in$ $\mathcal{H}^{\text {(good) }}(\{1,2,3\})$,

$$
\begin{aligned}
& \frac{n^{k-3}}{\ell^{\left(\begin{array}{l}
k \\
2
\end{array}\right)-3}}(1+\theta) \geq\left|\mathscr{E}_{K_{k}^{(3)}}^{\mathscr{H}_{\overrightarrow{1}}}(h)\right|+\sum_{\vec{j} \in \mathscr{F}_{1} \backslash\{\overrightarrow{1}\}}\left|\mathscr{E}_{K_{k}^{(3)}}^{\mathscr{H}_{\vec{j}}}(h)\right| \\
& \geq\left|\mathscr{E}_{K_{k}^{(3)}}^{\mathscr{H}_{1}}(h)\right|+\sum_{\vec{\jmath} \in \mathscr{F}_{1} \backslash\{1\}} \frac{\Pi_{B \in[k]\} \backslash\{1,2,3\}} \alpha_{B}(\vec{\jmath})}{\ell\left(\begin{array}{l}
k \\
2
\end{array}\right)-3} n^{k-3}\left(1-\sigma^{\prime}\right) .
\end{aligned}
$$

As $\mathscr{H}_{\hat{1}}=\mathscr{H}$, note that $\mathscr{E}_{K_{k}^{(3)}}^{\mathscr{H}}(h)=\mathscr{E}_{K_{k}^{(3)}}(h)$. It thus follows from (49) that

$$
\left|\mathscr{E}_{K_{k}^{(3)}}(h)\right| \leq \frac{n^{k-3}}{\ell\left(\begin{array}{l}
k \\
2
\end{array}\right)-3}\left[1+\theta-\left(1-\sigma^{\prime}\right) \sum_{\vec{\jmath} \in \mathscr{F}_{1} \backslash\{\overrightarrow{1}\}} \Pi_{B \in[k]} \backslash\{1,2,3\} \alpha_{B}(\vec{\jmath})\right] .
$$

Note that with $\alpha_{B}(\vec{\jmath})$ defined in (44), $B \in[k]^{3} \backslash\{1,2,3\}$, we have

$$
\sum_{\vec{\jmath} \in \mathscr{F}_{1}} \Pi_{B \in[k]^{3} \backslash\{1,2,3\}} \alpha_{B}(\vec{\jmath})=\Pi_{B \in[k]^{3} \backslash\{1,2,3\}}\left(\alpha_{B}+\left(1-\alpha_{B}\right)\right)=1 .
$$

Thus, we conclude from (50) and (51) that

$$
\left|\mathscr{E}_{K_{k}^{(3)}}(h)\right| \leq \frac{n^{k-3}}{\ell\left(\begin{array}{l}
k \\
2
\end{array}\right)-3}\left[1+\theta-\left(1-\sigma^{\prime}\right)\left(1-\Pi_{B \in[k]^{3} \backslash\{1,2,3\}} \alpha_{B}(\overrightarrow{1})\right)\right] .
$$

However, for each $B \in[k]^{3}, \alpha_{B}(\overrightarrow{1})=\alpha_{B}$; thus

$$
\begin{aligned}
\left|\mathscr{E}_{K_{k}^{(3)}}(h)\right| & \leq \frac{n^{k-3}}{\ell\left(\begin{array}{l}
k \\
2
\end{array}\right)-3}\left[\Pi_{B \in[k]\}^{3}\{1,2,3\}} \alpha_{B}+\theta+\sigma^{\prime}\right] \\
& =\prod_{B \in[k]\}\{1,2,3\}} \alpha_{B} \frac{n^{k-3}}{\ell^{\left(\begin{array}{l}
k \\
2
\end{array}\right)-3}}\left[1+\frac{\theta+\sigma^{\prime}}{\prod_{B \in[k]\}\{1,2,3\}} \alpha_{B}}\right] .
\end{aligned}
$$


With our choice of $\theta$ and $\sigma^{\prime}$ in (42), we see that, for each $h \in \mathscr{H}^{(\text {good) }}(\{1,2,3\})$,

$$
\left|\mathscr{E}_{k}^{(3)}(h)\right| \leq \Pi_{B \in[k]\}\{1,2,3\}} \alpha_{B} \frac{n^{k-3}}{\ell^{\left(\begin{array}{c}
k \\
2
\end{array}\right)-3}}(1+\sigma) .
$$

Thus, to prove Proposition 11.3, we need only show $\left|\mathscr{H}(\{1,2,3\}) \backslash \mathscr{H}^{(\text {good })}(\{1,2,3\})\right|$ $<\mu|\mathscr{H}(\{1,2,3\})|$. However, recall that $\mathscr{H}(\{1,2,3\}) \backslash \mathscr{H}^{\text {(good) }}(\{1,2,3\})$ is just the set of triples $h=\{x, y, z\} \in \mathscr{H}(\{1,2,3\})$ which for some $\vec{\jmath} \in \mathscr{F}_{1}$ fail to satisfy (48) or fail to satisfy Fact 10.5 with the constant $\theta$.

However, for each $\vec{j} \in \mathscr{F}_{1}$, due to our choices of $\delta$ in (45), $r$ in (46) and $\epsilon$ in (47), it follows that we may apply Proposition 11.2 to $\mathscr{H}_{\vec{j}}$ and $G=\bigcup_{1 \leq i<j \leq k} G^{i j}$ to conclude that all but $\mu^{\prime}|\mathscr{H}(\{1,2,3\})|$ triples $h \in \mathscr{H}(\{1,2,3\})$ satisfy (48). Since all but $6 k \epsilon n^{3}<\mu^{\prime}$ $\frac{\alpha_{\{1,2,3\}}}{2} \frac{n^{3}}{l^{3}}<\mu^{\prime}|\mathscr{H}(\{1,2,3\})|$ [cf. (47)] triples $h \in \mathscr{H}(\{1,2,3\})$ satisfy the conclusion of Fact 10.5 with the constant $\theta$, we conclude

$$
\left|\mathscr{H}(\{1,2,3\}) \backslash \mathscr{H}^{\text {(good) }}(\{1,2,3\})\right|<\left(2\left(\begin{array}{l}
k \\
3
\end{array}\right)+1\right) \mu^{\prime}|\mathscr{H}(\{1,2,3\})| \leq \mu|\mathscr{H}(\{1,2,3\})|,
$$

where the last inequality follows from our choice of $\mu^{\prime}$ in (43). Thus, the proof of Proposition 11.3 is complete.

\subsection{Intermediate Lemma $\Rightarrow$ Extension Theorem}

We use Lemma 11.1 to prove Theorem 10.12.

Proof of Theorem 10.12. Let $k \geq 4$ be an integer and let $\mathscr{F}_{0} \subseteq[k]^{3}$ be a 3-uniform hypergraph containing $\{1,2,3\}$. Let $\alpha, \mu$, and $\sigma$ be given constants. Set

$$
\delta=\delta_{11.1}(k,\{\alpha\}, \mu, \sigma)
$$

to be that constant guaranteed by Lemma 11.1. Let $\ell>\frac{1}{\delta}$ be a given integer. Set

$$
\begin{aligned}
& r=r_{11.1}(k,\{\alpha\}, \mu, \sigma, \delta, \ell), \\
& \epsilon=\epsilon_{11.1}(k,\{\alpha\}, \mu, \sigma, \delta, \ell),
\end{aligned}
$$

to be those constants guaranteed by Lemma 11.1. Suppose $\mathscr{H}$ and $G=\bigcup_{1 \leq i<j \leq k} G^{i j}$ satisfy the hypothesis of Setup 1 with parameters $k, \alpha, \delta>0, \ell, r, \epsilon$, and $n$, where $n$ is sufficiently large, and 3-uniform hypergraph $\mathscr{F}_{0}$. We show that all but $\mu|\mathscr{H}(\{1,2,3\})|$ triples $h \in \mathscr{H}(\{1,2,3\})$ satisfy

$$
\frac{\alpha^{\left|\mathscr{F}_{0}\right|-1}}{\ell\left(\begin{array}{l}
k \\
2
\end{array}\right)-3} n^{k-3}(1-\sigma) \leq\left|\mathscr{E}_{\mathscr{g}_{0}}(h)\right| \leq \frac{\alpha^{\left|\mathscr{F}_{0}\right|-1}}{\ell\left(\begin{array}{l}
k \\
2
\end{array}\right)-3} n^{k-3}(1+\sigma) .
$$

To that effect, we construct the following auxiliary 3-cylinder $\mathscr{H}_{\mathscr{g}_{0}}$ $=\bigcup_{B \in[k]^{3}} \mathscr{H}_{\mathscr{g}_{0}}(B)$, where 


$$
\mathscr{H}_{\mathscr{F}_{0}}(B)= \begin{cases}\mathscr{H}(B) & \text { if } B \in \mathscr{F}_{0} \\ \mathscr{K}_{3}(G(B)) & \text { if } B \notin \mathscr{E}_{0} .\end{cases}
$$

Note that $\mathscr{H}_{\mathscr{F}_{0}}(\{1,2,3\})=\mathscr{H}(\{1,2,3\})$ (by the hypothesis of Theorem 10.12). Note that, for every triple $h \in \mathscr{H}(\{1,2,3\})$,

$$
\mathscr{E}_{\mathscr{F}_{0}}(h)=\mathscr{E}_{K_{k}^{(3)}}^{\mathscr{H}_{\mathscr{F}_{0}}}(h)
$$

Note that $\mathscr{H}_{\mathscr{F}_{0}}$ and $G=\bigcup_{1 \leq i<j \leq k} G^{i j}$ satisfy the hypothesis of Lemma 11.1 with the following parameters: $k$, set $\left\{\alpha_{B}: B \in[k]^{3}\right\}$, where

$$
\alpha_{B}= \begin{cases}\alpha & \text { if } B \in \mathscr{I}_{0} \\ 1 & \text { if } B \notin \mathscr{I}_{0}\end{cases}
$$

$\mu, \sigma, \delta \leq \delta_{11.1}\left(k,\left\{\alpha_{B}: B \in[k]^{3}\right\}, \mu, \sigma\right), \ell, r \geq r_{11.1}\left(k,\left\{\alpha_{B}: B \in[k]^{3}\right\}, \mu, \sigma\right.$, $\delta, \ell)$, and $\epsilon \leq \epsilon_{11.1}\left(k,\left\{\alpha_{B}: B \in[k]^{3}\right\}, \mu, \sigma, \delta, \ell\right)$. Thus, by Lemma 11.1, all but $\mu\left|\mathscr{H}_{\mathscr{F}_{0}}(\{1,2,3\})\right|$ triples $h \in \mathscr{H}_{\mathscr{f}}(\{1,2,3\})=\mathscr{H}(\{1,2,3\})$ satisfy

$$
\frac{\Pi_{B \in[k]]^{3}\{1,2,3\}} \alpha_{B}}{\ell^{\left(\begin{array}{l}
k \\
2
\end{array}\right)-3}} n^{k-3}(1-\sigma) \leq\left|\mathscr{E}_{K_{k}^{(3)}}^{\mathscr{F}_{\mathcal{F}_{0}}}(h)\right| \leq \frac{\Pi_{B \in[k]^{3} \backslash\{1,2,3\}} \alpha_{B}}{\ell^{\left(\begin{array}{l}
k \\
2
\end{array}\right)-3}} n^{k-3}(1+\sigma) .
$$

With $\alpha_{B}=1$ for all $B \notin \mathscr{F}_{0}$, Theorem 10.12 is proved.

\section{THE EXTENSION LEMMA AND THE SPARSE-LINKS LEMMA}

In this section, we give a slight restatement of the Extension Lemma in terms of the upcoming so-called "Sparse-Links Lemma," Lemma 12.10. In this section, we develop the Sparse-Links Lemma and show that the Extension Lemma follows rather quickly from it. The Sparse-Links Lemma is difficult to prove and follows from some nontrivial tools developed in [13]. It is for this reason we choose to work in the upcoming technical language of [13].

The following remark gives the schedule and a partial strategy for the remaining parts of this paper.

Remark 12.1. The rest of this paper is organized as follows.

1. In Section 12.1, we present definitions needed to present the upcoming Sparse-Links Lemma, Lemma 12.10. We also present Lemma 12.10.

2. In Section 12.2, we confirm that the Sparse-Links Lemma easily implies the Extension Lemma.

3. In Section 13, we prove the Sparse-Links Lemma. This section involves a complex argument. 


\subsection{Definitions, Facts and the Sparse-Links Lemma}

We begin this section by defining an extended notion of regularity for cylinders. In the following, we abuse the Cartesian product notation for sets $A$ and $B$ by writing $A \times B=$ $\{\{a, b\}: a \in A, b \in B\}$.

Definition 12.2. Let $\gamma, \delta$ be positive reals, let $r$ be a positive integer and let $F$ be a bipartite graph with bipartition $(U, V)$. We say that $F$ is $(\gamma, \delta, r)$-regular if for any r-tuple of pairs of subsets $\left(\left\{U_{j}, V_{j}\right\}\right)_{j=1}^{r}, U_{j} \subseteq U, V_{j} \subseteq V, 1 \leq j \leq r$, satisfying

$$
\left|\bigcup_{j=1}^{r}\left(U_{j} \times V_{j}\right)\right|>\delta|U||V|,
$$

the following holds

$$
\frac{\left|F \cap \bigcup_{j=1}^{r}\left(U_{j} \times V_{j}\right)\right|}{\left|\bigcup_{j=1}^{r}\left(U_{j} \times V_{j}\right)\right|}>\gamma .
$$

Note that it follows directly from the definition that if $F$ is $(\gamma, \delta, r)$-regular, then $F$ is also $\left(\gamma, \delta^{\prime}, r^{\prime}\right)$-regular for any $\delta^{\prime} \geq \delta$ and positive integer $r^{\prime} \leq r$. We will use this fact repeatedly. Also, note that in (52), only a lower bound is considered. However, in application, Definition 12.2 is closely tied with the upcoming Definition 12.5 , where we will be concerned only with lower bounds of the quantity given in (52).

The following fact about $(\gamma, \delta, r)$ graphs is an easy consequence of Definition 12.2.

Fact 12.3. Let $\gamma, \delta$, and $r$ be given, and suppose $F$ is a bipartite graph with bipartition $(U, V)$. If $F$ is $(\gamma, \delta, r)$-regular, then all but $\delta|U|$ vertices $u \in U$ satisfy

$$
|N(u)|>\gamma|V|
$$

Typically, when we encounter $(\gamma, \delta, r)$-regular bipartite graphs, they will be $s u b$ graphs of other highly regular bipartite graphs. This situation prompts the following definition.

Definition 12.4. Let $\gamma, \delta, \epsilon$ be given positive reals, let $r, \ell$ be given positive integers, and let $F, G$ be two given bipartite graphs, each with bipartition $(U, V)$. We call the ordered pair of graphs $(F, G) a(\gamma, \delta, r, \ell, \epsilon)$-regular couple provided

(i) $F \subseteq G$,

(ii) $F$ is $(\gamma, \delta, r)$-regular,

(iii) $G$ is an $(\ell, \epsilon, 2)$-cylinder (i.e., a $\left(\frac{1}{\ell}, \epsilon\right)$-regular pair).

Note that in the definition above, $F$ being $(\gamma, \delta, r)$-regular only ensures a lower bound of $\gamma$ on the density described in (52). With $G$ being an $(\ell, \epsilon, 2)$-cylinder, Definition 2.1 
ensures that the density of a bipartite subgraph of $G$ on subsets $U^{\prime} \subseteq U,\left|U^{\prime}\right|>\epsilon|U|$, $V^{\prime} \subseteq V,\left|V^{\prime}\right|>\epsilon|V|$, is roughly $\frac{1}{\ell}$.

Related to Definition 12.4, the following environment is used pervasively throughout this section. Since we considered other environments earlier, we call the following Setup 2. For constants $k, \alpha, \delta, \ell, r, \epsilon$ and $n$, we consider the following situation for pairs of graphs $G$ and $F$.

\section{Setup 2.}

(a) $G=\bigcup_{1 \leq i<j \leq k} G^{i j}$ is an $(\ell, \epsilon, k)$-cylinder on $k$-partition $\left(V_{1}, \ldots, V_{k}\right),\left|V_{1}\right|$ $=\cdots=\left|V_{k}\right|=m$.

(b) $F=\bigcup_{1 \leq i<j \leq k} F^{i j}$ is a $k$-partite cylinder defined on the same $k$-partition $\left(V_{1}, \ldots\right.$, $\left.V_{k}\right)$.

(c) For each $\{i, j\} \in[k]^{2},\left(F^{i j}, G^{i j}\right)$ is an $\left(\frac{\alpha-2 \delta}{\ell}, \delta, r, \ell, \epsilon\right)$-regular couple.

The following definition relates to Setup 2. That following definition relates to Setup 2 will not be apparent until the upcoming Fact 12.6.

Definition 12.5. Let $\mathscr{H}$ be a k-partite 3-cylinder with $k$-partition $\left(V_{1}, \ldots, V_{k}\right)$, and let $G=\bigcup_{1 \leq i<j \leq k} G^{i j}$ be an underlying k-partite cylinder. Let $i, j, 1<i<j \leq k$, be integers and let $x \in V_{1}$. We define the $\{i, j\}$-link graph of $x, L_{x}^{i j}$, as

$$
L_{x}^{i j}=\left\{\{y, z\} \in G^{i j}\left[N_{G, i}(v), N_{G, j}(v)\right]:\{x, y, z\} \in \mathscr{H}\right\} .
$$

We further define the link graph of $x, L_{x}$, as

$$
L_{x}=\bigcup_{1<i<j \leq k} L_{x}^{i j}
$$

The following fact, given in Fact 4.9 of [12], connects Definition 12.5 to Setup 2. One can view this fact as partly projecting Setup 1 onto Setup 2 (cf. Remark 12.7).

Fact 12.6. For all $\alpha, \delta>0, \alpha>2 \delta$, and for all positive integers $k \geq 3, \ell$, and $r$, there exists $\epsilon>0$ so that whenever $n$ is sufficiently large and $\mathcal{H}$ and $\bigcup_{1 \leq i<j \leq k} G^{i j}$ satisfy the conditions of Setup 1 with the constants $k, \alpha, \delta, \ell, r, \epsilon$ and $n$, then

(a) all but $4(k-1) \epsilon n$ vertices $v \in V_{1}$ satisfy that, for each $i \in\{2, \ldots, k\}$,

$$
\frac{n}{\ell}(1-\epsilon) \leq\left|N_{G, i}(v)\right| \leq \frac{n}{\ell}(1+\epsilon),
$$

(b) all but $2\left(\begin{array}{c}k-1 \\ 2\end{array}\right) \sqrt{\delta} n$ vertices $v \in V_{1}$ satisfy that, for each $i, j, 1<i<j \leq k$, $\left(L_{v}^{i j}, G^{i j}\left[N_{G, i}(v), N_{G, j}(v)\right]\right)$ is an $\left(\frac{\alpha-2 \sqrt{\delta}}{\ell}, \sqrt{\delta}, \ell, r, 2 \ell \epsilon\right)$-regular couple.

Remark 12.7. Observe in Fact 12.6 that the graph $L_{v}=\bigcup_{1<i<j \leq k} L_{v}^{i j}$ plays the role of "F" in Setup 2 and the graph $\bigcup_{1<i<j \leq k} G^{i j}\left[N_{G, i}(v), N_{G, j}(v)\right]$ plays the role of " $G$ " in Setup 2. Note, as well, the "asymptotic" quantity " $n / \ell$ " plays the role of $m$ in Setup 2. 
Thus, Fact 12.6 asserts that in the context of Setup 1, with appropriate constants, most vertices $v \in V_{1}$ give rise to graphs $L_{v}$ and $\bigcup_{1<i<j \leq k} G^{i j}\left[N_{G, i}(v), N_{G, j}(v)\right]$ satisfying the conditions of Setup 2.

Fact 12.6 prompts the following definition.

Definition 12.8. A vertex $v \in V_{1}$ is said to be a good vertex if it satisfies

(a) for each $i \in\{2, \ldots, k\}$,

$$
\frac{n}{\ell}(1-\epsilon) \leq\left|N_{G, i}(v)\right| \leq \frac{n}{\ell}(1+\epsilon),
$$

(b) for all $i, j, 1<i<j \leq k$, ( $\left.L_{v}^{i j}, G^{i j}\left[N_{G, i}(v), N_{G, j}(v)\right]\right)$ is an $\left(\frac{\alpha-2 \sqrt{\delta}}{\ell}\right.$, $\sqrt{\delta}, \ell, \mathrm{r}, 2 \ell \epsilon$ )-regular couple.

By Fact 12.6, we see almost all vertices $v \in V_{1}$ are good vertices.

The following definition addresses the thesis of the Sparse-Links Lemma, Lemma 12.10 .

Definition 12.9. For positive $\sigma$ and a good vertex $v \in V_{1}$, a link edge $e=\{x, y\} \in L_{v}^{23}$ is said to be $\sigma$-sparse with respect to $v$ if the triple $\{v, x, y\} \in \mathcal{H}$ satisfies

$$
\left|\mathscr{E}_{K_{k}^{(3)}}(\{v, x, y\})\right| \leq \frac{\alpha^{\left(\begin{array}{l}
k \\
3
\end{array}\right)-1}}{\ell\left(\begin{array}{l}
k \\
2
\end{array}\right)-3} n^{k-3}(1-\sigma)
$$

(cf. Definition 10.11). In other words, if $\{v, x, y\} \in \mathcal{H}$ is contained in no more than $\frac{\alpha\left(\begin{array}{l}k \\ 3\end{array}\right)-1}{\ell\left(\begin{array}{l}k \\ 2\end{array}\right)-3} n k-3(1-\sigma)$ copies of $K_{k}^{(3)}$.

We now present the Sparse-Links Lemma.

Lemma 12.10 (Sparse-Links Lemma). Let $k \geq 5$ be an integer. For all positive reals $\alpha$, $\beta, \sigma$, and $\gamma$, there exists $\delta>0$ such that, for all $\ell>\frac{1}{\delta}$, there exists $r$ and $\epsilon$ so that whenever $n$ is sufficiently large and $\mathcal{H}$ and $G=\bigcup_{1 \leq i<j \leq k} G^{i j}$ satisfy the hypothesis of Setup 1 with the constants $k, \alpha, \delta, \ell, r, \epsilon$, and $n$ and 3 -uniform hypergraph $\mathscr{f}_{0}=K_{k}^{(3)}$, then, for all but fewer than $\beta n$ good vertices $v \in V_{1}$, the number of link edges of $v$ in $L_{v}^{23}$ which are $\sigma$-sparse with respect to $v$ is less than $\gamma_{\bar{\ell}}^{\alpha}\left(\frac{n}{\ell}(1+\epsilon)\right)^{2}$.

\subsection{Proof of Lemma 10.13}

In this section, we use Lemma 12.10 to prove Lemma 10.13 . We begin by defining the promised constants.

Definitions of the Constants. Let $k \geq 5$ be a given integer, and let $\alpha, \mu, \sigma$ be given positive reals. Define auxiliary positive constants $\beta, \gamma, \mu_{1}$ so that 


$$
2 \beta+2 \gamma<\frac{\mu_{1} \alpha}{2}<\frac{\mu \alpha}{8} .
$$

Let

$$
\delta_{12.10}=\delta_{12.10}(\alpha, \beta, \sigma, \gamma)
$$

be that constant guaranteed by Lemma 12.10 . Set $\delta>0$ to satisfy

$$
\begin{aligned}
\delta & \leq \delta_{12.10}, \\
2 k^{2} \sqrt{\delta} & \leq \frac{\mu_{1} \alpha}{4} .
\end{aligned}
$$

Let $\ell>\frac{1}{\delta}$ be a given integer. Let

$$
r=r_{12.10}\left(\alpha, \beta, \sigma, \gamma, \delta_{12.10}, \ell\right)
$$

be that constant guaranteed by Lemma 12.10 .

Let

$$
\epsilon_{12.10}=\epsilon_{12.10}\left(\alpha, \beta, \sigma, \gamma, \delta_{12.10}, \ell, r_{12.10}\right)
$$

be that constant guaranteed by Lemma 12.10 . Let

$$
\epsilon_{12.6}=\epsilon_{12.6}(\alpha, \delta, \ell, r)
$$

be that constant guaranteed by Fact 12.6. Set $\epsilon>0$ to satisfy

$$
\epsilon \leq \min \left\{\frac{\mu_{1} \alpha}{4 k \ell^{3}}, \epsilon_{12.10}, \epsilon_{12.6}\right\} .
$$

This ends our definitions of the constants.

Proof of Lemma 10.13. Suppose $k \geq 5$ is a given integer, and let $\alpha, \mu, \sigma$, be given positive reals. Let $\delta>0$ be given by (56) and (57). Let $\ell>\frac{1}{\delta}$ be a given integer, and let $r$ and $\epsilon$ be given in (58) and (61) respectively. Let $\mathcal{H}$ be a $k$-partite 3 -cylinder and $G$ $=\bigcup_{1 \leq i<j \leq k} G^{i j}$ an underlying cylinder of $\mathscr{H}$ which together satisfy the conditions of Setup 1 with constants $k, \alpha, \delta, \ell, r, \epsilon$, and $n$, where $n$ is sufficiently large, and triple system $\mathscr{F}_{0}=K_{k}^{(3)}$. We show that all but $\mu|\mathscr{H}(\{1,2,3\})|$ triples $h \in \mathscr{H}(\{1,2,3\})$ belong to at least

$$
\frac{\alpha^{\left(\begin{array}{l}
k \\
3
\end{array}\right)-1}}{\ell^{\left(\begin{array}{l}
k \\
2
\end{array}\right)-3}} n^{k-3}(1-\sigma)
$$

copies of $K_{k}^{(3)}$. 
We define a set $\mathscr{H}^{\prime} \subseteq \mathscr{H}(\{1,2,3\})$ so that $\left|\mathscr{H}^{\prime}\right|<\mu|\mathscr{H}(\{1,2,3\})|$ and so that every triple $h \in \mathscr{H}(\{1,2,3\}) \backslash \mathcal{H}^{\prime}$ belongs to at least

$$
\frac{\alpha^{\left(\begin{array}{l}
k \\
3
\end{array}\right)-1}}{\ell^{\left(\begin{array}{l}
k \\
2
\end{array}\right)-3}} n^{k-3}(1-\sigma)
$$

copies of $K_{k}^{(3)}$. Consider the following sets:

(i) Let $S_{0} \subseteq V_{1}$ be the set of all good vertices (cf. Definition 12.8). We determine the number of triples $h \in \mathscr{H}(\{1,2,3\})$ which are incident to vertices in $V_{1} \backslash S_{0}$ by using Fact 12.6. Indeed, by our choice of $\epsilon$ in (61), $\epsilon \leq \epsilon_{12.6}(\alpha, \delta, \ell, r)$, thus, we may apply Fact 12.6 to $\mathscr{H}$ and $G=\bigcup_{1 \leq i<j \leq k} G^{i j}$ with the constants $k, \alpha, \delta, \ell, r, \epsilon$, and $n$.

(a) Let $S_{1} \subseteq V_{1}$ be the set of all vertices $v \in V_{1}$ such that

$$
\frac{n}{\ell}(1-\epsilon)<\left|N_{G, i}(v)\right|<\frac{n}{\ell}(1+\epsilon)
$$

for all $i=2, \ldots, k$. Note that by statement (a) of Fact 12.6, $\left|S_{1}\right|>(1-4 k \epsilon) n$. Let $\mathcal{H}_{1}$ be the set of all triples $h \in \mathcal{H}(\{1,2,3\})$ such that there exists a vertex $v \in V_{1} \backslash S_{1}$ with $v \in h$. Note that

$$
\left|\mathscr{H}_{1}\right|<4 k \epsilon n^{3} .
$$

(b) Let $S_{2} \subseteq S_{1}$ be the set of all vertices $v \in S_{1}$ such that for all $i, j, 1<i<j \leq$ $k,\left(L_{v}^{i j}, G^{i j}(v, G)\right)$ is an $\left(\frac{\alpha-2 \sqrt{\delta}}{\ell}, \sqrt{\delta}, \ell, r, 2 \ell \epsilon\right)$-regular couple. We conclude from statement (b) of Fact 12.6 that $\left|S_{2}\right|>\left(1-k^{2} \sqrt{\delta}\right) n$. Let $\mathscr{H}_{2}$ be the set of all triples $h \in \mathscr{H}(\{1,2,3\})$ such that there exists a vertex $v \in S_{1} \backslash S_{2}$ with $v \in h$. Note that since each $v \in S_{1} \backslash S_{2}$ satisfies (62), each such vertex could be contained in at most $\frac{1}{\ell}(1+\epsilon)\left(\frac{n}{\ell}(1+\epsilon)\right)^{2}$ triples $h \in \mathcal{H}(\{1,2,3\})$. Thus,

$$
\left|\mathscr{H}_{2}\right|<k^{2} \sqrt{\delta} n\left(\frac{n}{\ell}(1+\epsilon)\right)^{2}\left(\frac{1}{\ell}(1+\epsilon)\right)<2 k^{2} \sqrt{\delta} \frac{n^{3}}{\ell^{3}} .
$$

Note that, by (63) and (64), the number of triples $h \in \mathscr{H}(\{1,2,3\})$ which are incident to vertices in $V_{1} \backslash S_{0}$ is no more than $\left(4 k \epsilon \ell^{3}+2 k^{2} \sqrt{\delta}\right) \frac{n^{3}}{\ell^{3}}$.

(ii) Let $\mathscr{H}_{3}$ be the set of all triples $h=\{v, x, y\} \in \mathscr{H}(\{1,2,3\})$ such that $v \in S_{2}$ and $\{x, y\} \in L_{v}^{23}$ is $\sigma$-sparse with respect to $v$ (cf. Definition 12.9). By our choice of constants $\delta \leq \delta_{12.10}(\alpha, \beta, \sigma, \gamma)$ in (55), $r=r_{12.10}\left(\alpha, \beta, \sigma, \gamma, \delta_{12.10}, \ell\right)$ in (58) and $\epsilon \leq \epsilon_{12.10}\left(\alpha, \beta, \sigma, \gamma, \delta_{12.10}, \ell, r_{12.10}\right)$ in (59), we may apply Lemma 12.10 to infer that, for all but $\beta n$ vertices $v \in S_{2}$, no more than $\gamma \frac{\alpha}{\ell}\left(\frac{n}{\ell}(1+\epsilon)\right)^{2}$ of the links of $v$ are $\sigma$-sparse with respect to $v$. Note that since each $v \in S_{2}$ satisfies (62), the number of triples which are incident to any fixed vertex $v$ of the exceptional $\beta n$ vertices is at most $\frac{1}{\ell}(1+\epsilon)\left(\frac{n^{3}}{\ell}(1+\epsilon)\right)^{2}<472 \frac{n^{2}}{\ell^{3}}$. Thus, we conclude

$$
\left|\mathscr{H}_{3}\right|<2 \beta \frac{n^{3}}{\ell^{3}}+\left|S_{2}\right| \gamma \frac{\alpha}{\ell}\left(\frac{n}{\ell}(1+\epsilon)\right)^{2}<2 \beta \frac{n^{3}}{\ell^{3}}+2 \alpha \gamma \frac{n^{3}}{\ell^{3}} .
$$


Set $\mathscr{H}^{\prime}=\mathscr{H}_{1} \cup \mathscr{H}_{2} \cup \mathscr{H}_{3}$. Then due to (63)-(65), we conclude

$$
\left|\mathscr{H}^{\prime}\right|<k \epsilon n^{3}+2 k^{2} \sqrt{\delta} \frac{n^{3}}{\ell^{3}}+2 \beta \frac{n^{3}}{\ell^{3}}+2 \gamma \frac{n^{3}}{\ell^{3}}=\left(k \epsilon \ell^{3}+2 k^{2} \sqrt{\delta}+2 \beta+2 \gamma\right) \frac{n^{3}}{\ell^{3}} .
$$

By the inequalities in (54), (57), and (61), we infer

$$
\left|\mathscr{H}^{\prime}\right|<\mu_{1} \alpha \frac{n^{3}}{\ell^{3}}<\mu \frac{\alpha}{4} \frac{n^{3}}{\ell^{3}}
$$

As $\mathscr{H}(\{1,2,3\})$ is an $(\alpha, \delta, r)$-regular triad, we conclude that $|\mathscr{H}(\{1,2,3\})|>(\alpha-$ $\delta) \mid \mathscr{K}_{3}(G(\{1,2,3\}) \mid$. Using Fact 10.3, we further conclude that

$$
|\mathscr{H}(\{1,2,3\})|>(\alpha-\delta) \frac{1}{2} \frac{n^{3}}{\ell^{3}}>\frac{\alpha}{4} \frac{n^{3}}{\ell^{3}} .
$$

Thus, from (66), we conclude

$$
\left|\mathscr{H}^{\prime}\right|<\mu \frac{\alpha}{4} \frac{n^{3}}{\ell^{3}}<\mu|\mathscr{H}(\{1,2,3\})| .
$$

Any triple $h=\{v, x, y\} \in \mathscr{H}_{\mathcal{H}} \mathscr{H}^{\prime}$ must be such that $v \in S_{2}$ and $\{x, y\} \in L_{v}^{23}$ is not $\sigma$-sparse with respect to $v$. Thus, by Definition 12.9 the number of copies of $K_{k}^{(3)}$ containing $h \in \mathscr{H} \backslash \mathcal{H}^{\prime}$ is at least

$$
\frac{\alpha^{\left(\begin{array}{l}
k \\
3
\end{array}\right)-1}}{\ell^{\left(\begin{array}{l}
k \\
2
\end{array}\right)-3}} n^{k-3}(1-\sigma)
$$

\section{PROOF OF THE SPARSE-LINKS LEMMA}

We begin with the following elementary definitions and facts we need for our proof of the Sparse-Links Lemma.

\subsection{Elementary Definitions and Facts}

We return to the context of Setup 2 and establish some helpful notation. For a pair of $k$-partite graphs $F=\bigcup_{1 \leq i<j \leq k} F^{i j}$ and $G=\bigcup_{1 \leq i<j \leq k} G^{i j}$ with common $k$-partition $\left(V_{1}, \ldots, V_{k}\right)$, suppose that $F^{i j} \subseteq G^{i j}$ for all $i, j, 1 \leq i<j \leq k$. For $v \in V_{1}, H \in\{F$, $G\}$, define

$$
F^{i j}(v, H)=\left\{\{x, y\} \in F^{i j}: x \in N_{H, i}(v), y \in N_{H, j}(v)\right\}=F^{i j}\left[N_{H, i}(v), N_{H, j}(v)\right]
$$

and 


$$
G^{i j}(v, H)=\left\{\{x, y\} \in G^{i j}: x \in N_{H, i}(v), y \in N_{H, j}(v)\right\}=G^{i j}\left[N_{H, i}(v), N_{H, j}(v)\right] .
$$

Similarly, for $e=\left\{v_{1}, v_{2}\right\} \in F^{12}$, for a fixed pair $i, j, 2<i<j \leq k$, define the graphs $F^{i j}(e, F)$ and $G^{i j}(e, F)$ as

$$
F^{i j}(e, F)=F^{i j}\left(v_{1}, F\right) \cap F^{i j}\left(v_{2}, F\right)
$$

and

$$
G^{i j}(e, F)=G^{i j}\left(v_{1}, F\right) \cap G^{i j}\left(v_{2}, F\right),
$$

respectively.

Our first fact essentially states that when a pair of graphs $F, G$ satisfies the conditions of Setup 2, then so will the graphs induced by the neighborhoods of almost all of their vertices. More precisely, we have the following fact.

Fact 13.1. For all integers $k \geq 3$ and $\alpha, \delta_{0}>0$, there exists $\delta>0$, so that, for all integers $\ell>\frac{1}{\delta}$ and positive integers $r_{0}$, there exists an integer $r$ so that for all $\epsilon_{0}>0$ there exists $\epsilon>0$ so that whenever $m$ is sufficiently large and a pair of graphs $F$ and $G$ satisfy the conditions of Setup 2 with parameters $k, \alpha, \delta, \ell, r, \epsilon$, and $m$, then all but $\left(\begin{array}{c}k-1 \\ 2\end{array}\right)\left(2 \epsilon+2 \delta+\frac{4 \delta \ell^{2} \epsilon}{\delta_{0}(\alpha-2 \delta)^{2}}\right) m$ vertices $v \in V_{1}$ satisfy

(a) for each $1<i \leq k,\left|N_{F, i}(v)\right| \geq \frac{\alpha-2 \delta}{\ell} m$ and $\frac{m}{\ell}(1-\epsilon) \leq\left|N_{G, i}(v)\right| \leq \frac{m}{\ell}(1+\epsilon)$,

(b) for all $1<i<j \leq k,\left(F^{i j}(v, F), G^{i j}(v, F)\right)$ is an $\left(\frac{\alpha-2 \delta_{0}}{\ell}, \delta_{0}, \ell, r_{0}, \epsilon_{0}\right)$-regular couple.

We mention that Fact 13.1 was given in Fact 4.6 in [12].

The following statement complements Fact 13.1. While according to Fact $13.1,\left(F^{i j}(v\right.$, $\left.F), G^{i j}(v, F)\right), 1<i<j \leq k$, is a regular couple for a typical vertex $v \in V_{1}$, Proposition 13.2 asserts that $\left(F^{i j}(e, F), G^{i j}(e, F)\right), 2<i<j \leq k$, is a regular couple for a "typical edge" $e \in F^{12}$. Moreover, the sizes $\left|N_{F, i}(e)\right|$ and $\left|N_{G, i}(e)\right|$ are "correct." More precisely, our proposition states the following.

Proposition 13.2. Let $k \geq 4$ be an integer. For all reals $\alpha, \delta_{0}, \beta>0$, there exists $\delta>$ 0 so that for all integers $\ell>\frac{1}{\delta}$ and all positive integers $r_{0}$, there exists an integer $r$ so that for all $\epsilon_{0}>0$, there exists $\epsilon>0$ so that whenever $m$ is sufficiently large and $F$ and $G$ satisfy Setup 2 with constants $k, \alpha, \delta, \ell, r, \epsilon$, and $m$, then all but $\beta\left|F^{12}\right|$ edges $e \in F^{12}$ satisfy

(a) for each $i \in\{3, \ldots, k\}$,

and

$$
\left|N_{F, i}(e)\right| \geq\left(\frac{\alpha-2 \delta}{\ell}\right)^{2} m
$$

$$
\frac{m}{\ell^{2}}(1-\epsilon)^{2} \leq\left|N_{G, i}(e)\right| \leq \frac{m}{\ell^{2}}(1+\epsilon)^{2},
$$


(b) for all $i, j, 2<i<j \leq k,\left(F^{i j}(e, F), G^{i j}(e, F)\right)$ is an $\left(\frac{\alpha-2 \delta_{0}}{\ell}, \delta_{0}, \ell, r_{0}, \epsilon_{0}\right)$ regular couple.

Unlike Fact 13.1, Proposition 13.2 did not appear in any earlier papers, and thus we state it as a proposition. However, Proposition 13.2 can be proved by a double application of Fact 13.1. We therefore omit the standard argument.

In the upcoming Remark 13.4, we explain how the following definition is motivated by Fact 12.6, Definition 12.8 and Proposition 13.2.

Definition 13.3. For a good vertex $v \in V_{1}$, and constants $\delta_{0}, r_{0}$ and $\epsilon_{0}$, a link edge $e \in$ $L_{v}^{23}$ is said to be a good link edge with respect to $\delta_{0}, r_{0}$, and $\epsilon_{0}$ provided

(a) for each $i \in\{4, \ldots, k\}$,

$$
\left|N_{L_{v}, i}(e)\right| \geq\left(\frac{\alpha-2 \sqrt{\delta}}{\ell}\right)^{2} \frac{n}{\ell}(1-\epsilon)
$$

(b) for all $i, j, 3<i<j \leq k$, (L $\left.L_{v}^{i j}\left(e, L_{v}\right), G_{v}^{i j}\left(e, L_{v}\right)\right)$ is an $\left(\frac{\alpha-2 \delta_{0}}{\ell}, \delta_{0}, \ell\right.$, $\mathrm{r}_{0}, \epsilon_{0}$ )-regular couple.

Remark 13.4. While we prove it precisely later in context (cf. Claim 13.11), we mention that for each fixed good vertex $v \in V_{1}$, nearly all link edges $e \in L_{v}^{23}$ are good link edges.

Indeed, by Definition 12.8, part (a) all $\left|N_{G, i}(v)\right| \sim n / \ell, 1<i \leq k$. As in Remark 12.7, we set $m \sim n / \ell$ (as in Setup 2). Similarly we set $L_{v}=\bigcup_{1<i<j \leq k} L_{v}^{i j}$ to play the role of " $F$ " and $\bigcup_{1<i<j \leq k} G^{i j}(v, G)$ to play the role of " $G$." In view of Proposition 13.2, part (a), most edges $e \in L_{v}^{23}=F^{12}$ satisfy

$$
\left|N_{L_{v}, i}(e)\right| \geq\left(\frac{\alpha-2 \delta}{\ell}\right)^{2} m \sim\left(\frac{\alpha-2 \delta}{\ell}\right)^{2} \frac{n}{\ell} .
$$

Thus, part (a) in Definition 13.3 is satisfied.

By Definition 12.8, part (b) all $\left(L_{v}^{i j}, G^{i j}(v, G)\right), 1<i<j \leq k$, are "regular" couples. In view of Proposition 13.2, part (b), most edges $e \in L^{23}$ satisfy (b) of Definition 13.3.

Our next fact was given in Fact 4.7 of [12] and is related to Fact 10.3.

Fact 13.5. For all integers $k \geq 3$ and all $\alpha, \beta>0$, there exists $\delta>0$ so that, for all integers $\ell \geq \frac{1}{\delta}$, there exist an integer $r$ and $\epsilon>0$ so that whenever $m$ is sufficiently large and graphs $F$ and $G$ satisfy Setup 2 with constants $k, \alpha, \delta, \ell, r, \epsilon$, and $n$, then

$$
\left|\mathscr{K}_{k}(F)\right| \geq\left(\frac{\alpha}{\ell}\right)^{\left(\begin{array}{l}
k \\
2
\end{array}\right)} m^{k}(1-\beta) .
$$

The following fact appeared as Fact 4.2 in [12]. This fact is more basic than the previous facts of this subsection. 
Fact 13.6. Let $k, r, \ell$ be given integers, and let $\epsilon>0$ and $\beta>0$ be given real numbers. Suppose $G=\bigcup_{0 \leq i<j \leq k} G^{i j}$ is a k-partite cylinder with $k$-partition $\left(W_{0}, W_{1}, \ldots, W_{k-1}\right)$, $\left|W_{1}\right|=\cdots=\left|W_{k-1}\right|=n$, and $\left|W_{0}\right|=\beta n$, where $n$ is assumed to be sufficiently large. Suppose $G$ satisfies that for all $j \in[k-1]$,

(i) the subgraph $G^{0 j}$ of $G$ induced by $W_{0} \cup W_{j}$ satisfies that whenever $W_{0}^{\prime} \subseteq W_{0}$ and $W_{j}^{\prime} \subseteq W_{j},\left|W_{0}^{\prime}\right| \geq \epsilon n$ and $\left|W_{j}^{\prime}\right| \geq \epsilon n$, then

$$
\frac{1}{\ell}(1-\epsilon)<d_{G^{0 j}}\left(W_{0}^{\prime}, W_{j}^{\prime}\right)<\frac{1}{\ell}(1+\epsilon),
$$

(ii) for all $x \in W_{0},\left|N_{j}(x)\right| \leq \frac{n}{\ell}(1+\epsilon)$.

Then there exist pairwise disjoint r-element subsets $D_{1}, \ldots, D_{q}, q=\left\lceil\frac{\beta n}{r}(1-r k \epsilon / \beta)\right\rceil$, satisfying

(a) for each $i \in[q], D_{i}=\left\{x_{1}^{(i)}, \ldots, x_{r}^{(i)}\right\} \subseteq W_{0}$,

(b) for each $i \in[q]$, for all $\{u, v\} \in[r]^{2}$, and for all $j \in[k-1]$,

$$
\left|N_{j}\left(x_{u}^{(i)}\right) \cap N_{j}\left(x_{v}^{(i)}\right)\right| \leq \frac{n}{\ell^{2}}(1+\epsilon)^{2} .
$$

\subsection{Advanced Definitions and a Technical Lemma from [13]}

In this subsection, we present a lemma proved in [13] which will help us prove the Sparse-Links Lemma. This auxiliary lemma, Lemma 13.10, is difficult and has a highly nontrivial proof. For more about the proof of Lemma 13.10, see [13].

Before we may state Lemma 13.10, we state some definitions. We begin by extending our notion of density in a hypergraph to a type of clique-density.

Definition 13.7. Let $\mathcal{H}$ be a k-partite 3-cylinder with underlying k-partite cylinder $G$ $=\bigcup_{1 \leq i<j \leq k} G^{i j}$, and let $B \subseteq[k],|B|=b$. For the $B$-cylinder $G(B)$, we define the density $d_{\mathscr{H}}(G(B))$ of $\mathscr{H}_{\text {with }}$ respect to the B-cylinder $G(B)$ as

$$
d_{\mathscr{H}}(G(B))= \begin{cases}\frac{\left|\mathscr{K}_{b}(\mathscr{H}(B)) \cap \mathscr{K}_{b}(G(B))\right|}{\left|\mathscr{K}_{b}(G(B))\right|} & \text { if }\left|\mathscr{K}_{b}(G(B))\right|>0 \\ 0 & \text { otherwise. }\end{cases}
$$

In other words, this density counts the proportion of copies of $K_{b}^{(2)}$ in $G(B)$ which underlie copies of $K_{b}^{(3)}$ in $\mathscr{H}(B)$.

More generally, let $Q_{B} \subseteq G(B), B \subseteq[k],|B|=b$, where $Q_{B}=\bigcup_{\{i, j\} \in[B]^{2}} Q_{B}^{i j}$. One can define the density $d_{\mathscr{H}}\left(Q_{B}\right)$ of $\mathcal{H}$ with respect to $Q_{B}$ as

$$
d_{\mathscr{H}}\left(Q_{B}\right)= \begin{cases}\frac{\left|\mathscr{K}_{b}(\mathscr{H}) \cap \mathscr{K}_{b}\left(Q_{B}\right)\right|}{\left|\mathscr{K}_{b}\left(Q_{B}\right)\right|} & \text { if }\left|\mathscr{K}_{b}\left(Q_{B}\right)\right|>0 \\ 0 & \text { otherwise. }\end{cases}
$$

For our purposes, we will need an extension of the definition above, and will consider a 
simultaneous density of $\mathscr{H}$ with respect to a fixed $r$-tuple of $b$-partite cylinders $\left(Q_{B}(1), \ldots, Q_{B}(r)\right)$.

Definition 13.8. Let $\mathscr{H}$ be a k-partite 3-cylinder with underlying $k$-partite cylinder $G$ $=\bigcup_{1 \leq i<j \leq k} G^{i j}$, and let $B \subset[k],|B|=b$. Let $\vec{Q}=\vec{Q}_{B}=\left(Q_{B}(1), \ldots, Q_{B}(r)\right)$ be an $r$-tuple of $B$-cylinders $Q_{B}(s)=\bigcup_{\{i, j\} \in[B]^{2}} Q_{B}^{i j}(s)$ satisfying that for every $s \in[r],\{i, j\} \in$ $[B]^{2}, Q_{B}^{i j}(s) \subseteq G^{i j}$. We define the density $d_{\mathscr{H}}(\vec{Q})$ of $\vec{Q}$ as

$$
d_{\mathscr{H}}(\vec{Q})= \begin{cases}\frac{\left|\mathscr{K}_{b}(\mathscr{H}) \cap \bigcup_{s=1}^{r} \mathscr{K}_{b}\left(Q_{B}(s)\right)\right|}{\left|\bigcup_{s=1}^{r} \mathscr{K}_{b}\left(Q_{B}(s)\right)\right|} & \text { if }\left|\bigcup_{s=1}^{r} \mathscr{K}_{b}\left(Q_{B}(s)\right)\right|>0 \\ 0 & \text { otherwise. }\end{cases}
$$

We now give a definition which provides an extended notion of regularity for 3-cylinders. The reader is advised that the following concepts may be the most complicated notions in this paper.

Definition 13.9. Let $\mathscr{H}$ be a k-partite 3-cylinder, and suppose $G=\bigcup_{1 \leq i<j \leq k} G^{i j}$ is an underlying cylinder of $\mathcal{H}$. Let $B \subseteq[k],|B|=b, r$ and $\delta>0$ be given. We say that the $B$-3-cylinder $\mathscr{H}(B)$ is $(\delta, r)$-regular with respect to $G(B)$ if the following regularity condition is satisfied: Suppose $\vec{Q}=\vec{Q}_{B}=\left(Q_{B}(1), \ldots, Q_{B}(r)\right)$ is an r-tuple of B-cylinders $Q_{B}(s)=\bigcup_{\{i, j\} \in[B]^{2}} Q_{B}^{i j}(s)$ satisfying that for all $s \in[r]$, and all $\{i, j\} \in[B]^{2}, Q_{B}^{i j}(s) \subseteq$ $G^{i j}$. Then $\left|\bigcup_{s=1}^{r} \mathscr{K}_{b}\left(Q_{B}(s)\right)\right|>\delta\left|\mathscr{K}_{b}(G(B))\right|$ implies

$$
d_{\mathscr{H}}(\vec{Q})>d_{\mathscr{H}}(G(B))-\delta
$$

If, moreover, it is specified that $\mathscr{H}(B)$ is $(\delta, r)$-regular with respect to $G(B)$ with density $d_{\mathscr{H}}(G(B)) \geq \alpha-\delta$ for some $\alpha$, then we say that the $B$-3-cylinder $\mathscr{H}(B) i s(\alpha, \delta, r)$-regular with respect to $G(B)$.

Note that, for $k=3$, the above definition reduces to the concept of $(\delta, r)$-regularity in Definition 2.8. In that case, this definition roughly asserts that graph triangles formed by the edges of $G$ are matched by triples of $\mathcal{H}$ in at least an $\alpha-\delta$ portion. For $k>3$ and $B=[k]$, this definition roughly states that instead of graph triangles, graph cliques $K_{k}^{(2)}$ underlie hypergraph cliques $K_{k}^{(3)}$ in $\mathscr{H}$ in at least a "correct" portion.

The following nontrivial technical lemma was proved in [12] (cf. Statement 3.5) and [13]. It states that the seemingly weaker concept of $(\delta, r)$-regularity for $k=3$ ensures the validity of the stronger concept above for $k>3$ with adapted constants.

Lemma 13.10. Let $k \geq 3$ be an integer. For all positive $\alpha$ and $\delta_{k}$, there exists $\delta>0$ so that, for all integers $\ell \geq \frac{1}{\delta}$, for all positive integers $r_{k}$, there exist $r, \epsilon$ so that whenever $n$ is sufficiently large and k-partite 3-cylinder $\mathscr{H}$ and graph $G$ satisfy Setup 1 with constants $\alpha, \delta, \ell, r, \epsilon$, and $n$ and 3-uniform hypergraph $\mathscr{F}_{0}=K_{k}^{(3)}$, then $\mathscr{H}=\mathscr{H}([k])$ is $\left(\alpha\left(\begin{array}{l}k \\ 3\end{array}\right), \delta_{k}, r_{k}\right)$-regular with respect to $G=G([k])$. 


\subsection{Proof of Lemma 12.10}

Before giving the proof of Lemma 12.10, we give the definitions of the constants involved. We remark that the definitions of the constants guaranteed by Lemma 12.10 are quite technical. For the reader not interested in these details, we cite the following hierarchy governing the sizes of these constants:

$$
\alpha, \beta, \sigma, \gamma \gg \delta>\frac{1}{\ell}, \frac{1}{r} \gg \epsilon
$$

Definitions of the Constants. Let $k \geq 5$ be a given integer, and let $\alpha, \beta, \sigma$, and $\gamma$ be given positive constants. Without loss of generality, assume $\sigma<\frac{1}{4}$.

Definition of $\boldsymbol{\delta}$. We first choose auxiliary constants $\tau>0$ and $\beta^{\prime}>0$ such that

$$
\begin{gathered}
(1-\tau)^{7} \geq 1-\sigma^{2} \geq \frac{1}{2}, \\
1-\frac{2 \beta^{\prime}}{\alpha \gamma}>1-\tau .
\end{gathered}
$$

Let

$$
\delta_{13.5}=\delta_{13.5}(\alpha, \tau)
$$

be that constant guaranteed by Fact 13.5. Let

$$
\delta_{13.2}=\delta_{13.2}\left(\alpha, \delta_{13.5}, \beta^{\prime}\right)
$$

be that constant guaranteed by Proposition 13.2.

Choose $\delta_{k-1}>0$ to satisfy

$$
\begin{gathered}
\frac{8 \delta_{k-1}}{\alpha\left(\begin{array}{c}
k-1 \\
2
\end{array}\right) \gamma}<\min \left\{\frac{\gamma}{8} \alpha\left(\begin{array}{c}
k-1 \\
2
\end{array}\right), \tau\right\}, \\
1-\frac{2 \delta_{k-1}}{\alpha\left(\begin{array}{c}
k-1 \\
3
\end{array}\right)}>1-\tau,
\end{gathered}
$$

and such that by setting

$$
\zeta=\frac{8 \delta_{k-1}}{\alpha\left(\begin{array}{c}
k-1 \\
2
\end{array}\right) \gamma},
$$

the following holds 


$$
1-\frac{2 \zeta}{\gamma \alpha\left(\begin{array}{c}
k-1 \\
2
\end{array}\right)(1-\tau)^{4}}>1-\tau .
$$

Let

$$
\delta_{13.10}=\delta_{13.10}\left(\alpha, \delta_{k-1}\right)
$$

be that constant guaranteed by Lemma 13.10 . Set $\delta>0$ to satisfy

$$
\begin{gathered}
\delta \leq \min \left\{\delta_{13.2}^{2}, \delta_{13.10}\right\} \\
\left(1-\frac{2 \sqrt{\delta}}{\alpha}\right)^{2(k-3)+1}>1-\tau .
\end{gathered}
$$

This concludes our definition of $\delta$.

Let $\ell>\frac{1}{\delta}$ be a given integer. We now define the promised constants $r$ and $\epsilon$.

Definitions of $\mathbf{r}$ and $\boldsymbol{\epsilon}$. Let

$$
r_{13.5}=r_{13.5}\left(\alpha, \tau, \delta_{13.5}, \ell\right)
$$

be that constant guaranteed by Fact 13.5. Let

$$
r_{13.2}=r_{13.2}\left(\alpha, \delta_{13.5}, \beta^{\prime}, \delta_{13.2}, \ell, r_{13.5}\right)
$$

be that constant guaranteed by Proposition 13.2. For $\zeta$ given in (74), set

$$
r_{k-1}=\zeta \ell^{k-1}
$$

Let

$$
r_{13.10}=r_{13.10}\left(\alpha, \delta_{k-1}, \delta_{13.10}, \ell, r_{k-1}\right)
$$

be that constant guaranteed by Lemma 13.10. Set

$$
r=\max \left\{r_{13.5}, r_{13.2}, r_{13.10}\right\}
$$

Let

$$
\epsilon_{13.5}=\epsilon_{13.5}\left(\alpha, \tau, \delta_{13.5}, \ell, r_{13.5}\right)
$$

be that constant guaranteed by Fact 13.5. Let

$$
\epsilon_{13.2}=\epsilon_{13.2}\left(\alpha, \delta_{13.5}, \beta^{\prime}, \delta_{13.2}, \ell, r_{13.5}, r_{13.2}, \epsilon_{13.5}\right)
$$


be that constant guaranteed by Proposition 13.2. Let

$$
\epsilon_{13.10}=\epsilon_{13.10}\left(\alpha, \delta_{k-1}, \delta_{13.10}, \ell, r_{k-1}, r_{13.10}\right)
$$

be that constant guaranteed by Lemma 13.10. For the constant $\theta=1$, let

$$
\epsilon_{10.3}=\epsilon_{10.3}(k-1,1, \ell)
$$

be that constant guaranteed by Fact 10.3. Let $\epsilon^{\prime}>0$ satisfy

$$
\begin{gathered}
\left(1+\epsilon^{\prime}\right)\left(\begin{array}{c}
k+1 \\
2
\end{array}\right)<2, \\
1-\frac{r_{k-1} k \epsilon^{\prime}}{\beta}>1-\tau, \\
\left(1-\epsilon^{\prime}\right)^{k-3}>1-\tau, \\
\left(1+\epsilon^{\prime}\right)^{2}<1+\sigma .
\end{gathered}
$$

Set

$$
\epsilon=\min \left\{\epsilon_{13.5}, \frac{\epsilon_{13.2}}{2 \ell}, \epsilon_{13.10}, \frac{\epsilon_{10.3}}{4 \ell^{2}}, \epsilon^{\prime}\right\}
$$

This concludes the definitions of the constants.

Proof of Lemma 12.10. Let $k \geq 5$ be a given integer. Let $\alpha, \beta, \sigma, \gamma$ be given positive reals. Let $\delta$ be given in (78)-(79). Let $\ell>\frac{1}{\delta}$ be a given integer. Let $r$ and $\epsilon$ be given in (84) and (93) respectively. Let $n$ be sufficiently large, and suppose $\mathscr{H}$ is a $k$-partite 3-cylinder and $G=\bigcup_{1 \leq i<j \leq k} G^{i j}$ is an underlying cylinder which together satisfy the conditions of Setup 1 with constants $\alpha, \delta, \ell, r, \epsilon$, and $n$ and 3-uniform hypergraph $\mathscr{F}_{0}=$ $K_{k}^{(3)}$. We show that, for all but less than $\beta n$ good vertices $v \in V_{1}$, the number of link edges of $v$ which are $\sigma$-sparse with respect to $v$ is less than $\gamma \frac{\alpha}{\ell}\left(\frac{n}{\ell}(1+\epsilon)\right)^{2}$.

On the contrary, let $D$ be a set of good vertices $v \in V_{1}$ (cf. Definition 12.8), where $|D|$ $=\beta n$, such that, for each $v \in D$, there exists a set $E_{v}$ of link edges $e \in L_{v}^{23}$, each of which is $\sigma$-sparse with respect to $v$ (cf. Definition 12.9), where $\left|E_{v}\right|=\gamma \frac{\alpha}{\ell}\left(\frac{n}{\ell}(1+\epsilon)\right)^{2}$ (here for simplicity we omit integer part notation). We produce a contradiction under this assumption, hence proving Lemma 12.10 .

To that effect, we begin by defining the following set $\mathscr{Y} \subseteq \mathscr{K}_{k}(\mathscr{H})$ as

$$
\mathscr{Y}=\left\{Y \in \mathscr{K}_{k}(\mathscr{H}) \text { : for some } v \in D \text { and link edge } e=\{x, y\} \in E_{v},\{v, x, y\} \in[Y]^{3}\right\} \text {. }
$$

We estimate $|y|$ from above and below. To see the upper bound, it follows from our assumptions on the sizes $|D|$, and for each $v \in D,\left|E_{v}\right|$, and the definition of a link edge of $v$ which is $\sigma$-sparse with respect to $v$, that 


$$
\begin{aligned}
& |y| \leq \sum_{v \in D}\left|E_{v}\right| \frac{\alpha^{\left(\begin{array}{l}
k \\
3
\end{array}\right)-1}}{\ell^{\left(\begin{array}{l}
k \\
2
\end{array}\right)-3}} n^{k-3}(1-\sigma), \\
& =\beta n \gamma \frac{\alpha}{\ell}\left(\frac{n}{\ell}(1+\epsilon)\right)^{2} \frac{\alpha^{\left(\begin{array}{l}
k \\
3
\end{array}\right)-1}}{\ell^{\left(\begin{array}{l}
k \\
2
\end{array}\right)-3}} n^{k-3}(1-\sigma), \\
& =\beta \gamma(1-\sigma)(1+\epsilon)^{2} \frac{\alpha^{\left(\begin{array}{c}
k \\
3
\end{array}\right)}}{\ell\left(\begin{array}{c}
k \\
2
\end{array}\right)} n^{k}, \\
& \leq \beta \gamma(1-\sigma)(1+\sigma) \frac{\alpha^{\left(\begin{array}{l}
k \\
3
\end{array}\right)}}{\ell\left(\begin{array}{l}
k \\
2
\end{array}\right)} n^{k},
\end{aligned}
$$

where the inequality in (95) follows from our choice of $\epsilon$ in (92).

On the other hand, we now show that

$$
|y|>\beta \gamma\left(1-\sigma^{2}\right) \frac{\alpha^{\left(\begin{array}{l}
k \\
3
\end{array}\right)}}{\ell^{\left(\begin{array}{l}
k \\
2
\end{array}\right)}} n^{k},
$$

thus producing a contradiction with (95). The proof of (96) will be more complicated and its proof will only be completed after a series of claims.

To obtain the lower bound in (96), we begin our work by fixing $v \in D$. We first recall that $D$ is a set of good vertices, so according to Definition 12.8 , the fixed vertex $v \in D$ satisfies that, for all $i, j, 1<i<j \leq k,\left(L_{v}^{i j}, G^{i j}(v, G)\right)$ is an $\left(\frac{\alpha-2 \sqrt{\delta}}{\ell}, \sqrt{\delta}, \ell, r, 2 \ell \epsilon\right)$ regular couple. Consider the $(k-1)$-partite graphs $\bigcup_{1<i<j \leq k} L_{v}^{i j}$ and $\bigcup_{1<i<j \leq k}^{\ell} G^{i j}(v, G)$. It follows that these graphs satisfy the conditions of Setup 2 with the constants $k-1, \alpha$, $\sqrt{\delta}, \ell, r$, and $2 \epsilon \ell$, where again due to Definition 12.8 , for each $t, 1<t \leq k$, the size of each partite set $V_{t} \cap V\left(\bigcup_{1<i<j \leq k} G^{i j}(v, G)\right)=N_{G, t}(v)$ is at least $\frac{n}{\ell}(1-\epsilon)$. [In general, we use $V(J)$ to denote the vertex set of a graph $J$.] We intend to use Proposition 13.2 to conclude that as a result of $v \in D$ being a good vertex, almost all of the edges $e \in$ $E_{v}$ are good edges (with respect to suitable constants) (cf. Definition 13.3). We prove this fact precisely in the following claim. For what follows, recall that for $\alpha, \beta, \sigma$ and $\gamma$ given in Proposition 12.10, we defined auxiliary constants $\tau$ and $\beta^{\prime}$ in (70) and (71).

Claim 13.11. For the fixed vertex $v \in D$ and for the constants $\delta_{13.5}=\delta_{13.5}(\alpha, \tau), r_{13.5}=$ $r_{13.5}\left(\alpha, \tau, \delta_{13.5}, \ell\right)$, and $\epsilon_{13.5}=\epsilon_{13.5}\left(\alpha, \tau, \delta_{13.5}, \ell, r_{13.5}\right)$ in (72), (80) and (85) respectively, at least $\left(1-\frac{2 \beta^{\prime}}{\alpha \gamma}\right)\left|E_{v}\right|$ edges $e \in E_{v}$ are good link edges for the constants $\delta_{13.5}, r_{13.5}$, and $\epsilon_{13.5}$ (cf. Definition 13.3).

Proof of Claim 13.11. Claim 13.11 follows from the fact that since $v$ is a good vertex and due to our choices of $\delta, r$ and $\epsilon$ in (78), (84), and (93), we may apply Proposition 13.2 to the graphs $\bigcup_{1<i<j \leq k} L_{v}^{i j}$ and $\bigcup_{1<i<j \leq k} G^{i j}(v, G)$. Note that, in (78), $\sqrt{\delta} \leq \delta_{13.2}$, where $\delta_{13.2}=\delta_{13.2}\left(\alpha, \delta_{13.5}, \beta^{\prime}\right)$ is given in (73). Note that, in (84), $r \geq r_{13.2}$, where $r_{13.2}=r_{13.2}\left(\alpha, \delta_{13.5}, \beta^{\prime}, \delta_{13.2}, \ell, r_{13.5}\right)$ is given in (81). Note that in (93), $2 \ell \epsilon \leq \epsilon_{13.2}$, 
where $\epsilon_{13.2}=\epsilon_{13.2}\left(\alpha, \delta_{13.5}, \beta^{\prime}, \delta_{13.2}, \ell, r_{13.5}, r_{13.2}, \epsilon_{13.5}\right)$ is given in (86). Thus, by our choice of constants $\delta, r$ and $\epsilon$, we may apply Proposition 13.2 to the graphs $\bigcup_{1<i<j \leq k} L_{v}^{i j}$ and $\bigcup_{1<i<j \leq k} G^{i j}(v, G)$. Since the pair of graphs $\bigcup_{1<i<j \leq k} L_{v}^{i j}$ and $\bigcup_{1<i<j \leq k} G^{i j}(v, G)$ satisfies the hypothesis of Setup 2 with the constants $k-1, \alpha, \sqrt{\delta}$, $\ell, r$, and $2 \epsilon \ell$, we conclude from Proposition 13.2 that all but $\beta^{\prime}\left|L_{v}^{23}\right|$ edges $e \in L_{v}^{23}$ satisfy the conclusion of Claim 13.11. Note that $\left|L_{v}^{23}\right| \leq \frac{1}{\ell}(1+\epsilon)\left(\frac{n}{\ell}(1+\epsilon)\right)^{2} \leq 2 \frac{n^{2}}{\ell^{3}}$. Also, recall it was our assumption that $\left|E_{v}\right|=\gamma \frac{\alpha}{\ell}\left(\frac{n}{\ell}(1+\epsilon)\right)^{2} \geq \alpha \gamma \frac{n^{2}}{\ell^{3}}$. Consequently, $\left|L_{v}^{23}\right| \leq \frac{2}{\alpha \gamma}\left|E_{v}\right|$, and we thus conclude that all but $\frac{2 \beta^{\prime}}{\alpha \gamma}\left|E_{v}\right|$ edges $e \in E_{v}$ are good link edges with respect to the constants $\delta_{13.5}, r_{13.5}$ and $\epsilon_{13.5}$.

For $v \in D$ fixed, set $E_{v}^{\prime} \subseteq E_{v}$ to be the set of all link edges of $v$ which are good link edges with respect to the constants $\delta_{13.5}, r_{13.5}$ and $\epsilon_{13.5}$. Note that, by Claim 13.11,

$$
\left|E_{v}^{\prime}\right|>\left(1-\frac{2 \beta^{\prime}}{\alpha \gamma}\right)\left|E_{v}\right|
$$

Fix $e \in E_{v}^{\prime}$. We are interested in counting how many copies of $K_{k-3}^{(2)}$ are contained within the $(k-3)$-partite graph $\bigcup_{3<i<j \leq k} L_{v}^{i j}\left(e, L_{v}\right)$ (cf. (68)) (later, we will see that some of these underlie special copies of $K_{k-3}^{(3)}$ ). The following claim gives an estimate on this count.

\section{Claim 13.12.}

$$
\left|\mathcal{K}_{k-3}\left(\bigcup_{3<i<j \leq k} L_{v}^{i j}\left(e, L_{v}\right)\right)\right| \geq\left(\frac{\alpha}{\ell}\right)^{\left(\begin{array}{c}
k-3 \\
2
\end{array}\right)}\left(\left(\frac{\alpha-2 \sqrt{\delta}}{\ell}\right)^{2} \frac{n}{\ell}(1-\epsilon)\right)^{k-3}(1-\tau) .
$$

Proof of Claim 13.12. We note that Claim 13.12 essentially follows from the fact that due to our choice of constants, we may apply Fact 13.5 to the graph $\bigcup_{3<i<j \leq k} L_{v}^{i j}\left(e, L_{v}\right)$ together with the graph $\bigcup_{3<i<j \leq k} G_{v}^{i j}\left(e, L_{v}\right)$. Indeed, $e \in E_{v}^{\prime}$ is a good link edge with respect to the constants $\delta_{13.5}, r_{13.5}, \epsilon_{13.5}$, so, for each $i, j, 3<i<j \leq k,\left(L_{v}^{i j}\left(e, L_{v}\right)\right.$, $\left.G_{v}^{i j}\left(e, L_{v}\right)\right)$ is an $\left(\frac{\alpha-2 \delta_{13.5}}{\ell}, \delta_{13.5}, r_{13.5}, \ell, \epsilon_{13.5}\right)$-regular couple, where the constants $\delta_{13.5}=\delta_{13.5}(\alpha, \tau), r_{13.5}=r_{13.5}\left(\alpha, \tau, \delta_{13.5}, \ell\right)$, and $\epsilon_{13.5}=\epsilon_{13.5}\left(\alpha, \tau, \delta_{13.5}, \ell, r_{13.5}\right)$ [cf. (72), (80), and (85)]. Thus, the pair of $(k-3)$-partite graphs $\bigcup_{3<i<j \leq k} L_{v}^{i j}\left(e, L_{v}\right)$ and $\bigcup_{3<i<j \leq k} G_{v}^{i j}\left(e, L_{v}\right)$ satisfy the conditions of Setup 2 with the constants $k-3, \alpha, \delta_{13.5}$, $\ell, r_{13.5}$, and $\epsilon_{13.5}$. With $\delta_{13.5}, r_{13.5}$, and $\epsilon_{13.5}$ appropriately given, it thus follows that Fact 13.5 applies to the pair of graphs $\bigcup_{3<i<j \leq k} L_{v}^{i j}\left(e, L_{v}\right)$ and $\bigcup_{3<i<j \leq k} G_{v}^{i j}\left(e, L_{v}\right)$ with the constants $\alpha, \tau, \delta_{13.5}, \ell, r_{13.5}$, and $\epsilon_{13.5}$. It is essentially this application that renders the statement of Claim 13.12. However, to complete the application, we also need to know the sizes $\left|V\left(\bigcup_{3<i<j \leq k} L_{v}^{i j}\left(e, L_{v}\right)\right) \cap V_{t}\right|=\left|N_{L_{v}, t}(e)\right|, 3<t \leq k$, of each partite set of $\bigcup_{3<i<j \leq k} L_{v}^{i j}\left(e, L_{v}\right)$. However, according to Definition 13.3, for each $t, 3<t \leq k$,

$$
\left|V\left(\bigcup_{3<i<j \leq k} L_{v}^{i j}\left(e, L_{v}\right)\right) \cap V_{t}\right|=\left|N_{L_{v}, t}(e)\right|>\left(\frac{\alpha-2 \sqrt{\delta}}{\ell}\right)^{2} \frac{n}{\ell}(1-\epsilon) .
$$


We therefore see from Fact 13.5 that

$$
\left|\mathscr{K}_{k-3}\left(\bigcup_{3<i<j \leq k} L_{v}^{i j}\left(e, L_{v}\right)\right)\right|>\left(\frac{\alpha}{\ell}\right)^{\left(\begin{array}{c}
k-3 \\
2
\end{array}\right)}\left(\left(\frac{\alpha-2 \sqrt{\delta}}{\ell}\right)^{2} \frac{n}{\ell}(1-\epsilon)\right)^{k-3}(1-\tau) .
$$

Thus, our proof of Claim 13.12 is complete.

We now prepare to use Claim 13.12. For $v \in D$ and $e \in E_{v}^{\prime}$ fixed above, let $e=\{x$, $y$ \}. Define

$$
\begin{aligned}
& A_{x}^{(v)}=\left\{\{x, a\} \in L_{v}: a \in V\left(\bigcup_{3<i<j \leq k} L_{v}^{i j}\left(e, L_{v}\right)\right)\right\}, \\
& B_{y}^{(v)}=\left\{\{y, b\} \in L_{v}: b \in V\left(\bigcup_{3<i<j \leq k} L_{v}^{i j}\left(e, L_{v}\right)\right)\right\} .
\end{aligned}
$$

Define

$$
C_{e}^{(v)}=C_{\{x, y\}}^{(v)}=\{x, y\} \cup A_{x}^{(v)} \cup B_{y}^{(v)} \cup \underset{3<i<j \leq k}{\bigcup} L_{v}^{i j}\left(e, L_{v}\right)
$$

Note that $C_{e}^{(v)}$ is a $(k-1)$-partite graph on vertex sets $N_{G, 2}(v), \ldots, N_{G, k}(v)$. Note also that

$$
\left|\mathscr{K}_{k-1}\left(C_{e}^{(v)}\right)\right|=\left|\mathscr{K}_{k-3}\left(\bigcup_{3<i<j \leq k} L_{v}^{i j}\left(e, L_{v}\right)\right)\right|
$$

and that

$$
\bigcup_{e \in E_{v}^{\prime}} \mathscr{K}_{k-1}\left(C_{e}^{(v)}\right)=\mathscr{K}_{k-1}\left(\bigcup_{e \in E_{v}^{\prime}} C_{e}^{(v)}\right)
$$

For $v \in D$ fixed, we use Claim 13.12 to compute a lower bound on $\left|\bigcup_{e \in E_{v}^{\prime}} \mathcal{K}_{k-1}\left(C_{e}^{(v)}\right)\right|$, and after providing this lower bound, we pause to reveal our strategy for proving (96).

As $\bigcup_{e \in E_{\nu}^{\prime}} \mathcal{K}_{k-1}\left(C_{e}^{(v)}\right)$ is a disjoint union, we see by Claim 13.12, (98), and the fact that $\left|E_{v}^{\prime}\right| \geq\left(1-\frac{2 \beta^{\prime}}{\alpha \gamma}\right) \gamma \frac{\alpha}{\ell}\left(\frac{n}{\ell}(1+\epsilon)\right)^{2}$ from (97) that $\left|\bigcup_{e \in E_{v}^{\prime}} \mathscr{K}_{k-1}\left(C_{e}^{(v)}\right)\right|$ has lower bound

$$
\left(\frac{\alpha}{\ell}\right)^{\left(\begin{array}{c}
k-1 \\
2
\end{array}\right)-1}\left(\frac{n}{\ell}\right)^{k-3}\left(1-\frac{2 \sqrt{\delta}}{\alpha}\right)^{2(k-3)}(1-\epsilon)^{k-3}(1-\tau)\left(1-\frac{2 \beta^{\prime}}{\alpha \gamma}\right) \gamma \frac{\alpha}{\ell}\left(\frac{n}{\ell}(1+\epsilon)\right)^{2} \text {. }
$$

As a result of (71), (79), and (91), we conclude that (100) can be estimated from below to yield 


$$
\left|\bigcup_{e \in E_{v}^{\prime}} \mathcal{K}_{k-1}\left(C_{e}^{(v)}\right)\right|>\gamma\left(\frac{\alpha}{\ell}\right)^{\left(\begin{array}{c}
k-1 \\
2
\end{array}\right)}\left(\frac{n}{\ell}\right)^{k-1}(1-\tau)^{4} .
$$

We use (101) to prove (96). Our goal is to take a set of $r_{k-1}$ [cf. (82)] vertices $v \in D$, and use their graphs $\bigcup_{e \in E_{v}^{\prime}} C_{e}^{(v)}$ to build an $r_{k-1}$-tuple of $(k-1)$-partite cylinders [an $r_{k-1}$-tuple of $(k-1)$-partite cylinders in the sense of Definition 13.9]. We then wish to invoke Lemma 13.10 to infer that many of the copies of $K_{k-1}^{(2)}$ in $\bigcup_{e \in E_{v}^{\prime}} C_{e}^{(v)}$, over all $r_{k-1}$ vertices $v \in D$ specially chosen, must underlie copies of $K_{k-1}^{(3)}$ in $\mathscr{H}$. We make our plan precise in what follows, and begin by using Fact 13.6 given earlier to select the special set of $r_{k-1}$ vertices $v \in D$.

We define the following graph $G_{D}$ to which we apply Fact 13.6. For $1=i<j \leq k$, set $G_{D}^{i j}=G_{D}^{1 j}$ to be the subgraph of $G^{1 j}$ induced on the sets $D$ and $V_{j}$, that is,

$$
G_{D}^{i j}=G_{D}^{1 j}=G\left[D, V_{j}\right] .
$$

For $1<i<j \leq k$, set

$$
G_{D}^{i j}=G^{i j}
$$

and

$$
G_{D}=\bigcup_{1 \leq i<j \leq k} G_{D}^{i j}
$$

Recalling the notation from Fact 13.6, set $W_{0}=D$, and for $1 \leq i \leq k-1, W_{i}=V_{i+1}$, and set $r$ from the hypothesis of Fact 13.6 to be equal to $r_{k-1}$ [cf. (82)]. Note that the fact that $G$ is an $(\ell, \epsilon, k)$-cylinder implies that Condition (i) in the hypothesis of Fact 13.6 is satisfied. Since every vertex $v \in D$ satisfies $\left|N_{G_{D}, i}(v)\right|=\left|N_{G, i}(v)\right| \leq \frac{n}{\ell}(1+\epsilon)$, Condition (ii) in the hypothesis of Fact 13.6 is satisfied. Therefore, applying Fact 13.6 to the graph $G_{D}$ with the constants $k, r_{k-1}, \ell, \epsilon$, and $\beta$ implies that there exists a collection $\mathscr{D}=\left\{D_{1}, \ldots, D_{q}\right\}$ of pairwise disjoint $r_{k-1}$-element subsets of $D$ satisfying the conclusion of Fact 13.6. We recall here that $q=\left\lceil\frac{\beta n}{r_{k-1}}\left(1-r_{k-1} k \epsilon / \beta\right)\right\rceil$. We use the notation

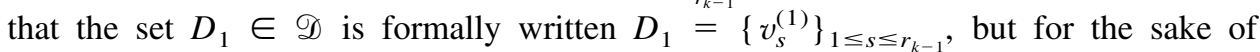
convenience, we often use the notation that the elements of $D_{1}$ are simply written as $v_{s}$, $1 \leq s \leq r_{k-1}$.

Define the $r_{k-1}$-tuple $\vec{Q}_{1}$ by

$$
\vec{Q}_{1}=\left(\bigcup_{e \in E_{v_{s}}^{\prime}} C_{e}^{\left(v_{s}\right)}\right)_{v_{s} \in D_{1}}
$$

We proceed with the following claim.

Claim 13.13. With $\vec{Q}_{1}$ defined in (102) and $\delta_{k-1}$ given in (74) and (76),

$$
\left|\bigcup_{v_{s} \in D_{1}} \mathscr{K}_{k-1}\left(\bigcup_{e \in E_{v_{s}}^{\prime}} C_{e}^{\left(v_{s}\right)}\right)\right|>\delta_{k-1}\left|\mathscr{K}_{k-1}(G(\{2, \ldots, k\}))\right| .
$$


Proof of Claim 13.13. We obtain through Inclusion-Exclusion that

$$
\begin{gathered}
\left|\bigcup_{v_{s} \in D_{1}} \mathscr{K}_{k-1}\left(\bigcup_{e \in E_{v_{s}}^{\prime}} C_{e}^{\left(v_{s}\right)}\right)\right| \geq \sum_{v_{s} \in D_{1}}\left|\mathscr{K}_{k-1}\left(\bigcup_{e \in E_{v_{s}}^{\prime}} C_{e}^{\left(v_{s}\right)}\right)\right| \\
-\sum_{\left\{v_{s}, v_{s}^{\prime}\right\} \in\left[D_{1}\right]^{2}}\left|\mathscr{K}_{k-1}\left(\bigcup_{e \in E_{v_{s}}^{\prime}} C_{e}^{\left(v_{s}\right)}\right) \cap \mathscr{K}_{k-1}\left(\bigcup_{e \in E_{v_{s}^{\prime}}^{\prime}} C_{e}^{\left(v_{s}^{\prime}\right)}\right)\right| .
\end{gathered}
$$

To bound the second-order term, note that it follows that, for each $\left\{v_{s}, v_{s}^{\prime}\right\} \in\left[D_{1}\right]^{2}$,

$$
\left|\mathscr{K}_{k-1}\left(\bigcup_{e \in E_{v_{s}}^{\prime}} C_{e}^{\left(v_{s}\right)}\right) \cap \mathcal{K}_{k-1}\left(\bigcup_{e \in E_{v_{s}^{\prime}}^{\prime}} C_{e}^{\left(v_{s}^{\prime}\right)}\right)\right| \leq\left|\mathscr{K}_{k-1}\left(\bigcup_{1<i<j \leq k}\left(G^{i j}\left(v_{s}, G\right) \cap\left(G^{i j}\left(v_{s}^{\prime}, G\right)\right)\right)\right)\right| .
$$

It follows from property $(b)$ of Fact 13.6 and Fact 10.3 [cf. $\epsilon$ given in (93)] that

$$
\mid \mathscr{K}_{k-1}\left(\bigcup_{1<i<j \leq k}\left(G^{i j}\left(v_{s}, G\right) \cap\left(G^{i j}\left(v_{s}^{\prime}, G\right)\right)\right) \mid<2\left(\frac{1}{\ell}(1+\epsilon)\right)^{\left(\begin{array}{c}
k-1 \\
2
\end{array}\right)}\left[\frac{n}{\ell^{2}}(1+\epsilon)^{2}\right]^{k-1} .\right.
$$

Combining our bounds on the second-order term with the equality in (99) and the fact that $\left|D_{1}\right|=r_{k-1}$, we conclude that

$$
\begin{aligned}
& \left|\bigcup_{v_{s} \in D_{1}} \mathscr{K}_{k-1}\left(\bigcup_{e \in E_{v_{s}}^{\prime}} C_{e}^{\left(v_{s}\right)}\right)\right|>\sum_{v_{s} \in D_{1}}\left|\bigcup_{e \in E_{v_{s}}^{\prime}} \mathcal{K}_{k-1}\left(C_{e}^{\left(v_{s}\right)}\right)\right| \\
& -\left(\begin{array}{c}
r_{k-1} \\
2
\end{array}\right) 2\left(\frac{1}{\ell}(1+\epsilon)\right)^{\left(\begin{array}{c}
k-1 \\
2
\end{array}\right)}\left[\frac{n}{\ell^{2}}(1+\epsilon)^{2}\right]^{k-1} .
\end{aligned}
$$

Using (101) and again that $\left|D_{1}\right|=r_{k-1}$, we further conclude that the quantity above has lower bound

$$
r_{k-1} \gamma\left(\frac{\alpha}{\ell}\right)^{\left(\begin{array}{c}
k-1 \\
2
\end{array}\right)}(1-\tau)^{4}\left(\frac{n}{\ell}\right)^{k-1}-\left(\begin{array}{c}
r_{k-1} \\
2
\end{array}\right) 2\left(\frac{1}{\ell}(1+\epsilon)\right)^{\left(\begin{array}{c}
k-1 \\
2
\end{array}\right)}\left[\frac{n}{\ell^{2}}(1+\epsilon)^{2}\right]^{k-1}
$$

which in turn has lower bound

$$
\left(\frac{\alpha}{\ell}\right)^{\left(\begin{array}{c}
k-1 \\
2
\end{array}\right)} n^{k-1}\left[r_{k-1} \frac{\gamma(1-\tau)^{4}}{\ell^{k-1}}-r_{k-1}^{2} \frac{(1+\epsilon)\left(\begin{array}{c}
k+1 \\
2
\end{array}\right)-1}{\alpha\left(\begin{array}{c}
k-1 \\
2
\end{array}\right) \ell^{2(k-1)}}\right]
$$

Substituting the value $r_{k-1}=\zeta \ell^{k-1}$ [cf. (82)] into (103) yields 


$$
\begin{gathered}
\left(\frac{\alpha}{\ell}\right)^{\left(\begin{array}{c}
k-1 \\
2
\end{array}\right)} n^{k-1} \zeta\left[\gamma(1-\tau)^{4}-\frac{\zeta}{\alpha\left(\begin{array}{c}
k-1 \\
2
\end{array}\right)}(1+\epsilon)\left(\begin{array}{c}
k+1 \\
2
\end{array}\right)-1\right] \\
>\left(\frac{\alpha}{\ell}\right)^{\left(\begin{array}{c}
k-1 \\
2
\end{array}\right)} n^{k-1} \zeta\left[\frac{\gamma}{2}-\frac{2 \zeta}{\alpha\left(\begin{array}{c}
k-1 \\
2
\end{array}\right)}\right]
\end{gathered}
$$

where the last inequality follows from (70) and (89). Since $\zeta<\frac{\gamma}{8} \alpha\left(\begin{array}{c}k-1 \\ 2\end{array}\right)$ in (74), we see that

$$
\frac{\gamma}{2}-\frac{2 \zeta}{\alpha\left(\begin{array}{c}
k-1 \\
2
\end{array}\right)}>\frac{\gamma}{4}
$$

Since $\zeta=\frac{8 \delta_{k-1}}{\alpha\left(\begin{array}{c}k-1 \\ 2\end{array}\right) \gamma}$, we have that (104) is bounded from below by

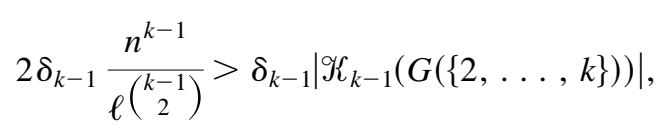

where the last inequality follows from Fact 10.3 [cf. $\epsilon$ given in (93)]. We therefore conclude that

$$
\left|\bigcup_{v_{s} \in D_{1}} \mathcal{K}_{k-1}\left(\bigcup_{e \in E_{v_{s}}^{\prime}} C_{e}^{\left(v_{s}\right)}\right)\right|>\delta_{k-1}\left|\mathscr{K}_{k-1}(G(\{2, \ldots, k\}))\right|,
$$

and hence the proof of Claim 13.13 is complete.

We now prepare to use Claim 13.13. Not that since $\mathcal{H}$ and $G=\bigcup_{1 \leq i<j \leq k} G^{i j}$ satisfy the conditions of Setup 1 with the constants $k, \alpha, \delta, \ell, r, \epsilon$, and $n$ and 3-uniform hypergraph $\mathscr{F}_{0}=K_{k}^{(3)}$, it trivially follows that $\mathscr{H}(\{2, \ldots, k\})$ and $G(\{2, \ldots, k\})$ satisfy the conditions of Setup 1 with the constants $k-1, \alpha, \delta, \ell, r, \epsilon$, and $n$ and 3-uniform hypergraph $\mathscr{g}_{0}=K_{k-1}^{(3)}$. We wish to invoke Lemma 13.10 to conclude that $\mathscr{H}(\{2, \ldots, k\})$ is $\left(\alpha\left(\begin{array}{c}k-1 \\ 3\end{array}\right), \delta_{k-1}, r_{k-1}\right)$-regular with respect to $G(\{2, \ldots, k\})$. However, the $\left(\alpha\left(\begin{array}{c}k-1 \\ 3\end{array}\right), \delta_{k-1}, r_{k-1}\right)$-regularity of $\mathscr{H}(\{2, \ldots, k\})$ with respect to $G(\{2, \ldots$, $k\}$ ) is guaranteed by our choice of constants $\delta, r$, and $\epsilon$. Indeed, recall that our choice of constants $\delta, r$, and $\epsilon$ satisfied $\delta \leq \delta_{13.10}\left(\alpha, \delta_{k-1}\right)$ in (77), $r \geq r_{13.10}\left(\alpha, \delta_{k-1}, \delta_{13.10}\right.$, $\left.\ell, r_{k-1}\right)$ in (83), and $\epsilon \leq \epsilon_{13.10}\left(\alpha, \delta_{k-1}, \delta_{13.10}, \ell, r_{k-1}, r_{13.10}\right)$ in (87), respectively. Since $\mathscr{H}(\{2, \ldots, k\})$ and $G(\{2, \ldots, k\})$ satisfy the hypothesis of Setup 1 with constants $k-1, \alpha, \delta, \ell, r, \epsilon$, and 3-uniform hypergraph $\mathscr{F}_{0}=K_{k-1}^{(3)}$, it follows from Lemma 13.10 that the $(k-1)$-partite 3 -cylinder $\mathscr{H}(\{2, \ldots, k\})$ is $\left(\alpha\left(\begin{array}{c}k-1 \\ 3\end{array}\right), \delta_{k-1}, r_{k-1}\right)$-regular with respect to $G(\{2, \ldots, k\})$.

Now we may infer that an $\left.\alpha{ }^{(k-1} 3\right)$ portion of the cliques $K_{k-1}^{(2)}$ counted in Claim 13.13 underlie cliques $K_{k-1}^{(3)}$ in $\mathscr{H}(\{2, \ldots, k\})$. More precisely, we conclude from Claim 13.13 and the $\left(\alpha\left(\begin{array}{c}k-1 \\ 3\end{array}\right), \delta_{k-1}, r_{k-1}\right)$-regularity of $\mathcal{H}(\{2, \ldots, k\})$ with respect to $G(\{2, \ldots, k\})$ that 


$$
\frac{\left|\mathscr{K}_{k-1}(\mathcal{H}(\{2, \ldots, k\})) \cap \bigcup_{v_{s} \in D_{1}} \mathscr{K}_{k-1}\left(\bigcup_{e \in E_{v_{s}}^{\prime}} C_{e}^{\left(v_{s}\right)}\right)\right|}{\left|\bigcup_{v_{s} \in D_{1}} \mathscr{K}_{k-1}\left(\bigcup_{e \in E_{v_{s}}^{\prime}} C_{e}^{\left(v_{s}\right)}\right)\right|}>\alpha\left(\begin{array}{c}
k-1 \\
3
\end{array}\right)-2 \delta_{k-1}
$$

By the left-hand side lower estimate of $\left|\bigcup_{v_{s} \in D_{1}} \mathcal{K}_{k-1}\left(\bigcup_{e \in E_{v_{s}}^{\prime}} C_{e}^{\left(v_{s}\right)}\right)\right|$ in (104), we infer from the inequality above that

$$
\begin{aligned}
& \left|\mathscr{K}_{k-1}(\mathscr{H}(\{2, \ldots, k\})) \cap \bigcup_{v_{s} \in D_{1}} \mathscr{K}_{k-1}\left(\bigcup_{e \in E_{v_{s}}^{\prime}} C_{e}^{\left(v_{s}\right)}\right)\right| \\
& >\left(\alpha\left(\begin{array}{c}
k-1 \\
3
\end{array}\right)-2 \delta_{k-1}\right)\left(\frac{\alpha}{\ell}\right) \stackrel{\left(\begin{array}{c}
k-1 \\
2
\end{array}\right)}{n^{k-1} \zeta}\left[\gamma(1-\tau)^{4}-\frac{\zeta}{\alpha\left(\begin{array}{c}
k-1 \\
2
\end{array}\right)}(1+\epsilon)\left(\begin{array}{c}
k+1 \\
2
\end{array}\right)-1\right] .
\end{aligned}
$$

We now use (107) to prove (96), and prepare its use below. First, recall the set $y$ defined in (94). For $v \in D$ fixed, define

$$
\mathscr{Y}_{v}=\{Y \in \mathscr{Y}: v \in Y\} .
$$

Note that, for each $v, v^{\prime} \in D, v \neq v^{\prime}, \mathscr{Y}_{v} \cap \mathscr{Y}_{v^{\prime}}=\varnothing$. Also note that

$$
y=\bigcup_{v \in D} y_{v} \text {. }
$$

Now recall the collection $\mathscr{D}=\left\{D_{1}, \ldots, D_{q}\right\}$ of pairwise disjoint subsets of $D$ obtained by the application of Fact 13.6, where we recall $q=\left\lceil\frac{\beta n}{r_{k-1}}\left(1-r_{k-1} k \epsilon / \beta\right)\right\rceil$. With $\bigcup_{t=1}^{q} D_{t} \subseteq D$, we conclude that from (109) that

$$
y \supseteq \bigcup_{t=1}^{q} \bigcup_{v \in D_{t}} y_{v} .
$$

By the pairwise disjointness of the $y_{v}$, s, we further conclude

$$
|\mathscr{Y}| \geq \sum_{t=1}^{q}\left|\bigcup_{v \in D_{t}} \mathscr{Y}_{v}\right|
$$

We now fix $t \in[q]$ and consider the quantity $\left|\bigcup_{v \in D_{t}} \mathscr{Y}_{v}\right|$. To that end, for $v \in D_{t}$ fixed, define the following set $y_{v}^{-}$related to (108) as

$$
\mathscr{Y}_{v}^{-}=\left\{Y \backslash\{v\}: \mathscr{y} \in \mathscr{Y}_{v}\right\},
$$

and note that

$$
\left|y_{v}\right|=\left|y_{v}^{-}\right| .
$$

It follows that 


$$
\left|\bigcup_{v \in D_{t}} y_{v}\right| \geq\left|\bigcup_{v \in D_{t}} y_{v}^{-}\right|
$$

Now, for $v \in D_{t}$ fixed, it is not hard to see that

$$
\mathscr{Y}_{v}^{-}=\mathscr{K}_{k-1}(\mathscr{H}(\{2, \ldots, k\})) \cap \mathscr{K}_{k-1}\left(\bigcup_{e \in E_{v}} C_{e}^{(v)}\right)
$$

With $E_{v}^{\prime} \subseteq E_{v}$, where $\left|E_{v}^{\prime}\right|>\left(1-\frac{2 \beta^{\prime}}{\alpha \gamma}\right)\left|E_{v}\right|$ from (97), it follows from (112) that

$$
\mathscr{Y}_{v}^{-} \supseteq \mathscr{K}_{k-1}(\mathscr{H}(\{2, \ldots, k\})) \cup \mathscr{K}_{k-1}\left(\bigcup_{e \in E_{v}^{\prime}} C_{e}^{(v)}\right)
$$

It follows that

$$
\begin{aligned}
\bigcup_{v \in D_{t}} \mathscr{Y}_{v}^{-} & \supseteq \bigcup_{v \in D_{t}}\left(\mathscr{K}_{k-1}(\mathscr{H}(\{2, \ldots, k\})) \cap \mathscr{K}_{k-1}\left(\bigcup_{e \in E_{v}^{\prime}} C_{e}^{(v)}\right)\right), \\
& =\mathscr{K}_{k-1}(\mathscr{H}(\{2, \ldots, k\})) \cap \bigcup_{v \in D_{t}} \mathscr{K}_{k-1}\left(\bigcup_{e \in E_{v}^{\prime}} C_{e}^{(v)}\right),
\end{aligned}
$$

and so

$$
\left|\bigcup_{v \in D_{t}} \mathscr{Y}_{v}^{-}\right| \geq\left|\mathscr{K}_{k-1}(\mathscr{H}(\{2, \ldots, k\})) \cap \bigcup_{v \in D_{t}} \mathscr{K}_{k-1}\left(\bigcup_{e \in E_{v}^{\prime}} C_{e}^{(v)}\right)\right|
$$

Now by (107), we infer from (113) that

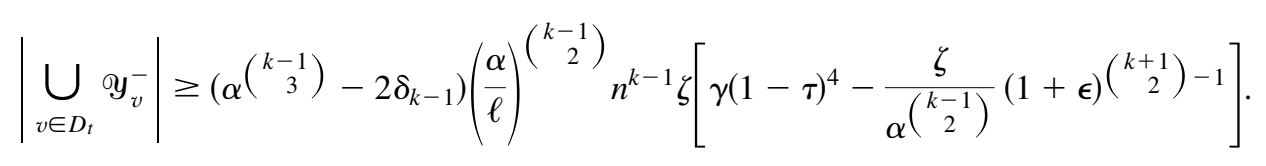

Set

$$
\Phi=\left[\gamma(1-\tau)^{4}-\frac{\zeta}{\alpha\left(\begin{array}{c}
k-1 \\
2
\end{array}\right)}(1+\epsilon)\left(\begin{array}{c}
k+1 \\
2
\end{array}\right)-1\right] .
$$

Thus, from (110) and the fact that $\left.q=\left\lceil\frac{\beta n}{r_{k-1}} 1-r_{k-1} k \epsilon / \beta\right)\right\rceil$, we obtain

$$
|y|>\frac{\beta n}{r_{k-1}}\left(1-\frac{r_{k-1} k \epsilon}{\beta}\right)\left(\alpha\left(\begin{array}{c}
k-1 \\
3
\end{array}\right)-2 \delta_{k-1}\right)\left(\frac{\alpha}{\ell}\right)^{\left(\begin{array}{c}
k-1 \\
2
\end{array}\right)} n^{k-1} \zeta \Phi .
$$

With $r_{k-1}=\zeta \ell^{k-1}$ from (82), we see the above quantity is equal to 


$$
\beta \gamma \frac{\alpha^{\left(\begin{array}{c}
k \\
3
\end{array}\right)}}{\ell^{\left(\begin{array}{c}
k \\
2
\end{array}\right)}} n^{k}\left(1-\frac{r_{k-1} k \epsilon}{\beta}\right)\left(1-\frac{2 \delta_{k-1}}{\alpha\left(\begin{array}{c}
k-1 \\
3
\end{array}\right)}\right)\left((1-\tau)^{4}-\frac{\zeta}{\gamma \alpha\left(\begin{array}{c}
k-1 \\
2
\end{array}\right)}(1+\epsilon)\left(\begin{array}{c}
k+1 \\
2
\end{array}\right)-1\right)
$$

With the inequalities in (75), (89), and (90), we see the above quantity has lower bound

$$
\beta \gamma \frac{\alpha\left(\begin{array}{l}
k \\
3
\end{array}\right)}{\ell\left(\begin{array}{l}
k \\
2
\end{array}\right)} n^{k}(1-\tau)^{2}\left((1-\tau)^{4}-\frac{2 \zeta}{\gamma \alpha\left(\begin{array}{c}
k-1 \\
2
\end{array}\right)}\right)
$$

Note that as a result of (76), $(1-\tau)^{4}-\frac{2 \zeta}{\gamma \alpha\left(\begin{array}{c}k-1 \\ 2\end{array}\right)}>(1-\tau)^{5}$. Thus together with (70), the quantity above may be bounded from below by

$$
\beta \gamma \frac{\alpha^{\left(\begin{array}{l}
k \\
3
\end{array}\right)}}{\ell^{\left(\begin{array}{l}
k \\
2
\end{array}\right)}} n^{k}(1-\tau)^{7} \geq \beta \gamma \frac{\alpha^{\left(\begin{array}{l}
k \\
3
\end{array}\right)}}{\ell^{\left(\begin{array}{l}
k \\
2
\end{array}\right)}} n^{k}\left(1-\sigma^{2}\right),
$$

and we thus conclude

$$
|\mathscr{y}|>\beta \gamma \frac{\alpha^{\left(\begin{array}{c}
k \\
3
\end{array}\right)}}{\ell^{\left(\begin{array}{c}
k \\
2
\end{array}\right)}} n^{k}\left(1-\sigma^{2}\right)
$$

Hence, (96) is established and Lemma 12.10 is proved.

\section{REFERENCES}

[1] S. Arora, D. Karger, and M. Karpinski, Polynomial time approximation schemes for dense instances of NP-hard problems, Proc ACM Symp Theory of Computing, Las Vegas, NV, 1995, pp. 284-293.

[2] A. Czygrinow, S. Poljak, and V. Rödl, Constructive quasi-Ramsey numbers and tournament ranking, SIAM J Discrete Mathematics 12 (1999), 48-63.

[3] D. Dor and M. Tarsi, Graph decomposition is NPC - a complete proof of Holyer's conjecture, Proc ACM Symp Theory of Computing, Victoria, BC, 1992, pp. 252-263.

[4] R. Duke, H. Lefmann, and V. Rödl, A fast algorithm for computing frequencies in a given graph, SIAM J Comput 24 (1995), 598-620.

[5] P. Frankl and V. Rödl, Near perfect coverings in graphs and hypergraphs, Eur J Combinat 6 (1985), 317-326.

[6] P. Frankl and V. Rödl, Extremal problems on set systems, Random Struct Alg 20 (2002), 131-164.

[7] A. Frieze and R. Kannan, The Regularity Lemma and approximation schemes for dense problems, Proc IEEE Symp Foundations of Computer Science, Burlington, VT, 1996, pp. 12-20.

[8] A. Frieze and R. Kannan, Quick approximation to matrices and applications, Combinatorica 19 (1999), 175-220.

[9] P. Haxell and V. Rödl, Integer and fractional packings in dense graphs, Combinatorica 21 (2001), 13-38.

[10] S. Janson, T. Łuczak, and A. Ruciński, Random graphs, Wiley, New York, 2000. 
[11] J. Kahn, A linear programming perspective on the Frankl-Rödl-Pippenger Theorem, Random Struct Alg 8 (1996), 149-157.

[12] B. Nagle, Regularity properties for triple systems, Ph.D. Thesis, Emory University, Atlanta, GA, 1999.

[13] B. Nagle and V. Rödl, Regularity properties for triple systems, Random Struct Alg, to appear.

[14] E. Szemerédi, "Regular partitions of graphs," Problèmes en combinatoire et théorie des Graphes, Proc Coll Int CNRS, J.-C. Bermond, J.-C. Fournier, M. Las Vergnas, and D. Sotteau, 1978, pp. 399-401. 